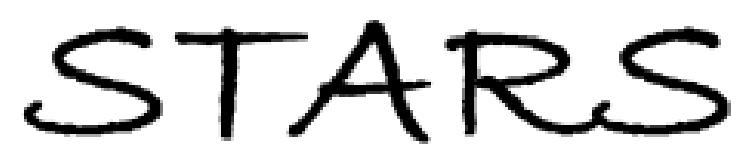

University of Central Florida

STARS

Electronic Theses and Dissertations, 2004-2019

2004

\title{
Modified Sierpinski Fractal Antenna
}

Tripti Luintel

University of Central Florida

Part of the Electrical and Electronics Commons

Find similar works at: https://stars.library.ucf.edu/etd

University of Central Florida Libraries http://library.ucf.edu

This Masters Thesis (Open Access) is brought to you for free and open access by STARS. It has been accepted for inclusion in Electronic Theses and Dissertations, 2004-2019 by an authorized administrator of STARS. For more information, please contact STARS@ucf.edu.

\section{STARS Citation}

Luintel, Tripti, "Modified Sierpinski Fractal Antenna" (2004). Electronic Theses and Dissertations, 2004-2019. 209.

https://stars.library.ucf.edu/etd/209

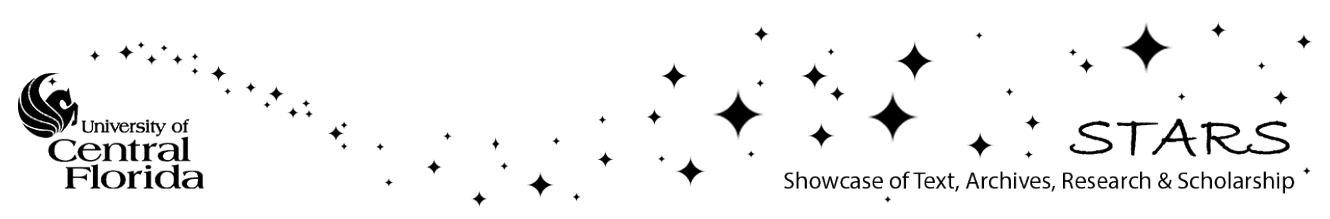




\section{MODIFIED SIERPINSKI FRACTAL ANTENNA}

by

TRIPTI LUINTEL

B.E. Kathmandu University, 2001

A thesis submitted in partial fulfillment of the requirements for the degree of Master of Science

in the Department of Electrical and Computer Engineering in the College of Engineering and Computer Science at the University of Central Florida

Orlando, Florida

Fall Term

2004 
(C) 2004 Tripti Luintel 


\begin{abstract}
Fractal antennas have the potential to provide multi-band solution through the property of self similarity that the fractal shape possess. This thesis deals with some modifications of the original Sierpinski fractal patch antenna, providing additional parameters to optimize in order to get the required radiation characteristics. A systematic study of the original Sierpinski monopole was done to gain an insight on the operation and multi-band behavior of the antenna and a similar study was done to find out why the patch version of Sierpinski lacks multi-band behavior. A Sierpinski antenna is designed by merging a grid and the fractal restoring the multi-band behavior. A stacked Sierpinski antenna is then designed by introducing an intermediate layer containing the triangular patch whose size is equal to that of the triangular hole in the fractal structure. The introduction of a gap in this structure improves the overall radiation pattern. The antennas are analyzed using the commercial IE3D package and the simulated radiation patterns are presented.
\end{abstract}




\section{ACKNOWLEDGMENTS}

I would like to express my gratitude to my advisor Dr. Parveen Wahid, for her constant support, guidance and valuable advice for the completion of this work and my graduate studies.

I am very grateful to Dr. Bernard C. Deloach Jr for his valuable ideas and expertise and to introduce me to researches in antenna.

I thank my colleagues who have supported and helped me with my studies. 


\section{TALBE OF CONTENTS}

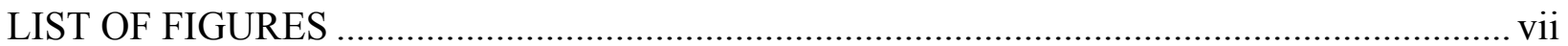

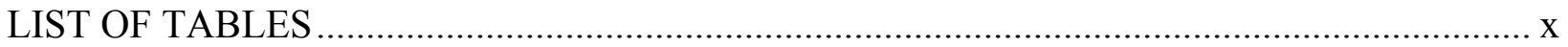

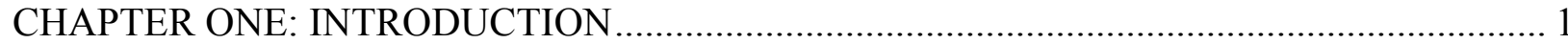

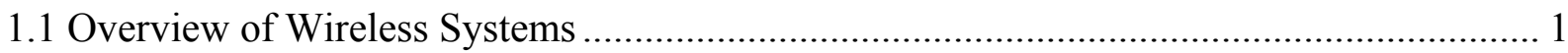

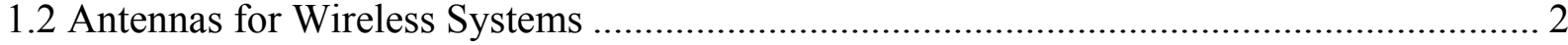

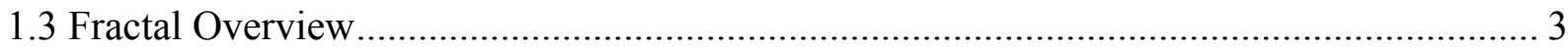

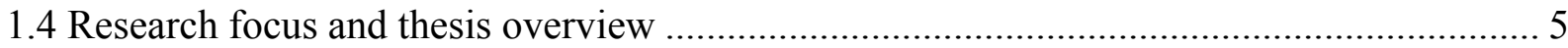

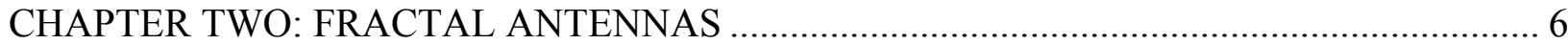

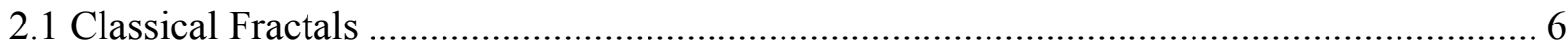

2.2 Iterated Function System …………………………......................................................

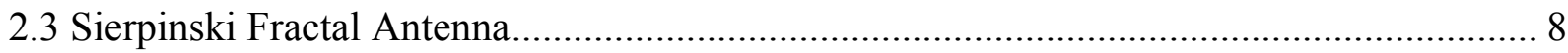

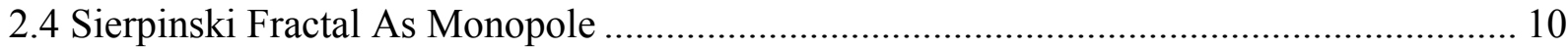

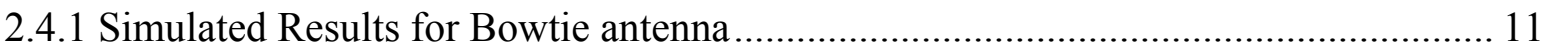

2.4.2 Simulated results for the Sierpinski monopole antenna................................................ 17

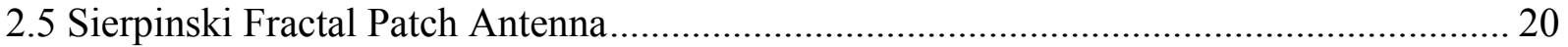

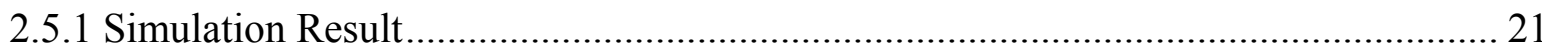

CHAPTER THREE: STACKED FRACTAL ANTENNA DESIGN ……………………............. 31

3.1 Recent Developments on Sierpinski Patch Antenna........................................................ 31 
3.2 Design of the Stacked Sierpinski Antenna........................................................................ 34

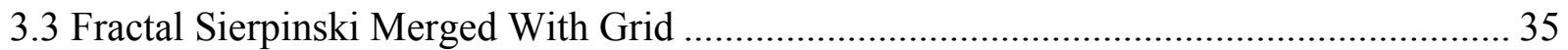

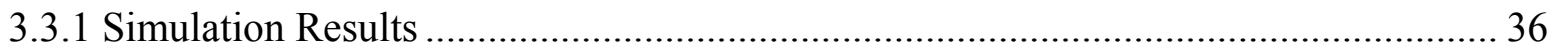

3.3.2 Comparison of Result .......................................................................................... 39

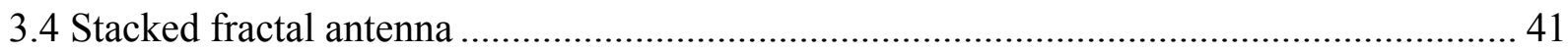

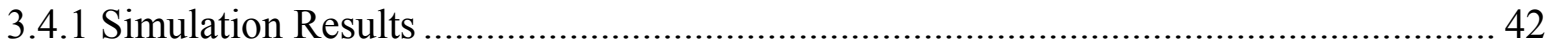

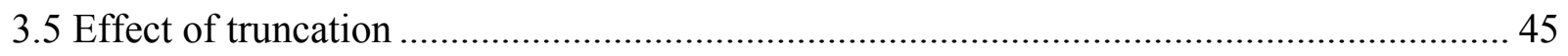

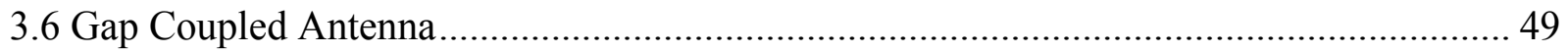

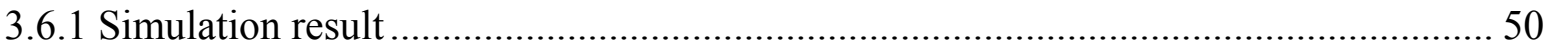

CHAPTER 4: CONCLUSION AND FUTURE RESEARCH …………………........................ 55

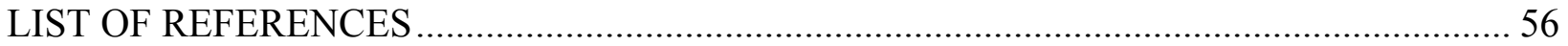




\section{LIST OF FIGURES}

Figure 1: Structures of classical fractals a) Cantor set, b) Koch curve, c) Sierpinski gasket, d)

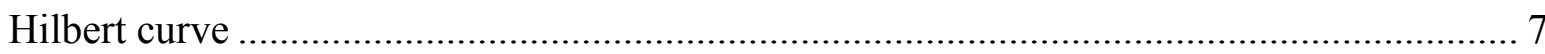

Figure2: Block diagram of the Iterated Function System (IFS) …………………………........ 8

Figure 3: Sierpinski obtained with three iterations............................................................... 9

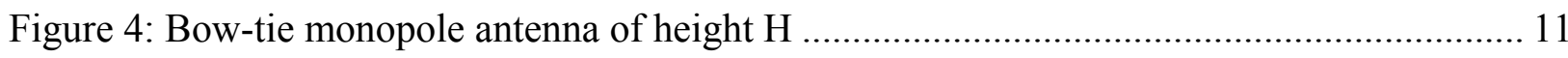

Figure 5: Characteristics of the Bowtie antenna with a height of 44mm. (a) return loss, (b), (c), and (d): Radiation pattern and current distributions at resonances $1.1 \mathrm{GHz}, 4.2 \mathrm{GHz}$, and

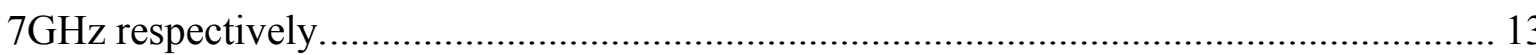

Figure 6: Characteristics of the bowtie antenna with a height of $22 \mathrm{~mm}$. (a) return loss, (b), and (c): Radiation pattern and current distributions at resonances $2.3 \mathrm{GHz}$, and $8.4 \mathrm{GHz}$ respectively. 15

Figure 7: Characteristics of the bowtie antenna with a height of $11 \mathrm{~mm}$. (a) return loss, (b) radiation pattern at $4.6 \mathrm{GHz}$ 16

Figure 8: Characteristics of Sierpinski monopole antenna with height 44mm. (a) return loss, (b), (c), (d), and (e): Radiation pattern at $1 \mathrm{GHz}, 3.6 \mathrm{GHz}, 7.7 \mathrm{GHz}$, and $13.2 \mathrm{GHz}$ respectively

Figure 9: Top view (a) and side view (b) of the Sierpinski patch antenna................................... 21

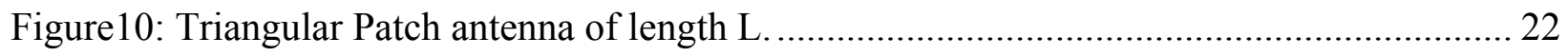


Figure 11: Characteristics of triangular patch antenna with length 44mm.(a) return loss, (b), (c), and (d) radiation pattern at $4.6 \mathrm{GHz}, 5.4 \mathrm{GHz}$ and $7.6 \mathrm{GHz}$ respectively. 24

Figure 12: Characteristics of triangular patch antenna with height $22 \mathrm{~mm}$. (a) return loss, (b), and (c) : Radiation pattern at $6.2 \mathrm{GHz}$, and $9.8 \mathrm{GHz}$ 26

Figure 13: Characteristics of triangular patch antenna with height $11 \mathrm{~mm}$. (a) return loss, (b) radiation pattern and current distribution at $10.2 \mathrm{GHz}$. 27

Figure14: Characteristics of Sierpinski fractal antenna. (a) Return loss, (b) radiation pattern at $10.3 \mathrm{GHz}$ 29

Figure 15: Current distributions at (a) $3.4 \mathrm{GHz} \&$ (b) $6.1 \mathrm{GHz}$. 30

Figure 16: Sierpinski patch antenna with grid (a) grid, (b) original fractal patch, (c) crosssectional view of the structure. 31

Figure 17: Return loss of Sierpinski patch antenna with grid.................................................... 32

Figure 18: Geometry and S11 characteristics of the modified patch antenna [23] ...................... 33

Figure 19: Geometry and S11 characteristics of modified Sierpinski grid antenna [18].............. 34

Figure 20: (a) Sierpinski fractal, (b) grid structure, and (c) side view of the overlapped fractal antenna 36

Figure 21: Characteristics of the merged Sierpinski fractal antenna. (a) Return loss, Radiation patterns and current distributions at (b) $5.2 \mathrm{GHz}$, (c) $6.2 \mathrm{GHz}$ and (d) $8.7 \mathrm{GHz}$. 38

Figure 22: Characteristics of the Sierpinski fractal antenna with grid. (a) Return loss (dotted line: Sierpinski with grid, solid line: merged Structure), radiation pattern and current distribution at (b) $5.2 \mathrm{GHz}$, (c) $6.6 \mathrm{GHz}$ and (d) $9 \mathrm{GHz}$ 41

Figure23: Stacked Fractal (a) top view, and (b) side view 42 
Figure 24: Characteristics of the Stacked Sierpinski fractal antenna. (a) Return loss, Radiation patterns and current distributions at (b) $4.5 \mathrm{GHz}$, (c) $5.1 \mathrm{GHz}$, (d) 6.2 and (e) $7.4 \mathrm{GHz}$.... 45

Figure 25:Layout of truncated stacked Sierpinski antenna (a) top view, and (b) side view......... 46

Figure 26 Characteristics of truncated Sierpinski fractal antenna. (a) Return loss, Radiation patterns and current distributions at (b) $4.9 \mathrm{GHz}$, (c) $5.6 \mathrm{GHz}$, (d) $6.5 \mathrm{GHz}$ and (e) $8.6 \mathrm{GHz}$

Figure 27: Layout of the gap coupled antenna.(a) top view, (b) side view. 50

Figure 28: Characteristics of the gap coupled stacked Sierpinski fractal antenna. (a) Return loss, Radiation patterns and current distributions at (b) $4.9 \mathrm{GHz}$, (c) $6 \mathrm{GHz}$, (d) $6.6 \mathrm{GHz}$, (e) 7.2 GHz, (f) $8 \mathrm{GHz}$, and (g) $8.7 \mathrm{GHz}$. 54 


\section{LIST OF TABLES}

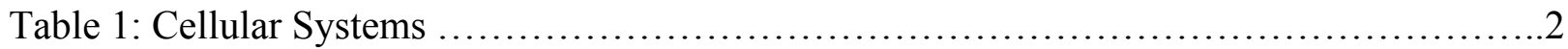




\section{CHAPTER ONE: INTRODUCTION}

\section{$\underline{1.1 \text { Overview of Wireless Systems }}$}

Wireless communications is a rapidly growing segment of the communications industry, with the potential to provide high-speed high-quality information exchange between portable devices located anywhere in the world. Potential applications enabled by this technology include cellular phones, multimedia Internet-enabled cell phones, smart homes and appliances, automated highway systems, video teleconferencing and distance learning, and autonomous sensor networks, to name just a few. Wireless communications network have become more pervasive than anyone could have imagined when the cellular concept was first developed in the 1960s and 1970s. Major mobile or cellular network are mentioned below.

The first generation American cellular system was the Advanced Mobile Phone System (AMPS). The AMPS was released in 1983 using the $800 \mathrm{MHz}$ to $900 \mathrm{MHz}$ frequency band and the $30 \mathrm{KHz}$ bandwidth for each channel. It uses analog technology with frequency division multiple access (FDMA) and carries voice only. Although AMPS has been a commercial success, it lacks security and capacity. The AMPS was later enhanced to NAMPS (Narrowband AMPS) and D-AMPS (Digital AMPS).

GSM (Global System for Mobile Communication) is a digital TDMA system that utilizes eight time slots of $25 \mathrm{KHz}$ each. GSM is popular in Europe and Asia. GSM network in United States operates at $850 \mathrm{MHz}$ (Cell band) and $1900 \mathrm{MHz}$ band (PCS band) whereas in other countries operates at $900 \mathrm{MHz}$, and $1900 \mathrm{MHz}$ (DCS band) band. The 2.5G GSM is GRPS 
(General Package Radio Service), which is then going to evolve to EDGE (Enhanced data rate for GSM Evolution), a 3G technology.

CDMA (Code Division Multiple Access) is the fastest growing wireless service and most popular in United States. It is also known as IS-95.CDMA and offers greater capacity than its competitors. It operates on $800 \mathrm{MHz}$ and $1900 \mathrm{MHz}$ band WCDMA (also called Universal Mobile Telecommunication System) is $3 \mathrm{G}$ System and will be operating on $2000 \mathrm{MHz}$ band. CDMA 2000 1x, CDMA20001xEV are higher generation of CDMA. Major second generation systems deployed are described in Table 1.1.

Table 1: Cellular systems

\begin{tabular}{|l|l|l|l|}
\hline & Purpose & \multicolumn{2}{l|}{ Frequency } \\
\hline GSM & $\begin{array}{l}\text { Digital cellular phone system; } \\
\text { mainly used worldwide }\end{array}$ & U.S & $\begin{array}{l}850 \mathrm{MHz}(\mathrm{Cell} \text { band), } \\
1900 \mathrm{MHz} \text { (PCS) }\end{array}$ \\
\cline { 3 - 4 } & & ROW & $\begin{array}{l}900 \mathrm{MHz}, \\
1800 \mathrm{MHz}(\mathrm{DCS})\end{array}$ \\
\hline CDMA (IS-95) & $\begin{array}{l}\text { Second generation CDMA based } \\
\text { network. }\end{array}$ & $\begin{array}{l}850 \mathrm{MHz} \text { Cell band), } \\
1900 \mathrm{MHz} \text { (PCS) }\end{array}$ \\
\hline WLAN & Wireless internet access & $2.4 \mathrm{GHz}, 5.2 \mathrm{GHz}$ \\
\hline
\end{tabular}

The types of antenna that are commercially used for these different wireless systems are determined by the cost, size and the performance.

\section{$\underline{1.2 \text { Antennas for Wireless Systems }}$}

There has been increasing need for innovative antennas on both mobile terminal and base stations in wireless communication including the increasingly popular wireless local area 
network (WLAN) system in the $2.4 \mathrm{GHz}$ (IEEE $802.11 \mathrm{~b} ; 2400-2484 \mathrm{MHz}$ ) and $5.2 \mathrm{GHz}$ (IEEE 802.11a; 5150-5350 MHz) bands.

Commonly used antennas in wireless devices are either external or internal. External antennas can be fixed or retractable. The main disadvantage is their fragileness, susceptibility to damage and possible human absorption of radiation. The most commonly used external antennas are the whip, monopole and helix. The conventional monopole usually being a quarter wavelength long, is wound in the form of helix or Stacked loop to occupy less space. Internal antennas, on the other hand, are fabricated directly on the circuit board of the wireless systems. The ground plane of PCB forms the ground plane of antenna and hence prevents backward radiation.

Constrains on wireless antenna design include requirement for multi-band resonance. Dual band, tri band, quad band, and dual mode cellular phone is an example of a multiple frequency wireless devices. Dual band operation involves transmitting and receiving in both $850 \mathrm{MHz}$ and $1900 \mathrm{MHz}$ frequencies in GSM mode where as dual mode involves operating in both CDMA and GSM modes. Traditional antennas are unable to meet these requirements and hence alternative approaches are needed. Recent antenna developments have applied fractal mathematics to antenna design, resulting in new fractal antennas with multi-band behavior.

\section{$\underline{1.3 \text { Fractal Overview }}$}

A fractal is a rough or fragmented geometric shape that can be subdivided in parts, each of which is (at least approximately) a reduced-size copy of the whole. Fractals are generally selfsimilar and independent of scale. There are many mathematical structures that are fractals; e.g. Sierpinski's gasket, Cantor's comb, von Koch's snowflake, the Mandelbrot set, the Lorenz 
attractor, etc [1]. Fractals also describe many real-world objects, such as clouds, mountains, turbulence, and coastlines that do not correspond to simple geometric shapes. The terms fractal and fractal dimension are due to Mandelbrot, who is the person most often associated with the mathematics of fractals [Mandelbrot, 1983]. Mandelbrot included a definition of fractal dimension (of a geometric object) when he first talked about the concept of fractal in 1977 [Lauwerier, 1991].

Fractals have found applications in geography, biology, and engineering $[1,2]$. Fractals in engineering have been used in the design of antennas, frequency selective surfaces, and the image processing $[2,3]$. Jaggard et al have investigated the fractal frequency selective surfaces and their effects on scattering $[3,4,5]$. Romeu et al. have studied the frequency behavior of fractal shaped antennas [6-12]. Werner et al. have investigated the radiation property of fractal arrays [13-15].

Regarding antennas, fractal antenna theory is a relatively new area. However, Fractal antennas and its superset fractal electrodynamics [Werner and Mittra, 1999] is a hotbed of research activity. The geometry of the fractal antenna provides an attractive multi-band solution. One should expect a self-similar antenna (which contains many copies of itself at several scales) to operate in a similar way at several wavelengths. That is, the antenna should maintain similar radiation characteristics through several corresponding bands.

Several fractal antennas geometries have been introduced and analyzed. One such example is Sierpinski type of fractal geometry, which has been used to design both monopole and patch type antennas $[9-12,16]$. Several structures are derived from the original Sierpinski fractal structure and analyzed in order to get better multi-band behavior [17-19]. The monopole type Sierpinski antenna exhibits well-defined multi-band characteristics however; the Sierpinski 
patch antenna does not perform as well as the monopole. Several modifications have been reported to improve the performance of the antenna [20-23].

\subsection{Research focus and thesis overview}

The focus of this thesis is to study monopole and patch type Sierpinski antennas and includes modifications to improve the behavior of conformal patch design. Chapter 2 discusses both these antennas and summaries the recent developments in the area. Chapter 3 discusses the modification included in the classic Sierpinski fractal patch antenna for improved performance and Chapter 4 gives a summary and conclusions. 


\section{CHAPTER TWO: FRACTAL ANTENNAS}

\section{$\underline{2.1 \text { Classical Fractals }}$}

Fractals have a long history in mathematics. The Koch curve, Sierpinski gasket, and Peano curve are three of the more famous fractals and they were proposed during early $20^{\text {th }}$ century [1]. These fractal objects have similar scale lengths with infinite levels of details. Figure 1 displays examples of several classical fractals.

The Cantor set is the set of multiple intervals with alternating gaps and it is important in set theory and in dynamical systems. The Koch curve was constructed as a study of limits, with the ideal curve containing infinite number of sharp corners and therefore nowhere differentiable. The Hilbert curve is another meandering curve and its use includes image processing such as dithering to control shading and the Sierpinski gasket results from the continual removal of an inverted triangle within a larger triangle.

(a) 


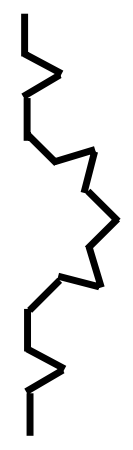

(b)

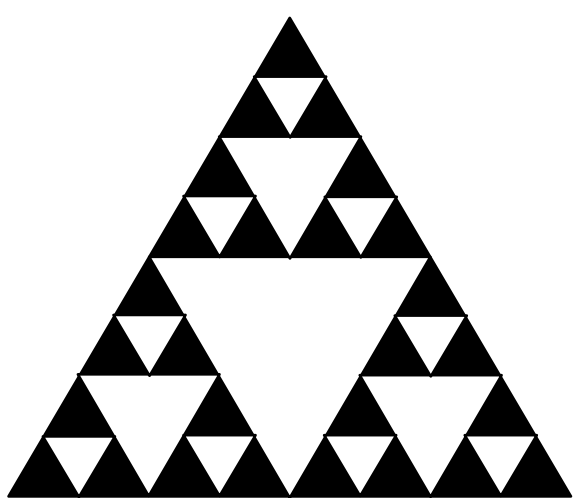

(c)

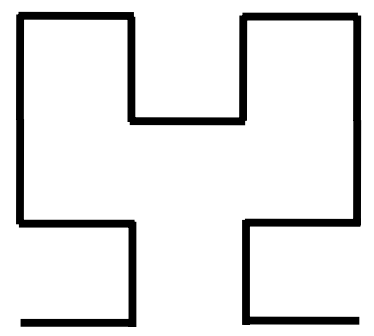

(d)

Figure 1: Structures of classical fractals a) Cantor set, b) Koch curve, c) Sierpinski gasket, d) Hilbert curve

\subsection{Iterated Function System}

Fractals have self-similar objects at multiple scale lengths organized in a deterministic or stochastic way. The creation of fractals is based on set theory and can be produced by simple feedback process. The fractal generation procedure begins with a generator shape, which is input into a mapping function, and its output becomes the input for the next iteration and this is called the Iterated Function System (IFS). Figure 2 gives the general idea of the iterated function system. 
The feedback loop mapping function can be of either the linear or the nonlinear type. Linear or affine transformation consists of scaling and translation and preserves the general overall shape. On the contrary, nonlinear transformation distorts any linear structure and results in a different shape.

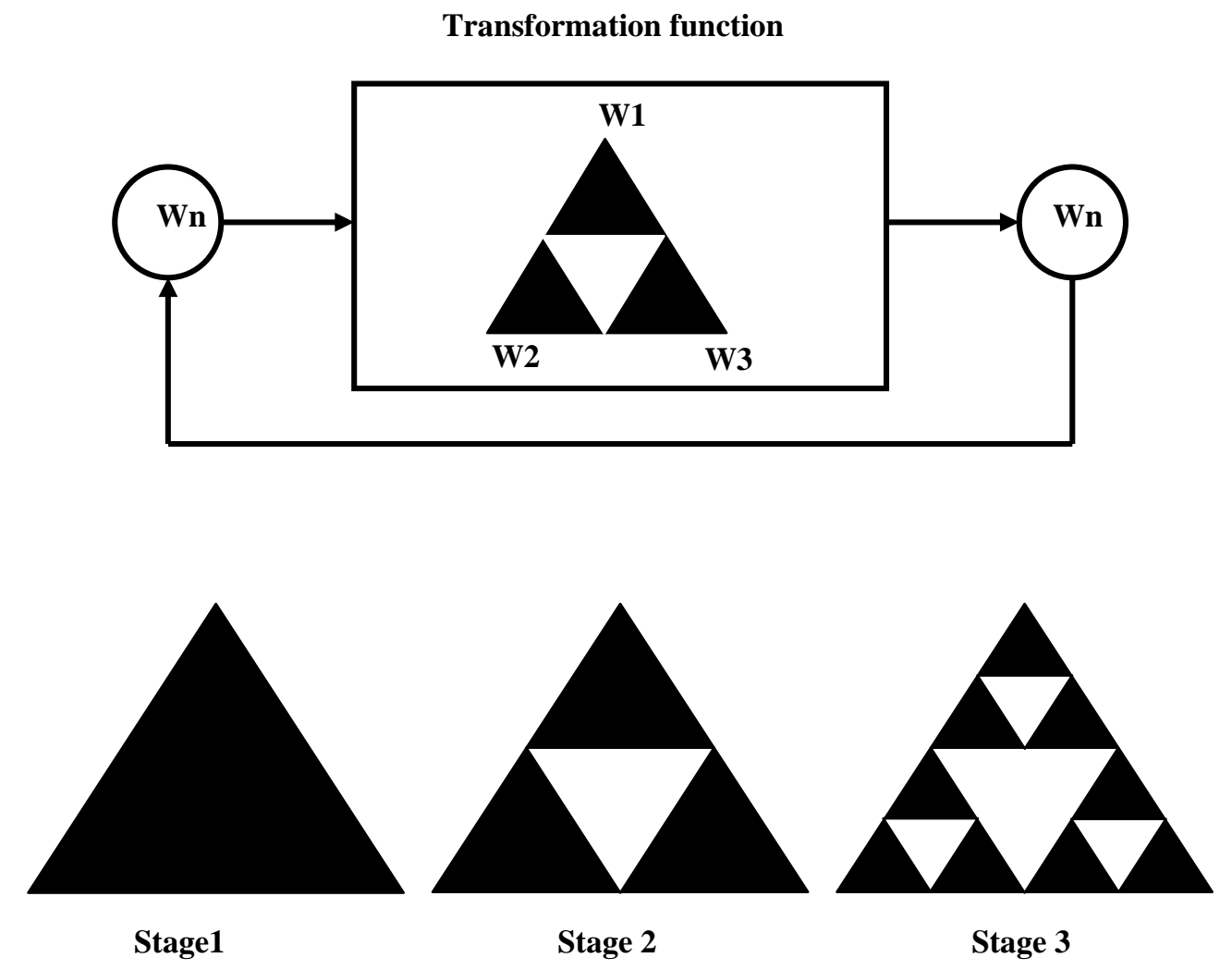

Figure2: Block diagram of the Iterated Function System (IFS)

\section{$\underline{2.3 \text { Sierpinski Fractal Antenna }}$}

Recent efforts by several researchers around the world to combine fractal geometry with electromagnetic theory have led to a plethora of new and innovative antenna designs. Fractals have no characteristic size, and are generally composed of many copies of themselves at different scales. These unique properties of fractals have been exploited in order to develop a 
new class of antenna element designs that are multi-band. The Sierpinski fractal antenna is the most explored antenna among all the fractal structures.

In this Chapter, monopole and patch configurations of Sierpinski fractal antennas are presented. These were simulated using IE3D and analyzed in order to obtain a better understanding of the antenna's fractal behavior. The results obtained in each case are compared with those published in the literature.

The Sierpinski gasket is named after the Polish mathematician Sierpinski who described some of the main properties of the fractal shape in 1916. The original gasket is constructed by subtracting a central inverted triangle from a main triangle shape. After the subtraction, three equal triangles remain on the structure, each one being half of the size of the original one. One can iterate the same subtraction procedure on the remaining triangles and if the iteration is carried out an infinite number of times, the ideal fractal Sierpinski gasket is obtained [1,9].

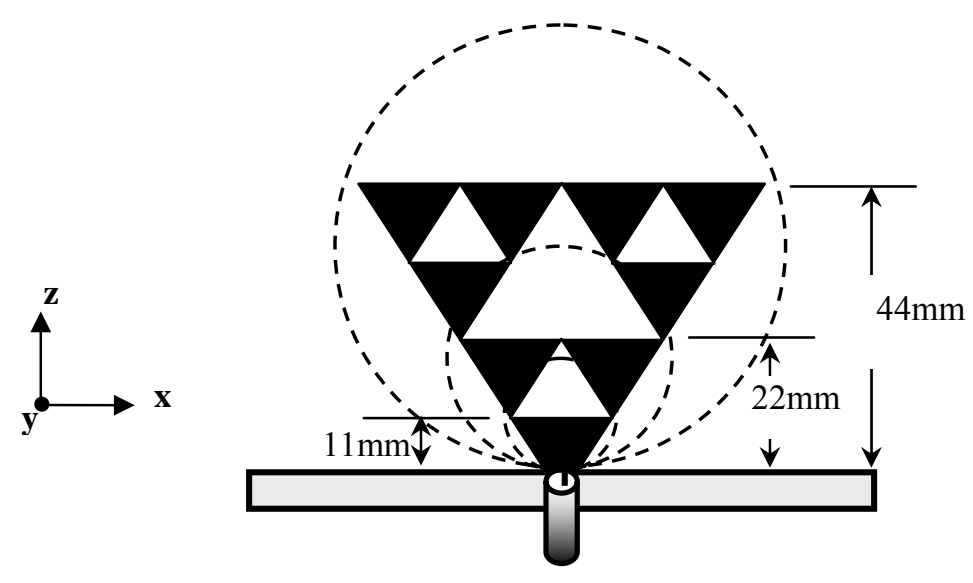

Figure 3: Sierpinski obtained with three iterations. 


\subsection{Sierpinski Fractal As Monopole}

A third order Sierpinski gasket constructed through three iterations with a scale factor of 2 is shown in Figure 3. The height of the Sierpinski fractal is chosen as $44 \mathrm{~mm}$ based on fractal used on the literature [9, 23]. Three scaled versions of the Sierpinski gasket, Sierpinski subgaskets, fed simultaneously at the apex are found on the antenna and are shown inside the circles in the Figure 3. The first Sierpinski sub-gasket is third order Sierpinski of height $11 \mathrm{~mm}$, the second sub-gasket is second order Sierpinski of height $22 \mathrm{~mm}$ and the third is first order Sierpinski of height $44 \mathrm{~mm}$. If one neglects the contribution of the center holes to the antenna performance and admits that the current flowing from the feeder should concentrate over a region that is comparable in size to the wavelength, a behavior similar to three scaled bow-tie monopole antennas with heights equal to those of sub-gaskets could be expected. i.e., the size of the three bow-tie antennas would be 11, 22 and 44mm [9, 23]. Based on this assumption, we can say that at higher frequencies, the current should concentrate on smallest sub-gasket because it is equivalent to the bowtie monopole of height $11 \mathrm{~mm}$ which would resonate at higher frequency compared to bowtie monopole of $22 \mathrm{~mm}$ and $44 \mathrm{~mm}$. Below, we study the similarities in the behavior of the bowtie monopole antenna and the Sierpinski monopole antennas.

Bowtie monopoles corresponding to each sub-gasket were simulated and their results are compared with the third order Sierpinski fractal antenna as published in [9]. Figure 4 shows a bow-tie monopole antenna of height $\mathrm{H}$, where $\mathrm{H}=11 \mathrm{~mm}, 22 \mathrm{~mm}$, or $44 \mathrm{~mm}$, corresponding to each sub-gasket. 


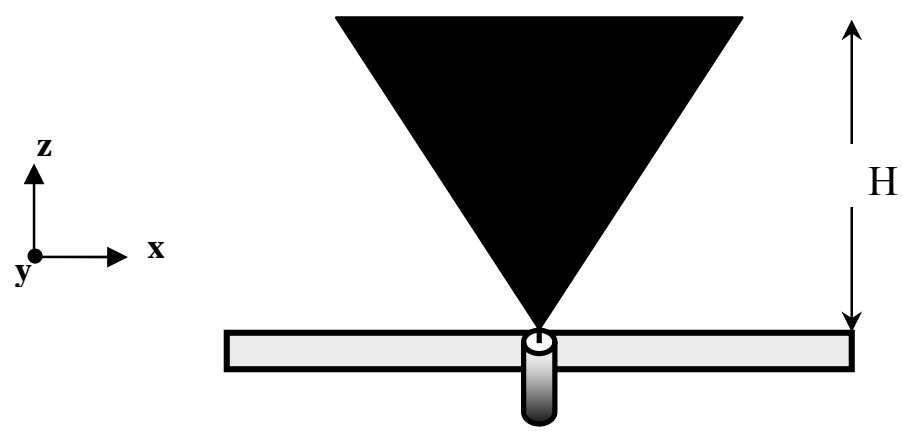

Figure 4: Bow-tie monopole antenna of height $\mathrm{H}$.

\subsubsection{Simulated Results for Bowtie antenna}

Figure 5 shows the results of the bowtie antenna with a height $44 \mathrm{~mm}$, which corresponds to the first sub-gasket. Five resonances at 1.1, 4.2, 7, 10.3 and $13 \mathrm{GHz}$ as shown in Figure 5a were obtained. The first resonance is due to the propagation of the first mode whereas the others are due to different higher mode propagation. The radiation pattern at $1.1 \mathrm{GHz}$ is perfectly omnidirectional as expected from a monopole structure, the pattern at $4.2 \mathrm{GHz}$ is a little distorted and at $7 \mathrm{GHz}$ has a notch in the main lobe. It is seen that higher modes start to propagate as the antenna; the side-lobes begin to increase. This is seen more clearly at resonances of 10.3 and $13 \mathrm{GHz}$. This is due to the fact that the wavelength of the resonating frequency is smaller than the effective length of the antenna. This effect is similar to that seen in monopole with length longer than quarter of the wavelength where we can see more side lobes as the length increases. We want the fractal antenna to operate in the first mode with good omni-directional radiation pattern; however the second resonance has a better match than the first resonance, so we can expect the second resonance to be dominant in the fractal. 


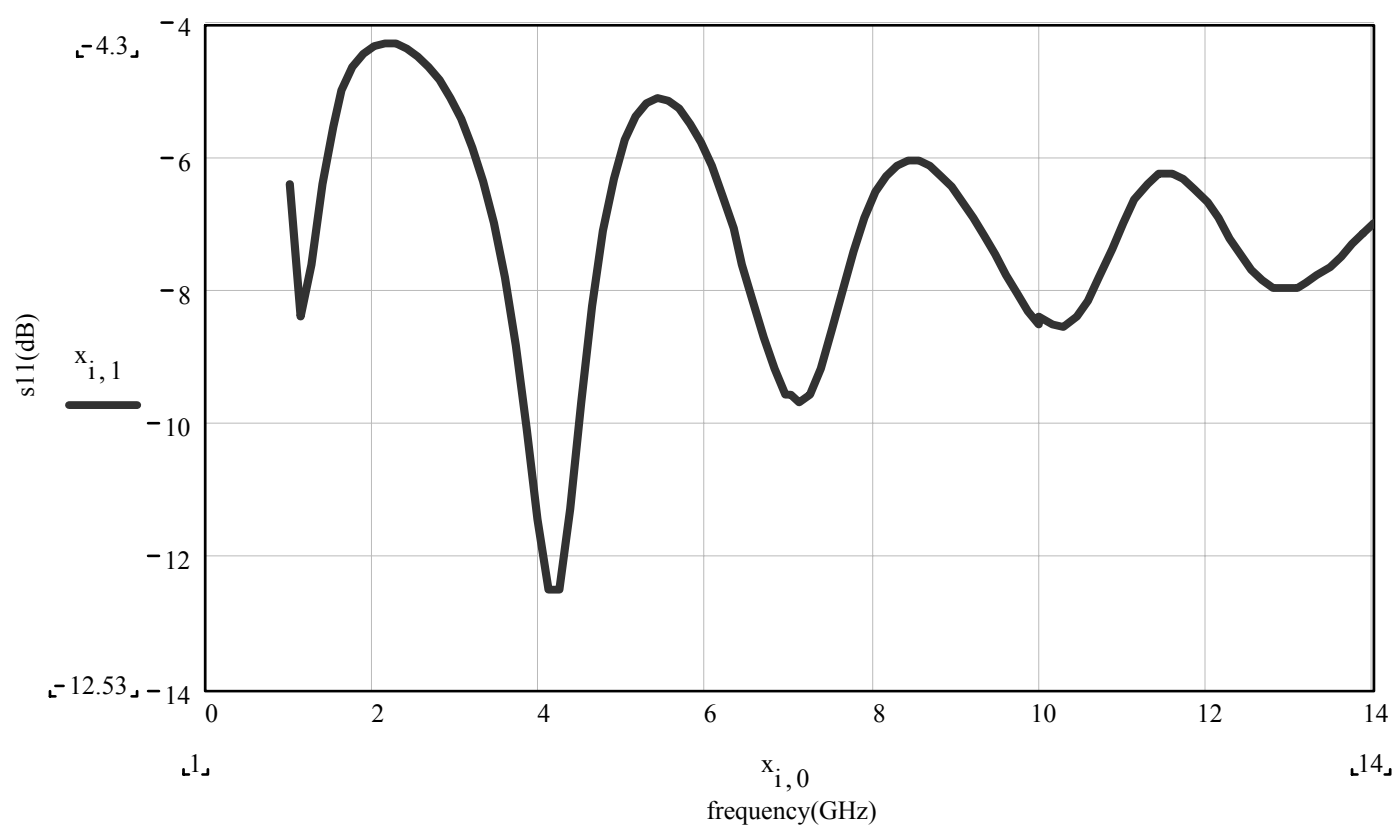

(a)
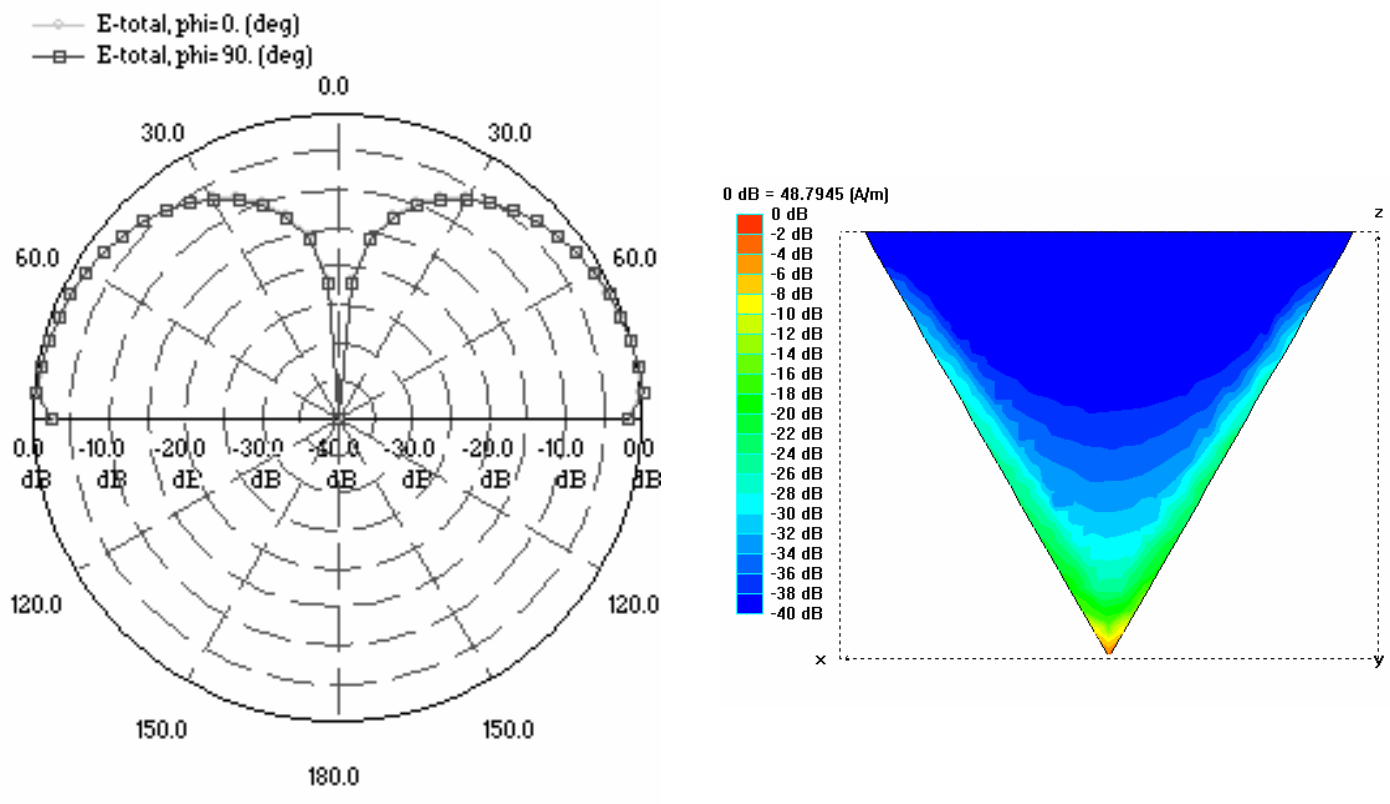

Polar Elevation Pattem

(b) 


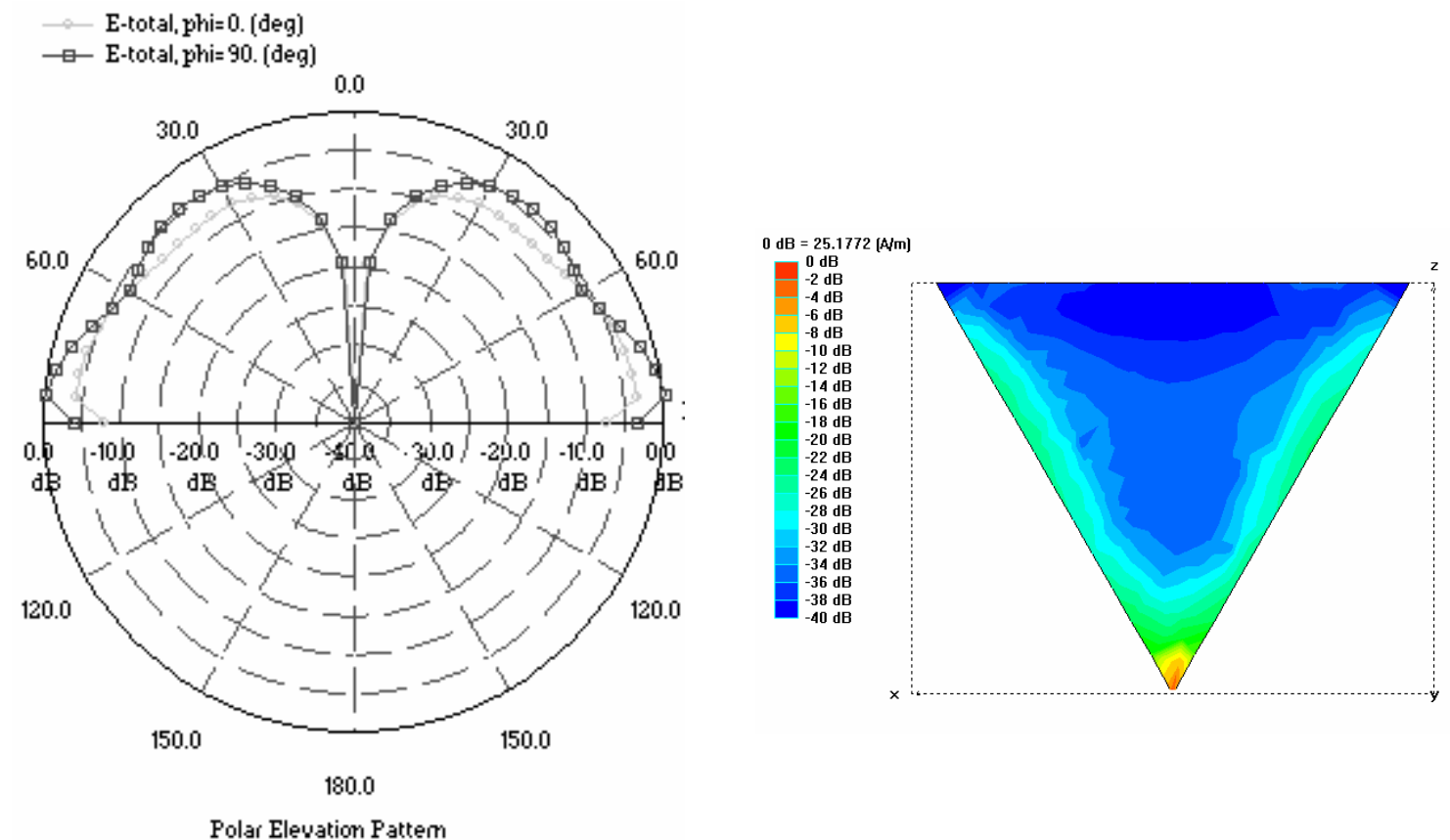

(c)

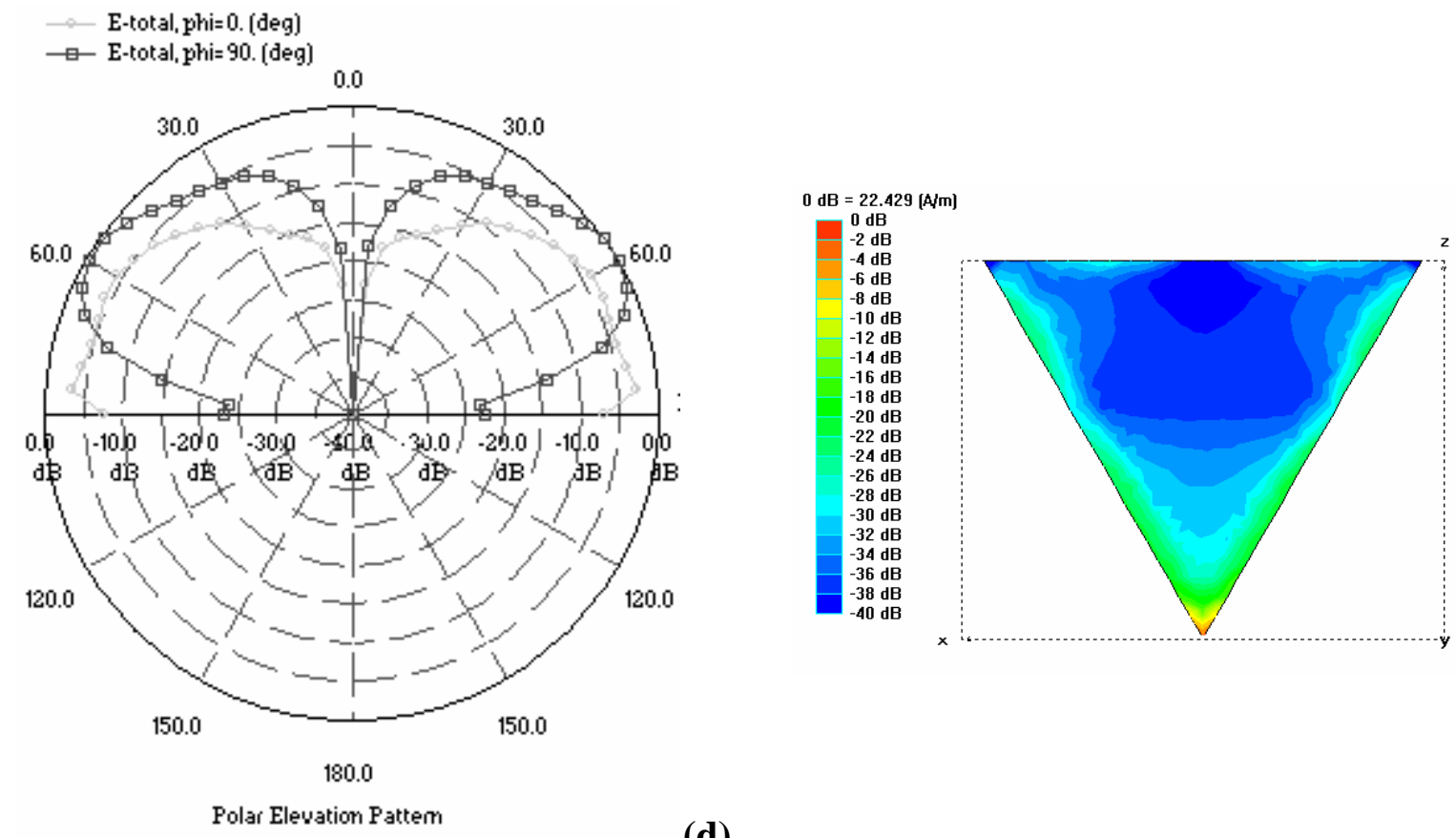

(d)

Figure 5: Characteristics of the Bowtie antenna with a height of 44mm. (a) return loss, (b), (c), and (d): Radiation pattern and current distributions at resonances $1.1 \mathrm{GHz}, 4.2 \mathrm{GHz}$, and $7 \mathrm{GHz}$ respectively. 
Figure 6 shows the simulated result of the bowtie antenna with a height of $22 \mathrm{~mm}$ in monopole arrangement. Two resonances are seen at 2.3 and $8.4 \mathrm{GHz}$. First resonant is due to the first mode propagation and the other is due to the second order mode. Just as was seen with the first sub gasket, the radiation pattern at $2.3 \mathrm{GHz}$ is flawlessly omni-directional whereas pattern at the $8.4 \mathrm{GHz}$ is little distorted. In terms of matching, it is seen that second resonance is matched better than the first one.

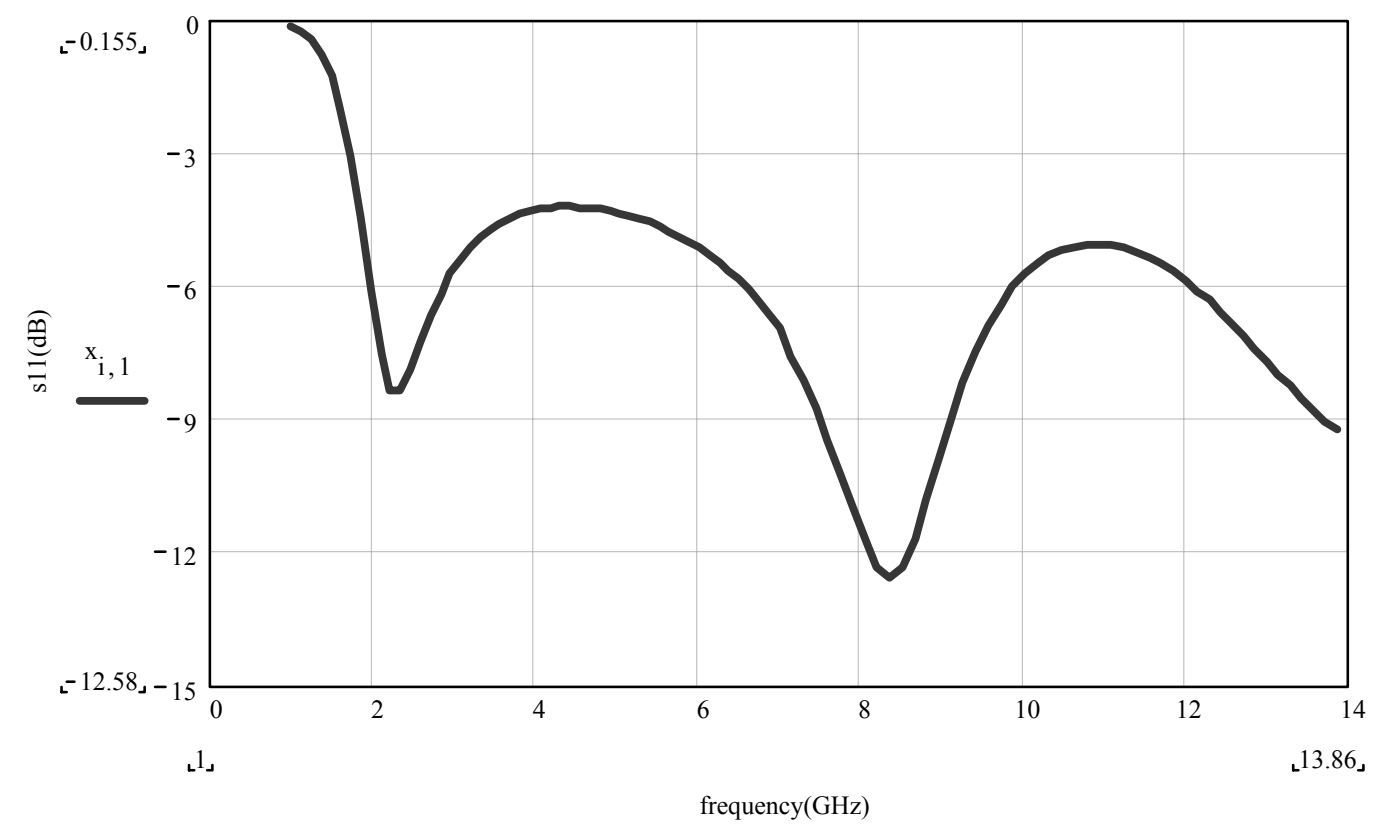

(a) 


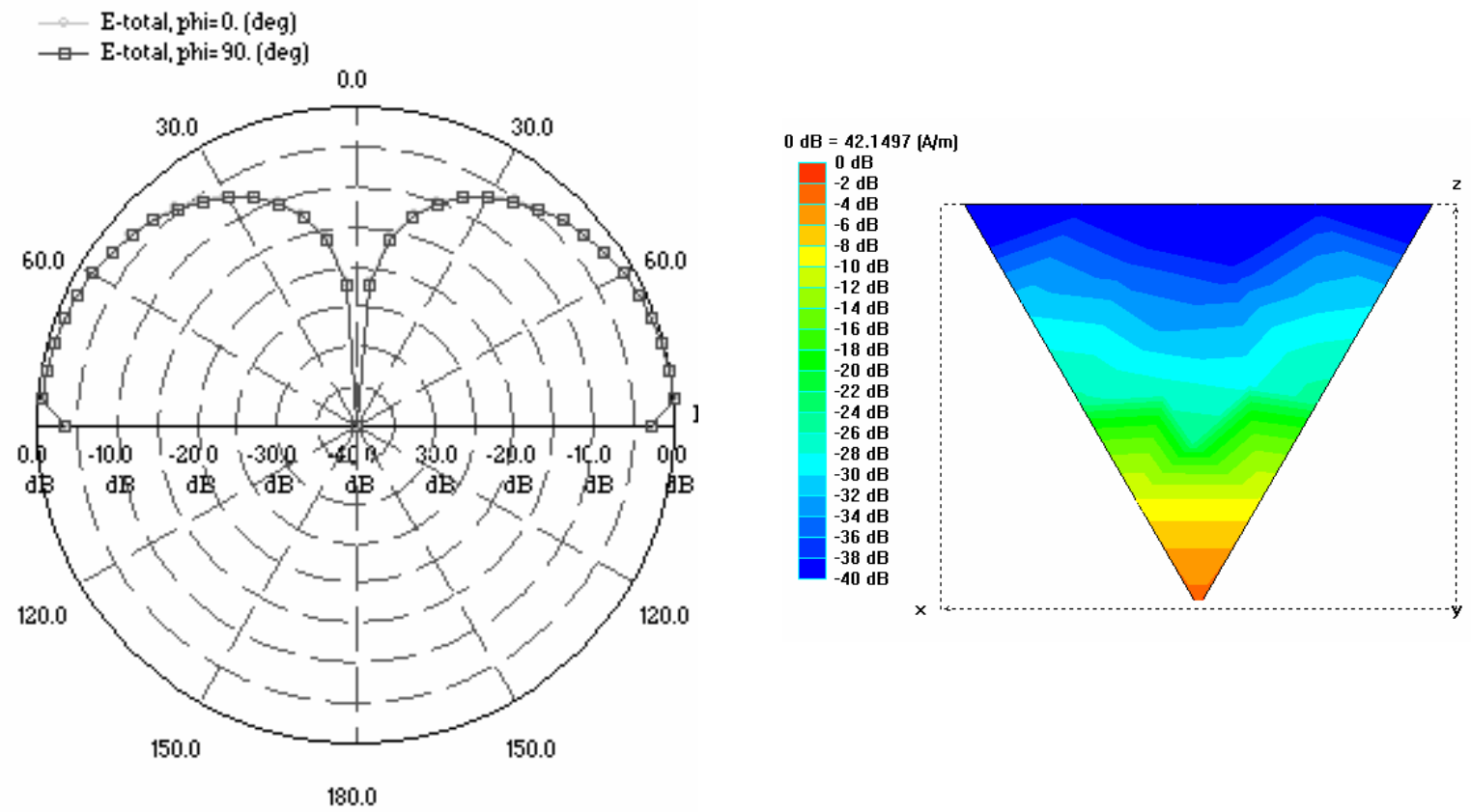

(b)
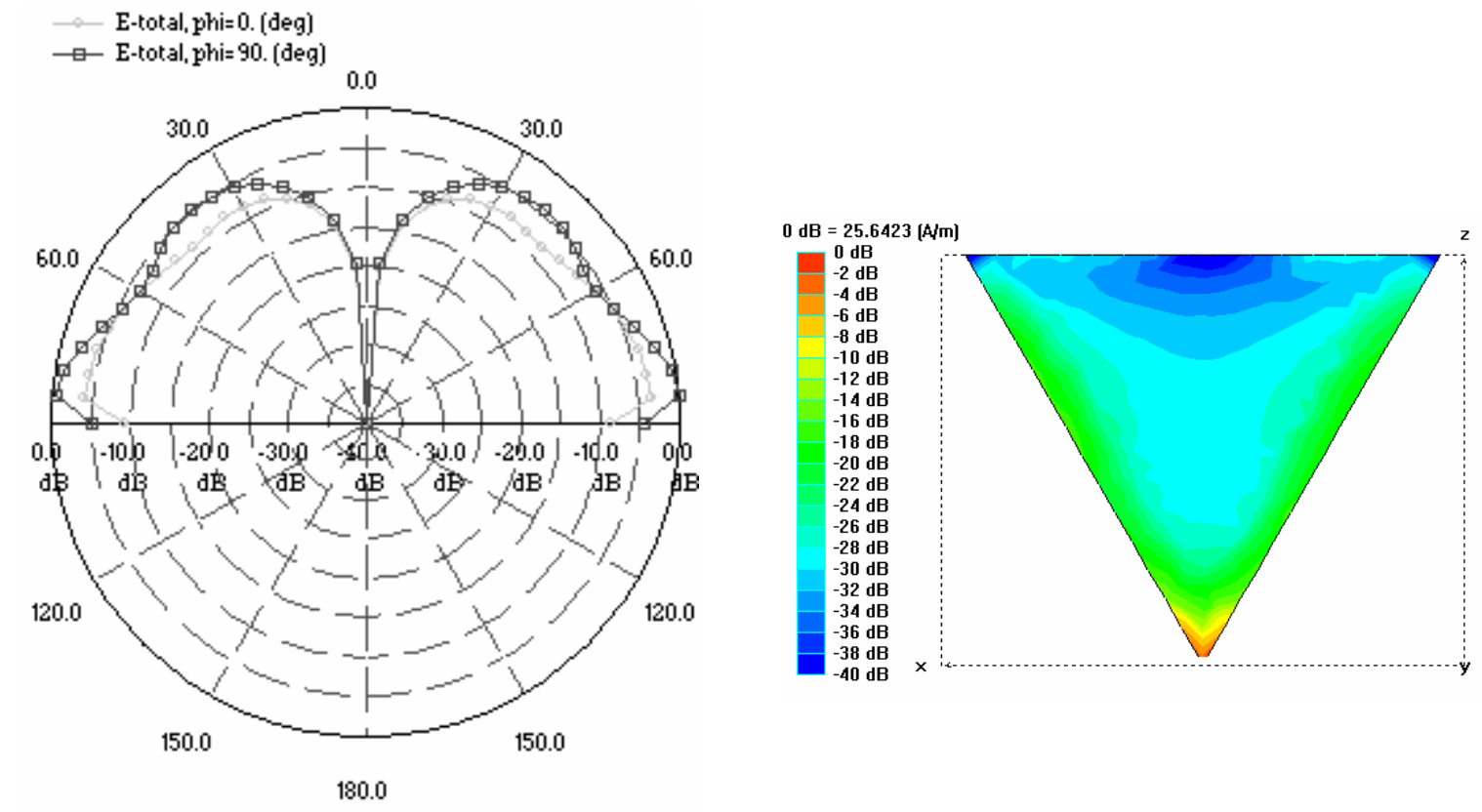

Polar Elevation Pattem

(c)

Figure 6: Characteristics of the bowtie antenna with a height of $22 \mathrm{~mm}$. (a) return loss, (b), and (c): Radiation pattern and current distributions at resonances $2.3 \mathrm{GHz}$, and $8.4 \mathrm{GHz}$ respectively. 
Figure 7 shows the simulated result of the bowtie antenna with a height of $11 \mathrm{~mm}$. One resonance is seen at $4.6 \mathrm{GHz}$. The radiation pattern is omni-directional.

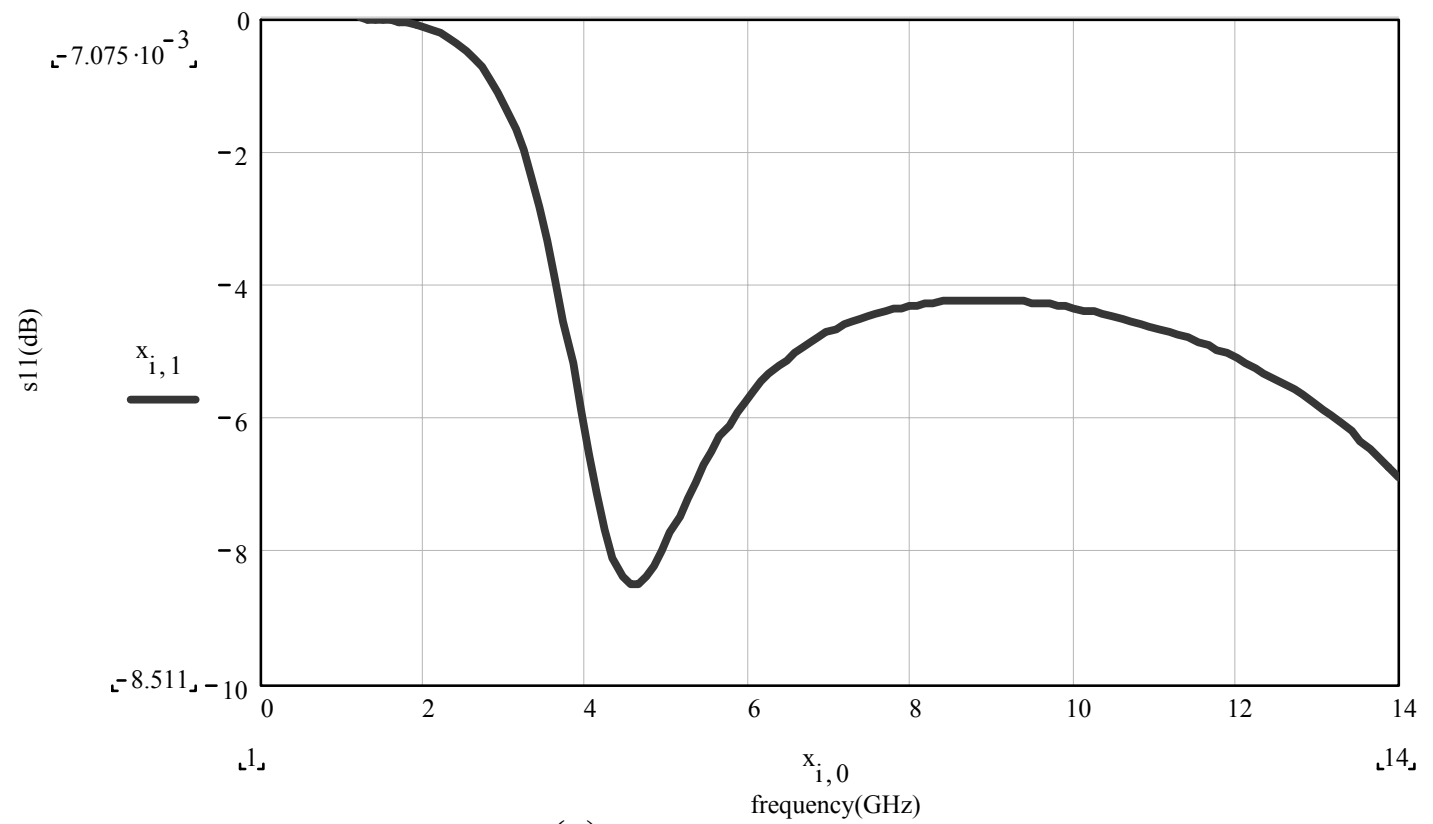

(a)
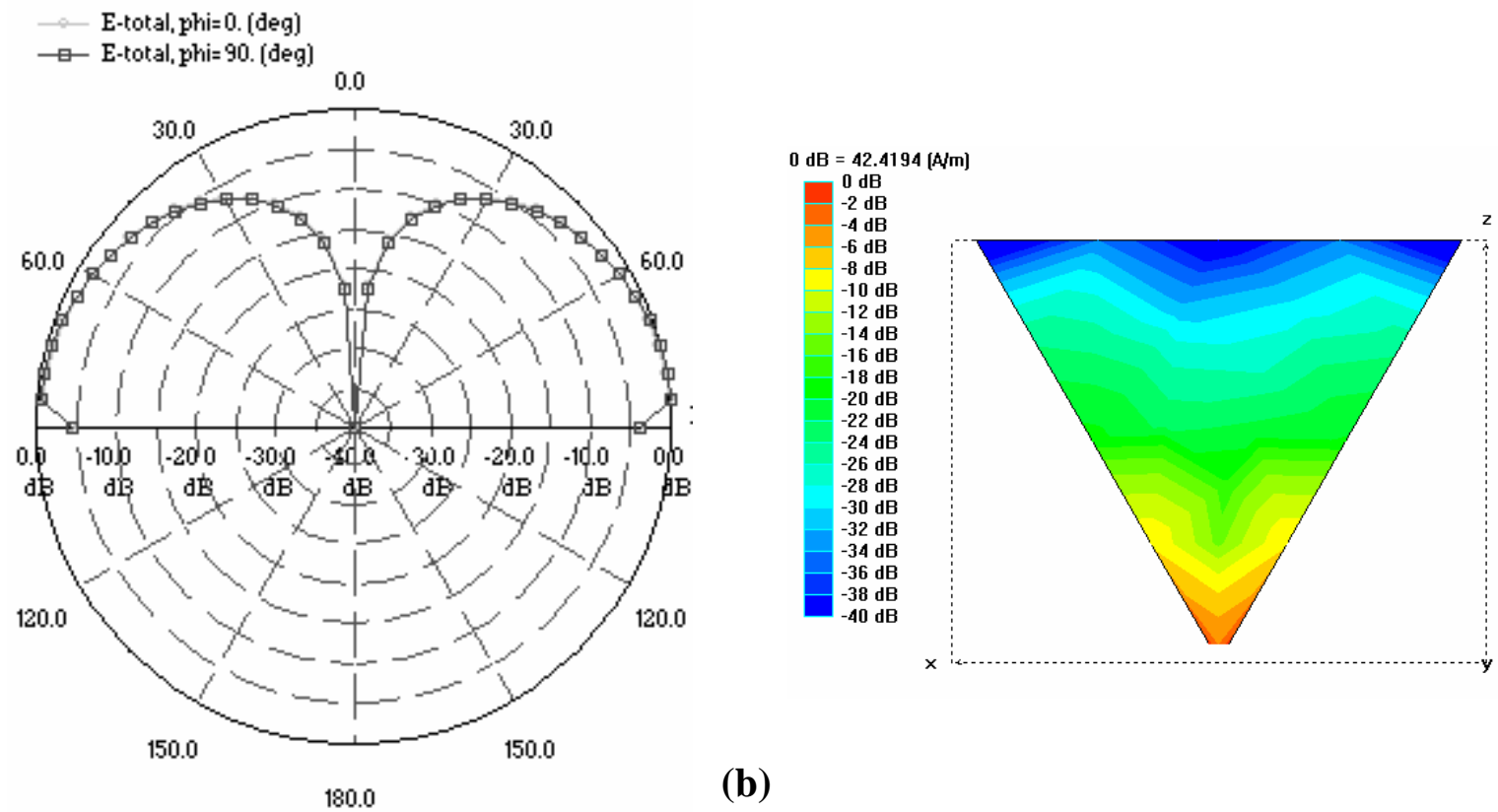

Figure 7: Characteristics of the bowtie antenna with a height of $11 \mathrm{~mm}$. (a) return loss, (b) radiation pattern at $4.6 \mathrm{GHz}$ 


\subsubsection{Simulated results for the Sierpinski monopole antenna}

The Sierpinski monopole antenna, as shown in Figure 3 was constructed in IE3D and simulated. It is shown that the antenna is matched approximately at frequencies

$$
\mathrm{f}_{\mathrm{n}} \approx \mathrm{c} / \mathrm{h} \delta^{\mathrm{n}}
$$

where $\mathrm{c}$ is the speed of light in vacuum, $\mathrm{h}$ is the height of the largest gasket, $\delta$ is the scale factor and $\mathrm{n}$ is a natural number [9]. The scale factor among the three sub-gaskets is $\delta=2$, therefore, one should look for similarities at frequencies also spaced by a factor of two. As expected, the Sierpinski monopole presents a log periodic behavior with three frequency bands spaced by a factor of 2 approximately. In the previous section, we saw that the antenna is better matched at the second resonance than the first one, so higher (2nd) mode resonance is expected in Sierpinski fractal antenna.

At higher resonances, 7.7 and $13.2 \mathrm{GHz}$, current should concentrate on the smallest subgasket of height $11 \mathrm{~mm}$, i.e. the triangle near the apex, because its length is comparable to the corresponding wavelengths. But we can see that current leaks to the upper triangles of bigger sub-gaskets, generating higher mode propagation as seen in Figure 8. Therefore, the radiation pattern is not perfectly omni-directional as expected. 


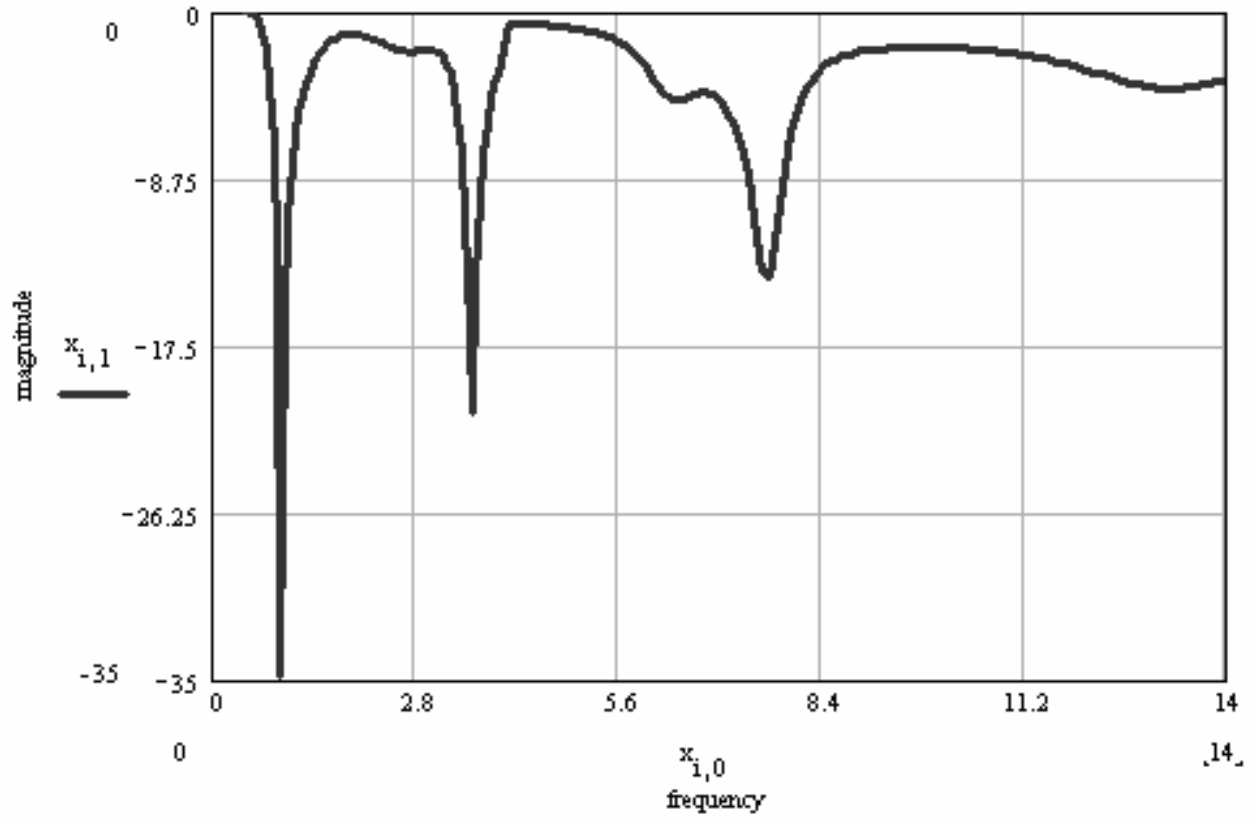

(a)
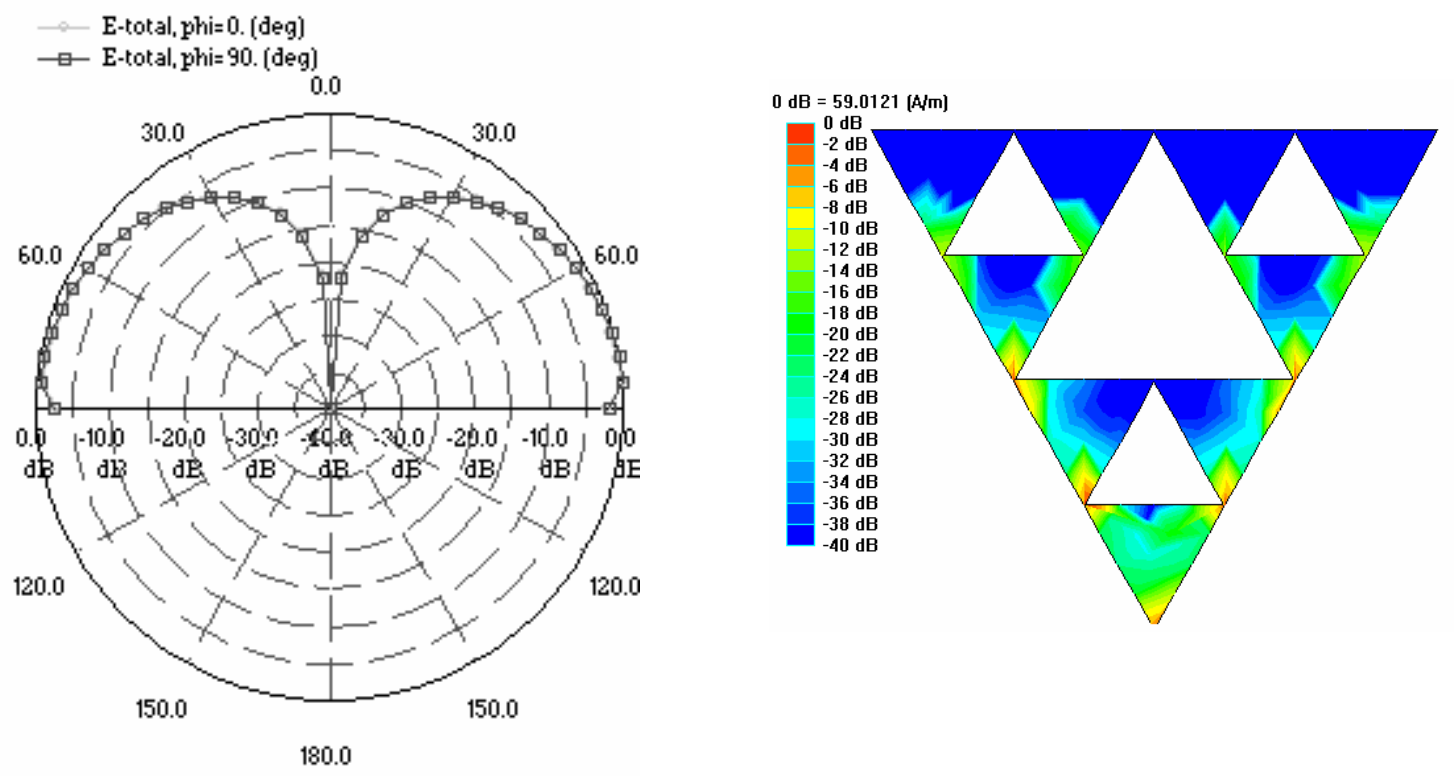

Polar Elevation Pattem

(b) 


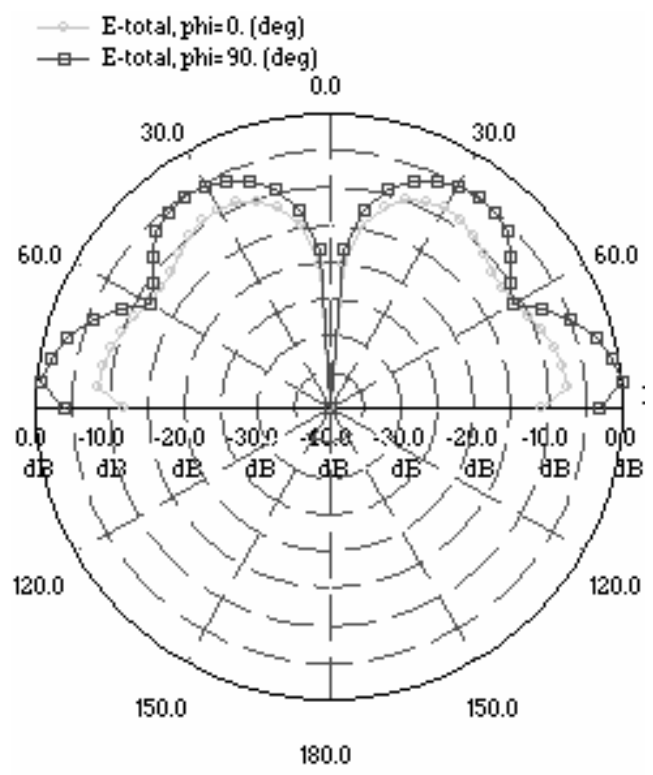

Polar Elevation Pattem

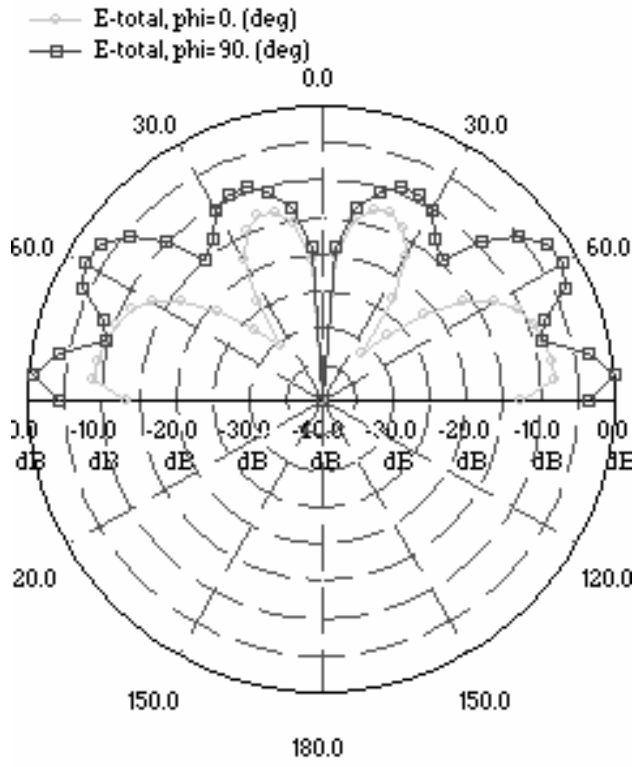

Polar Elevation Pattem

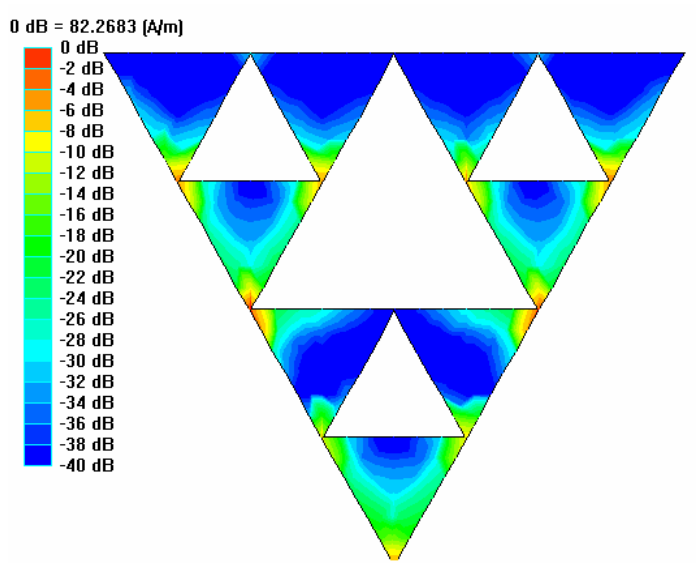

(c)

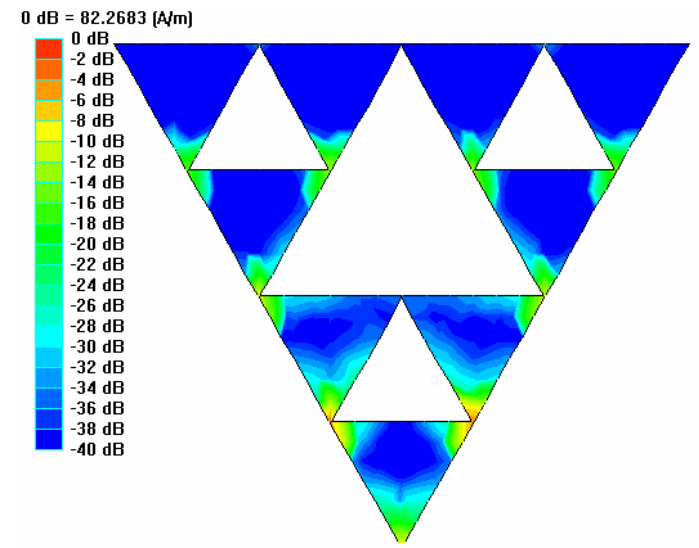

(d) 


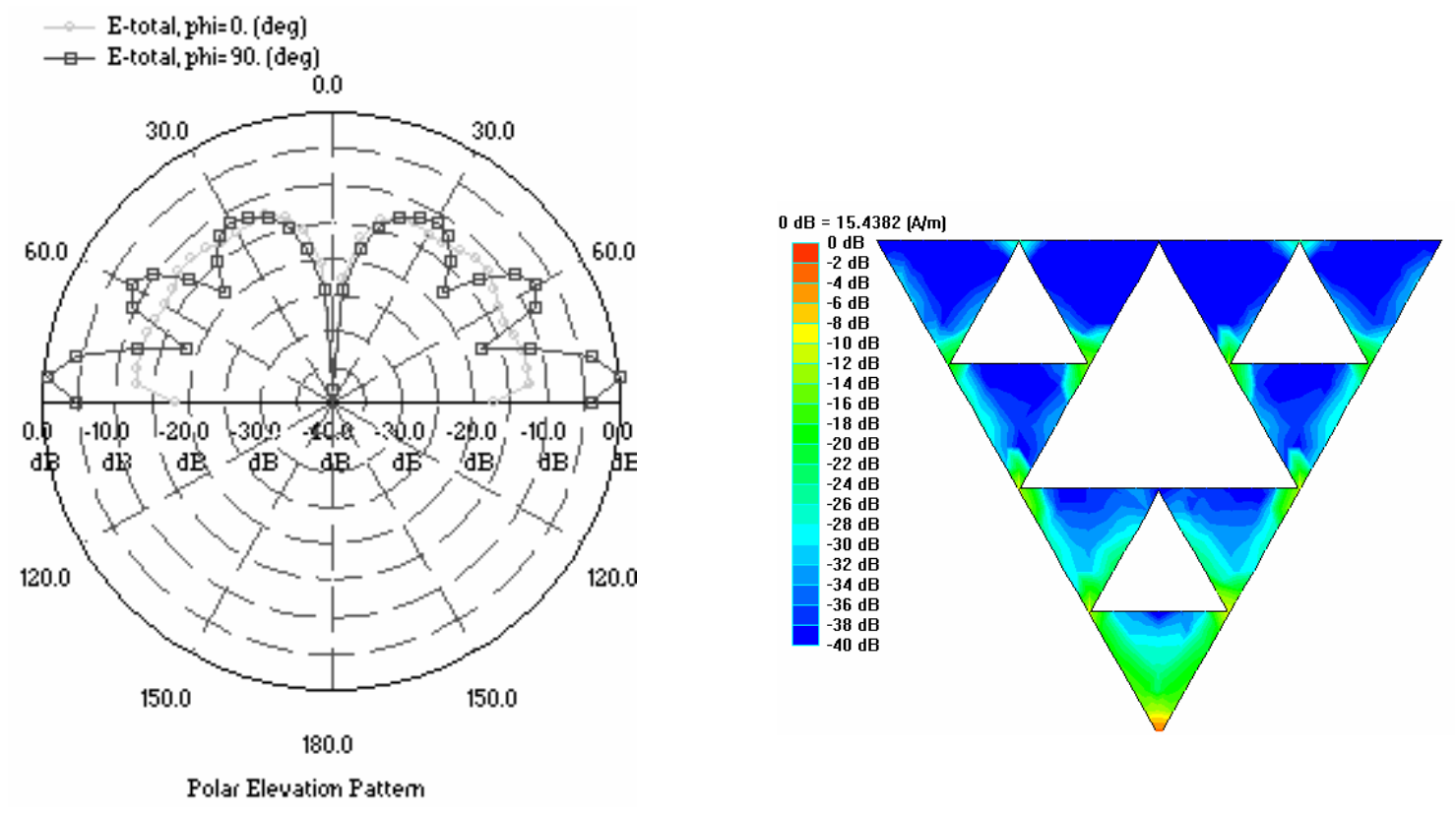

(e)

Figure 8: Characteristics of Sierpinski monopole antenna with height $44 \mathrm{~mm}$. (a) return loss, (b), (c), (d), and (e): Radiation pattern at $1 \mathrm{GHz}, 3.6 \mathrm{GHz}, 7.7 \mathrm{GHz}$, and $13.2 \mathrm{GHz}$ respectively.

The next step is to perform the same set of analysis done here for monopole Sierpinski antenna with the patch Sierpinski antenna and compare the results to the monopole Sierpinski antenna.

\section{$\underline{2.5 \text { Sierpinski Fractal Patch Antenna }}$}

The Sierpinski patch antenna is shown in Figure 9. The antenna has a $3 \mathrm{~mm}$ thick substrate with dielectric constant of 2.2. The height of the Sierpinski fractal is $44 \mathrm{~mm}$, same as in the previous section. The fractal antenna can be divided into three scaled version of Sierpinski fractal antenna, the Sierpinski sub-gasket. The heights of the Sierpinski sub-gaskets are $11 \mathrm{~mm}$, $22 \mathrm{~mm}$ and $44 \mathrm{~mm}$. The patch is fed by the coaxial cable near the apex. 
(a)

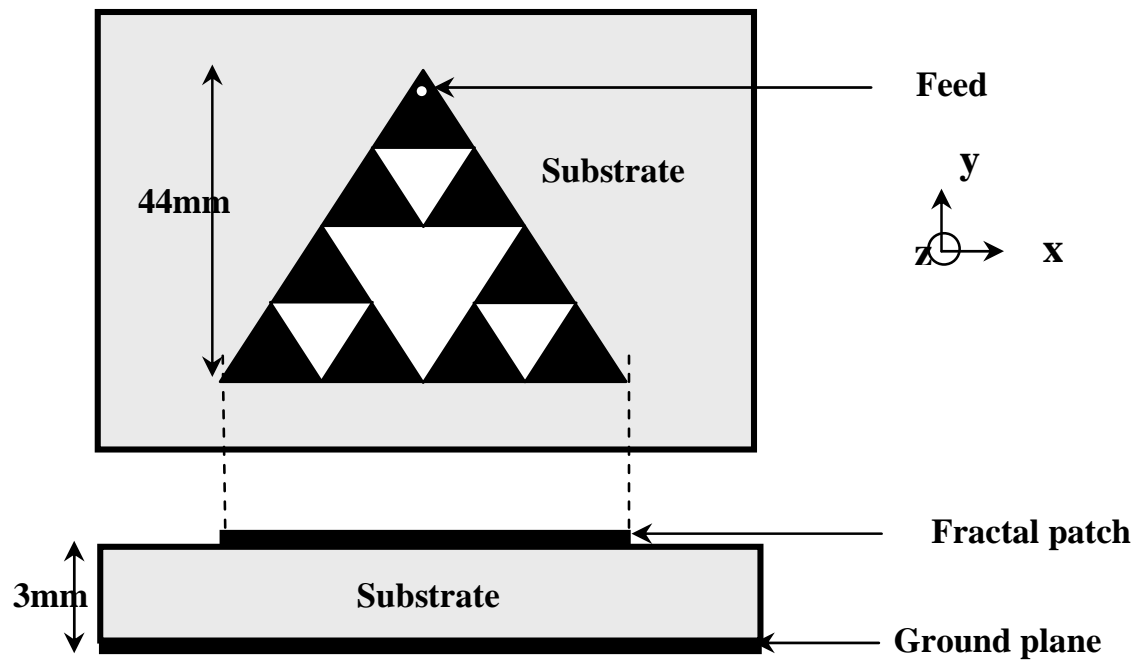

(b)

Figure 9: Top view (a) and side view (b) of the Sierpinski patch antenna.

\subsubsection{Simulation Result}

As mentioned earlier, if one neglects the center holes, an antenna performance similar to three-scaled triangular patch antenna can be expected. This is verified with the monopole Sierpinski antenna and hence we could expect the similar results here. Figure 10 shows a triangular patch antenna of length $\mathrm{L}$, where $\mathrm{L}$ is length of each sub-gasket i.e., $11 \mathrm{~mm}, 22 \mathrm{~mm}$ and $44 \mathrm{~mm}$. Triangular patches corresponding to each sub-gasket were simulated and their results are compared with the patch configuration of third order Sierpinski fractal antenna. Figure 11 (a) shows the input reflection coefficient of the triangular patch with height $44 \mathrm{~mm}$. 
(a)

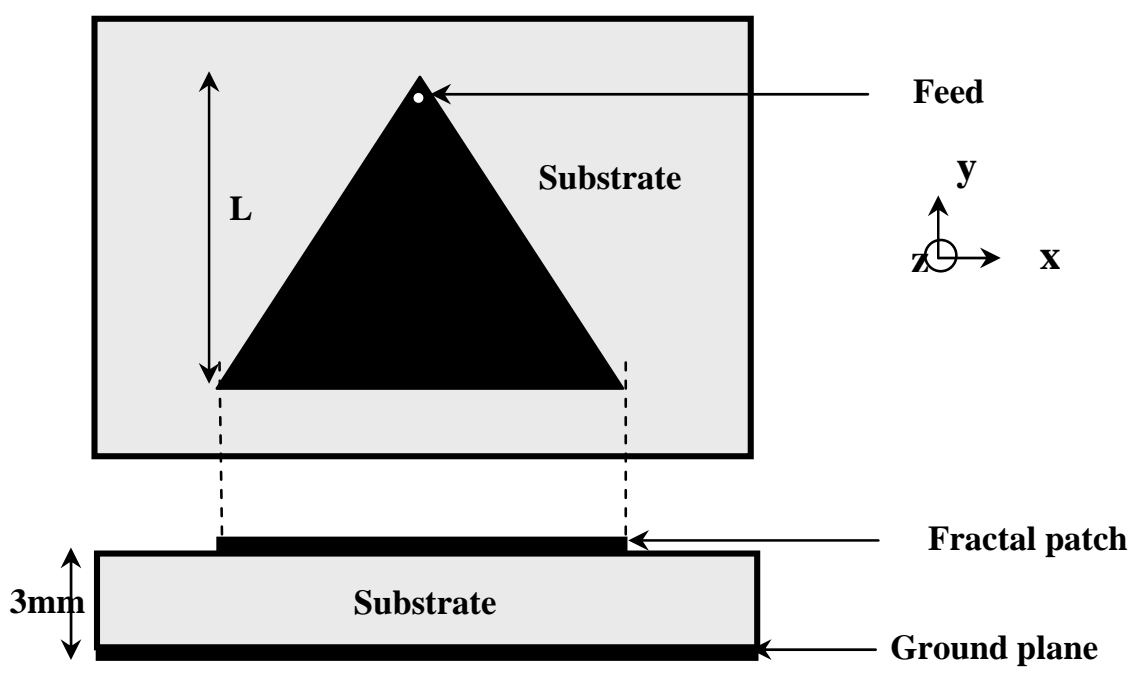

(b)

Figure10: Triangular Patch antenna of length L.

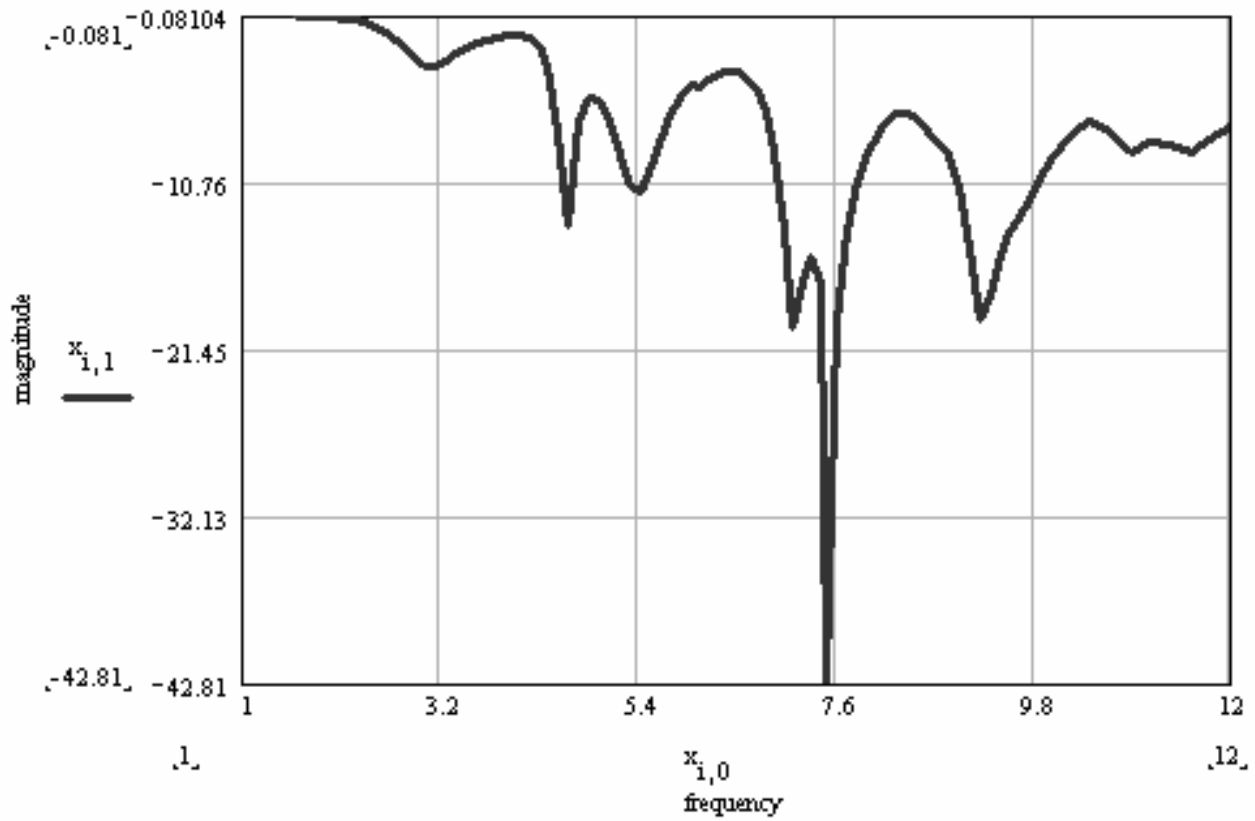

(a) 

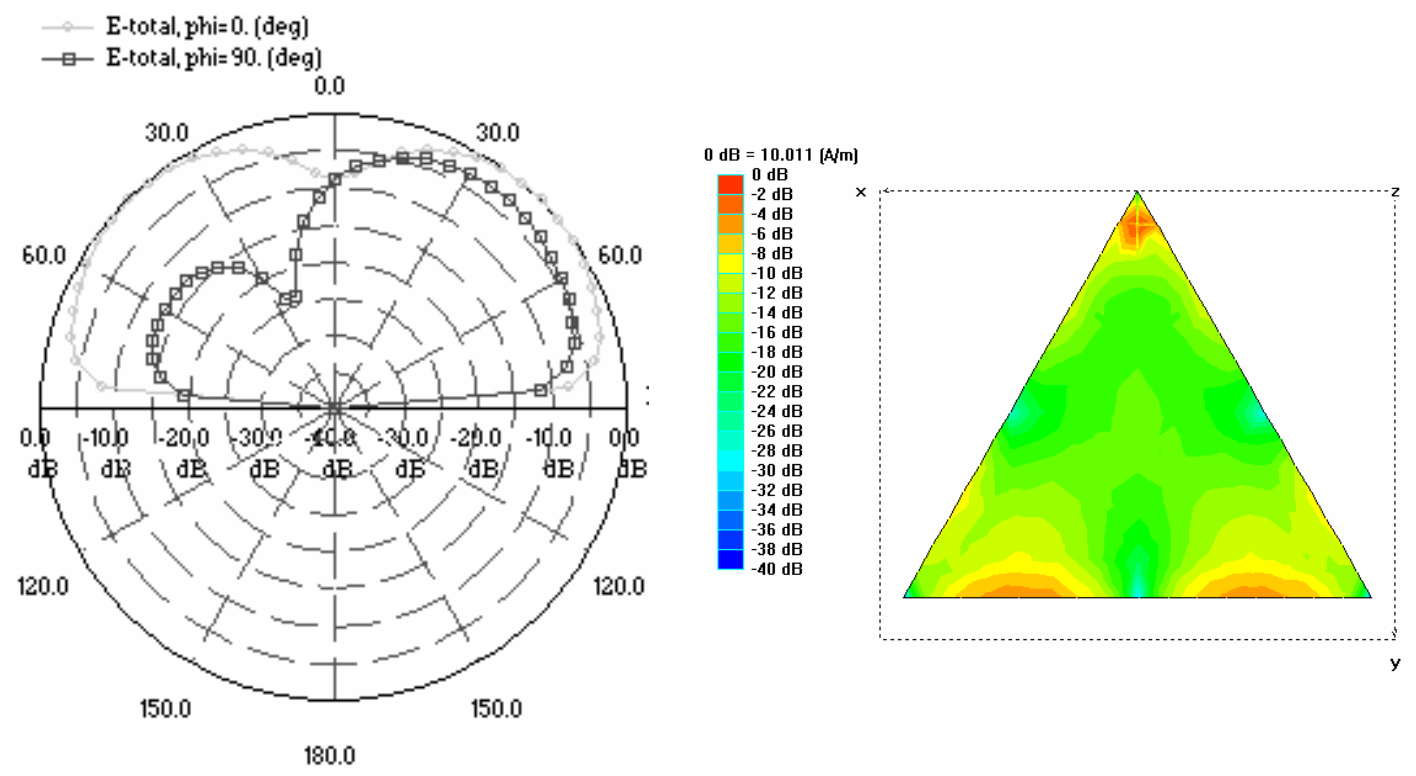

(b)

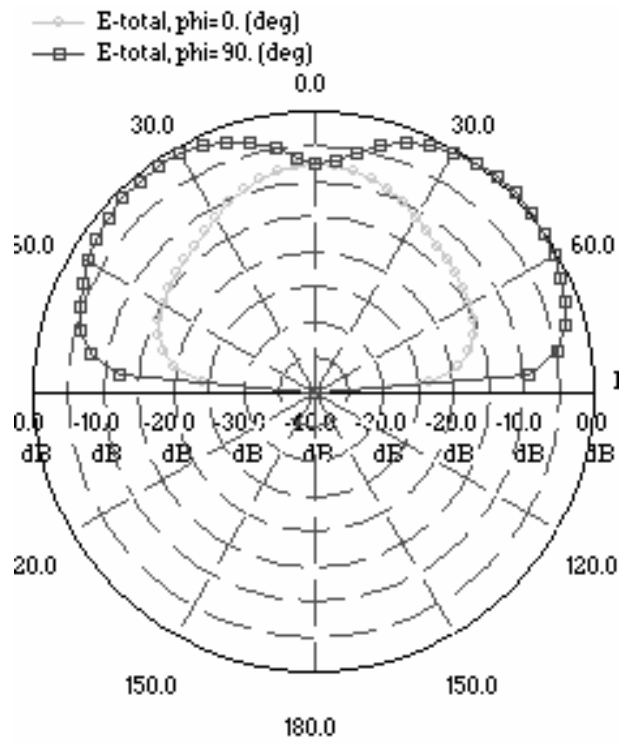

Polar Elevation Pattem

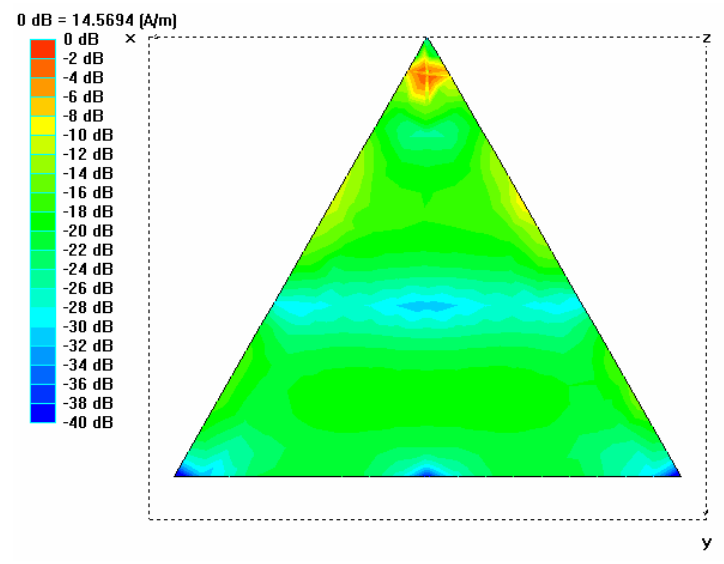

(c) 

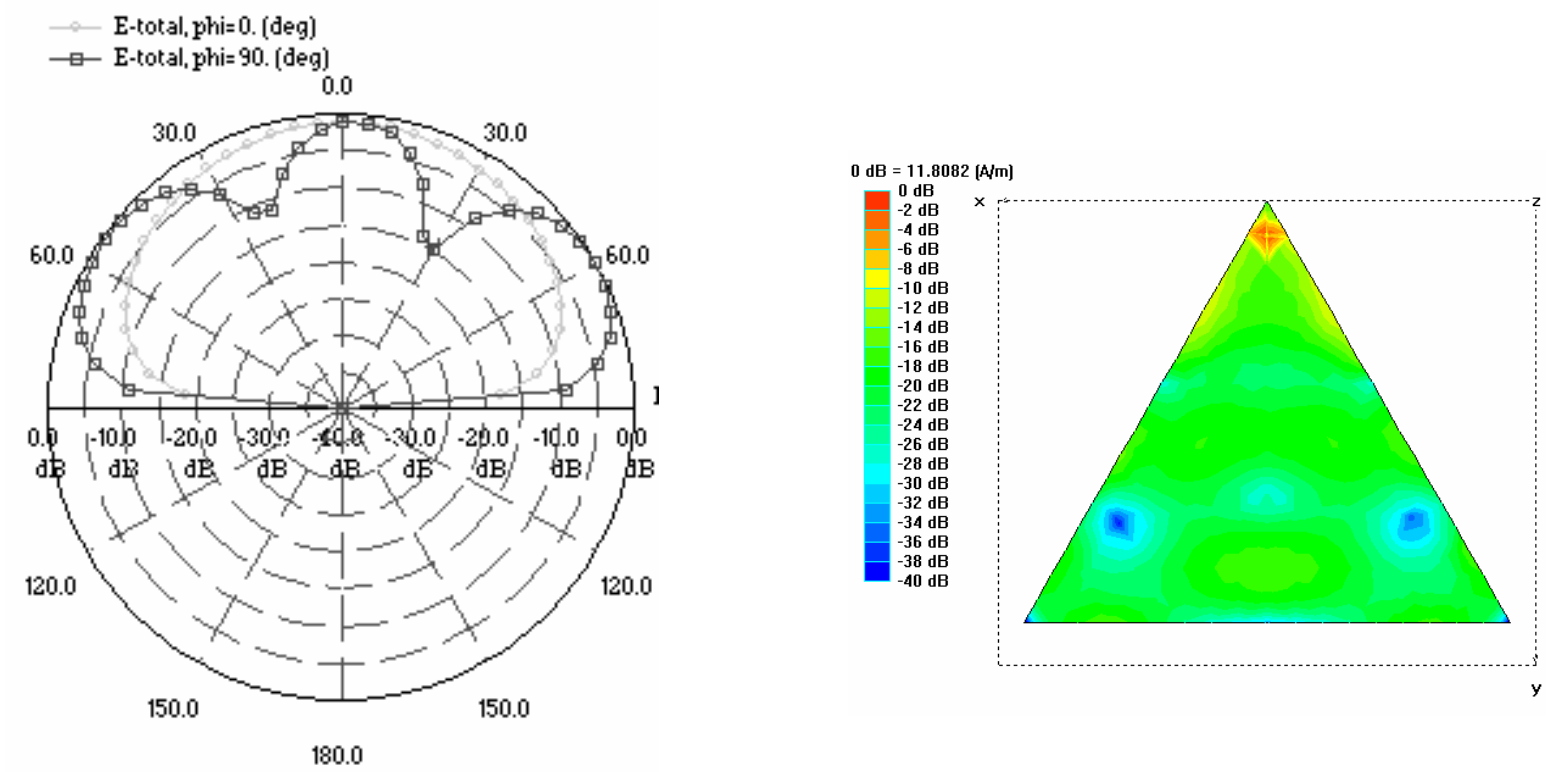

(d)

Figure 11: Characteristics of triangular patch antenna with length 44mm.(a) return loss, (b), (c), and (d) radiation pattern at $4.6 \mathrm{GHz}, 5.4 \mathrm{GHz}$ and $7.6 \mathrm{GHz}$ respectively.

Four resonances occur at 4.6, 5.4, 7.6, and $8.7 \mathrm{GHz}$ as seen in the Figure 11. The radiation pattern at $4.6 \mathrm{GHz}$ has no side lobes and main beam is perpendicular to the plane of the patch, while the radiation patterns at $5.4 \mathrm{GHz}$ and 7.6 have a notch in the main lobe. It is seen that as antenna operates at the higher resonances, it generates a radiation pattern with significant level of side lobe.

Figure 12 shows the return loss and radiation characteristics of the triangular patch antenna with height $22 \mathrm{~mm}$. There are two resonances below $12 \mathrm{GHz}: 6.2$ and $9.8 \mathrm{GHz}$. The former is due to propagation of fundamental mode where as, the latter is due to the propagation of higher modes. Radiation pattern at $6.2 \mathrm{GHz}$ has no side lobes and is fairly omni-directional main beam is perpendicular to the plane of the patch. The radiation pattern at $9.8 \mathrm{GHz}$ has a notch in the main lobe. 


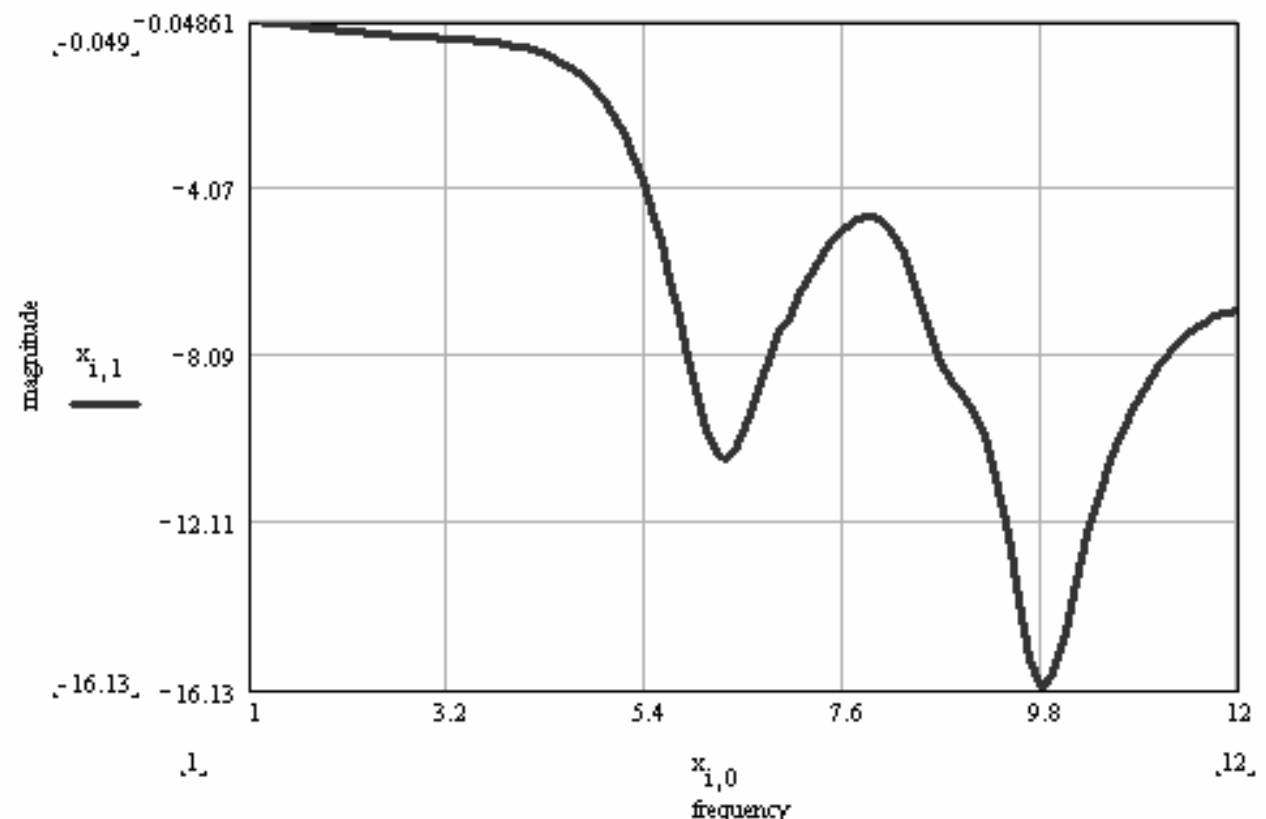

(a)
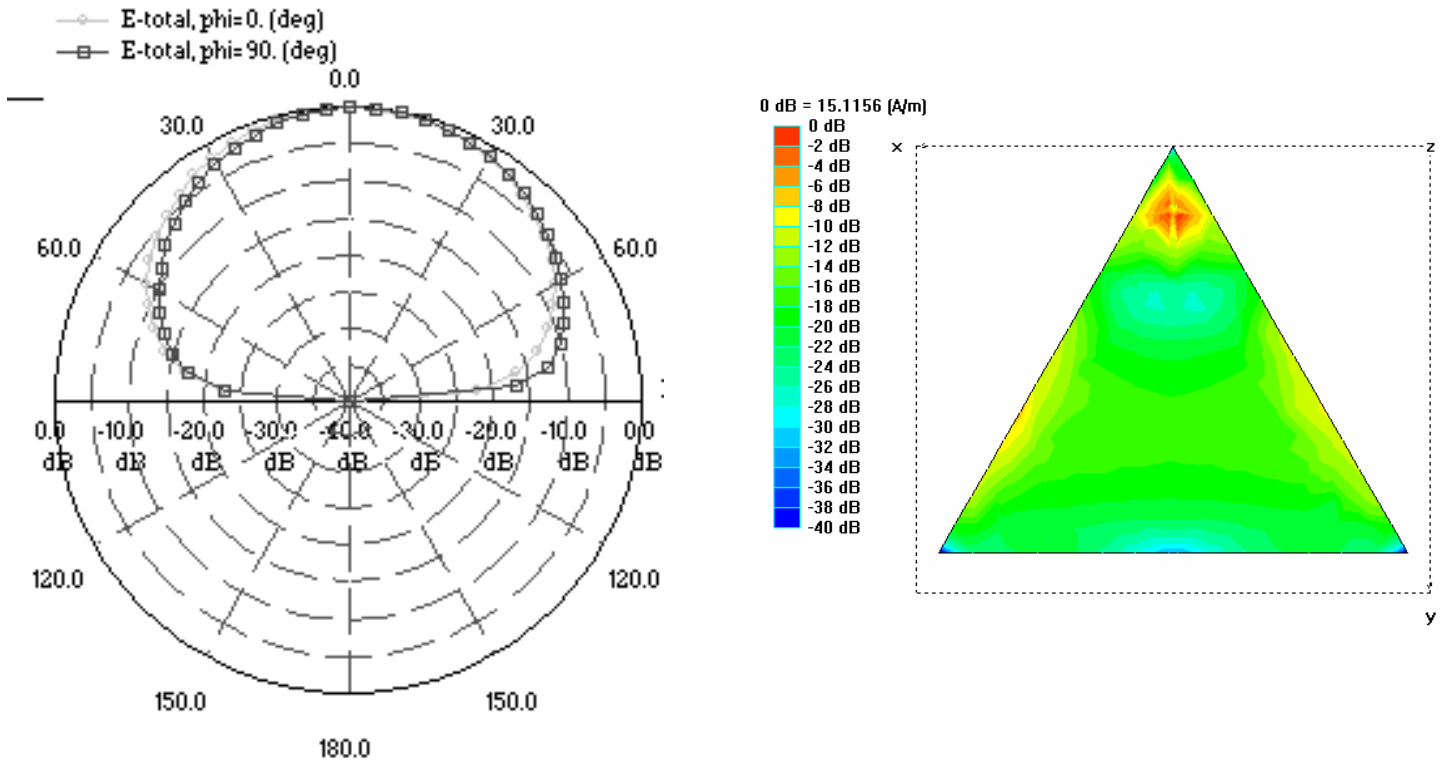

Polar Elevation Pattem

(b) 

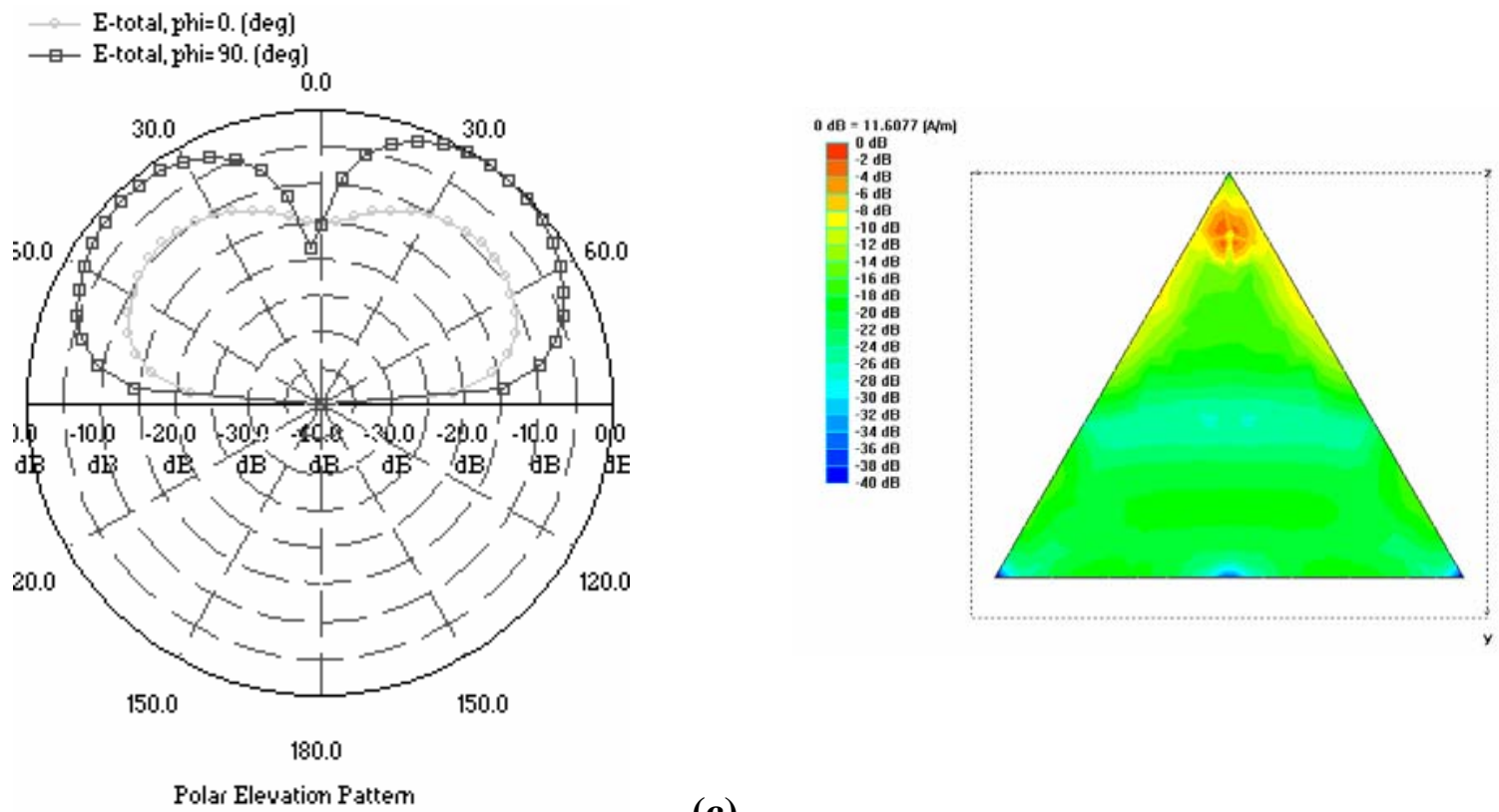

(c)

Figure12: Characteristics of triangular patch antenna with height $22 \mathrm{~mm}$. (a) return loss, (b), and (c) :Radiation pattern at $6.2 \mathrm{GHz}$, and $9.8 \mathrm{GHz}$

Figure 13 shows the simulated result of the triangular patch antenna with height $11 \mathrm{~mm}$. One resonance is seen at $10.2 \mathrm{GHz}$. The radiation pattern is omni-directional with one main lobe perpendicular to the plane of the patch. 


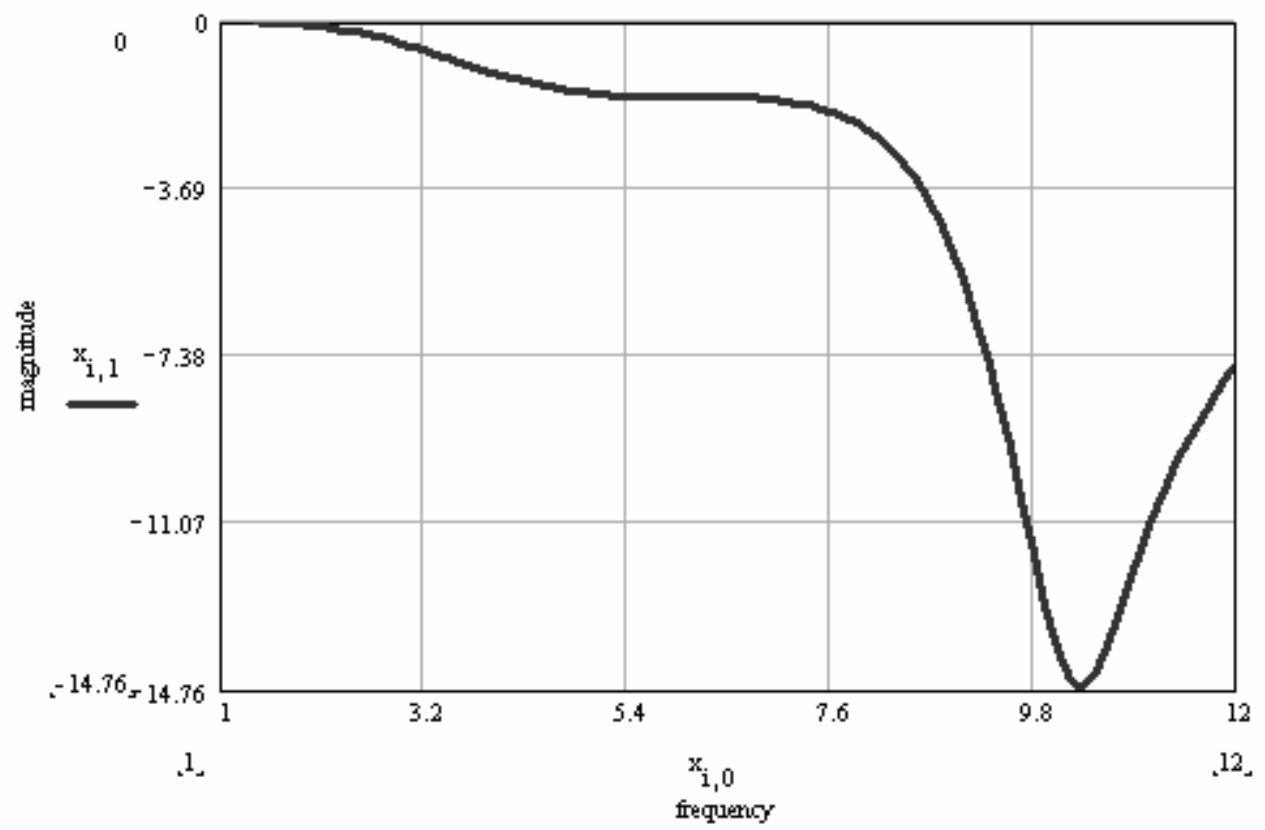

(a)
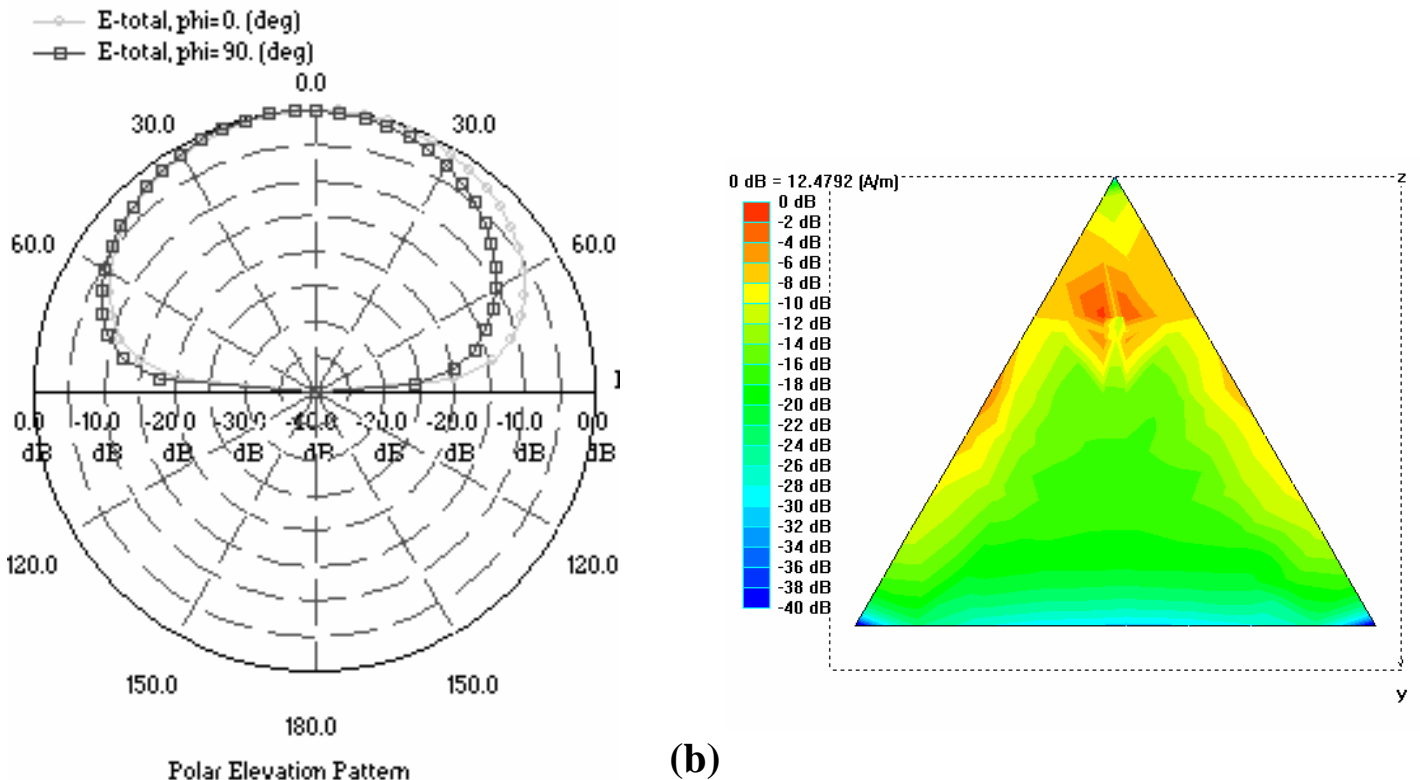

(b)

Figure 13: Characteristics of triangular patch antenna with height $11 \mathrm{~mm}$. (a) return loss, (b) radiation pattern and current distribution at $10.2 \mathrm{GHz}$. 
We would expect the characteristics of fractal Sierpinski patch antenna to inherit the behavior of the three scaled triangular patch antennas discussed above as seen in Sierpinski monopole antenna. We see that the radiation characteristics of the three triangular patch antennas are similar; however this similarity is at different frequency bands. Since the scale factor between the triangles is 2 , the radiation characteristics are similar in frequency bands that differ by a factor of two. Figure 14 shows the simulated result of the Sierpinski patch antenna. It has one resonance at $10.3 \mathrm{GHz}$ instead of three resonances corresponding to three sub-gaskets as was seen in Sierpinski monopole antenna. It shows that patch fails to provide the multi-band property as the monopole does. The radiation characteristics do not scale with frequency. In fact, the only resonance it has is at the highest resonating frequency of the monopole of the same dimension.

The reason for this is because coupling between the triangles is very weak and that current fails to adequately penetrate into the upper triangles as seen in Figure14 (b). Only smallest triangle triangular patch near the apex resonates effectively. Figure 15 shows the current distribution at the expected resonances at 3 and at $6.3 \mathrm{GHz}$. For effective radiation at certain frequency, the current should concentrate over the region of the structure comparable to the corresponding wavelength, which does not happen due to the weak coupling between the triangular patches.

Simulations done in Section 2.4 on Sierpinski monopole antenna provide an insight into the multi-band behavior of the fractal antenna. In Section 2.5 the same set of simulations are performed on the Sierpinski patch antenna with exactly same dimension. It was seen that Sierpinski patch antenna does not posses the multi-band property and study on current distribution on the patch gave a clear picture on the cause of the failure. Improving the coupling between the triangular patches could help in obtaining the desired behavior from Sierpinski patch 
antenna. In the next Chapter, we present some measures taken to improve the coupling that are found in the literature and propose new from the literature and present some designs to improve the radiation properties.

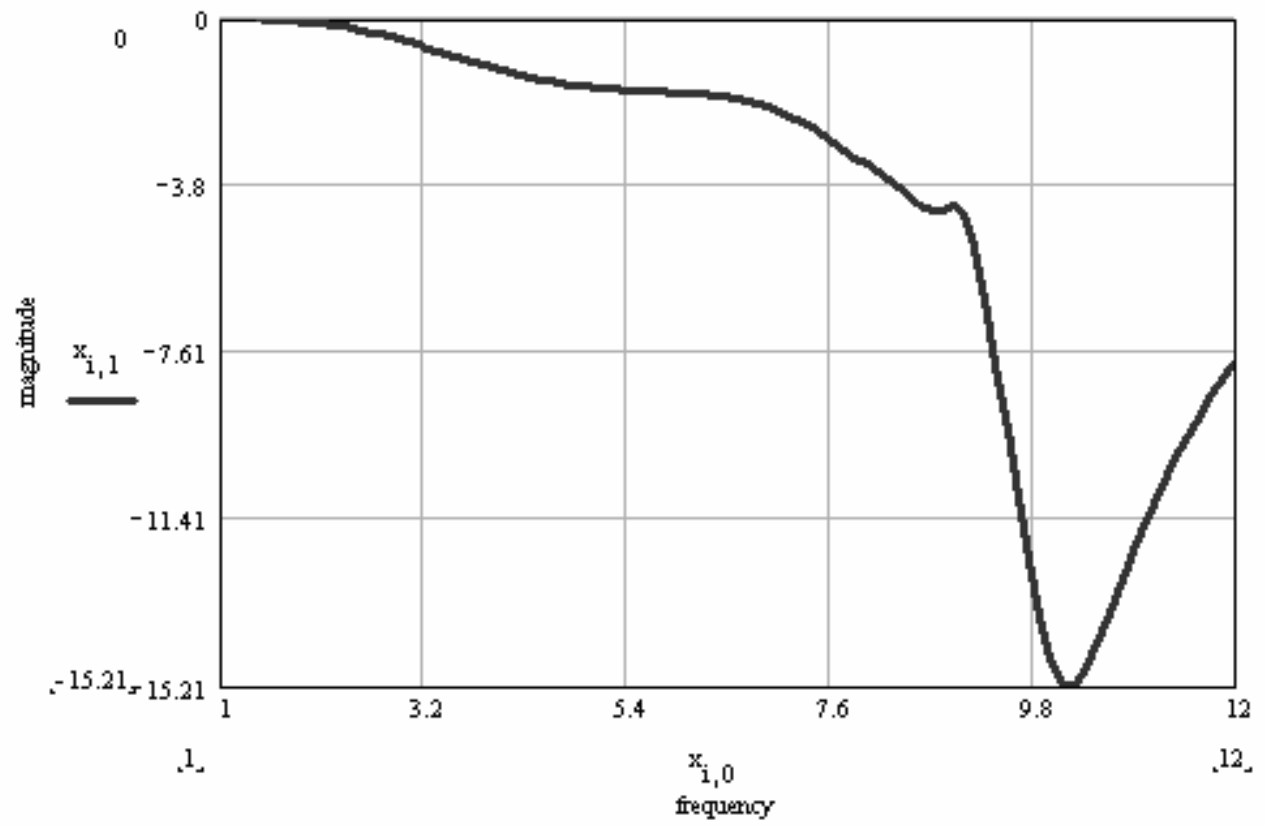

(a)

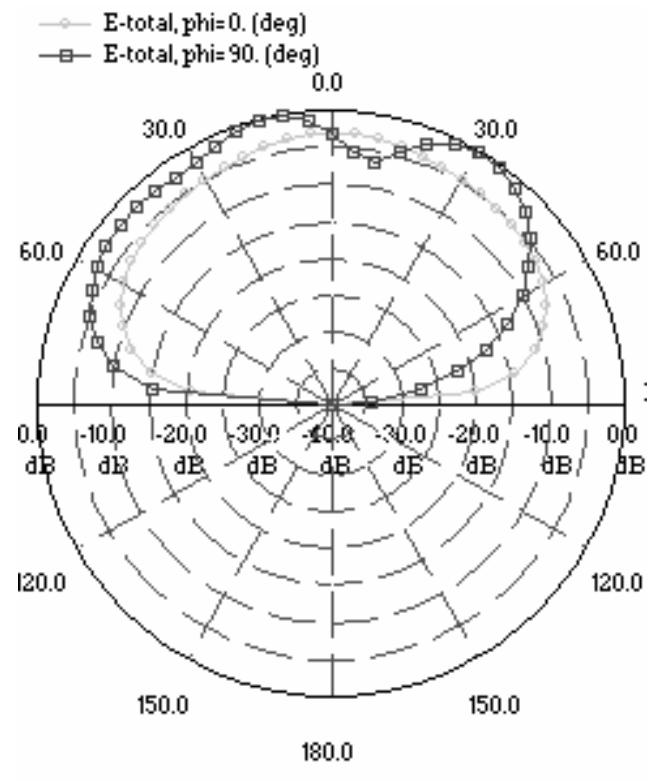

Polar Elevation Pattem

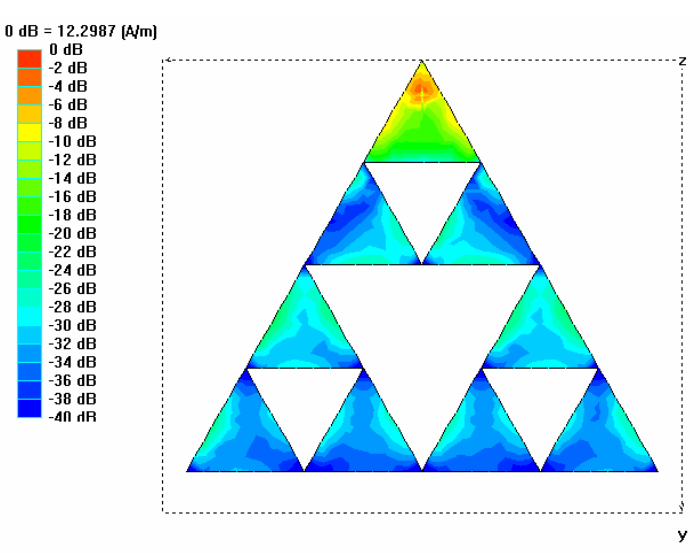

(b)

Figure 14: Characteristics of Sierpinski fractal antenna. (a) Return loss, (b) radiation pattern at $10.3 \mathrm{GHz}$. 

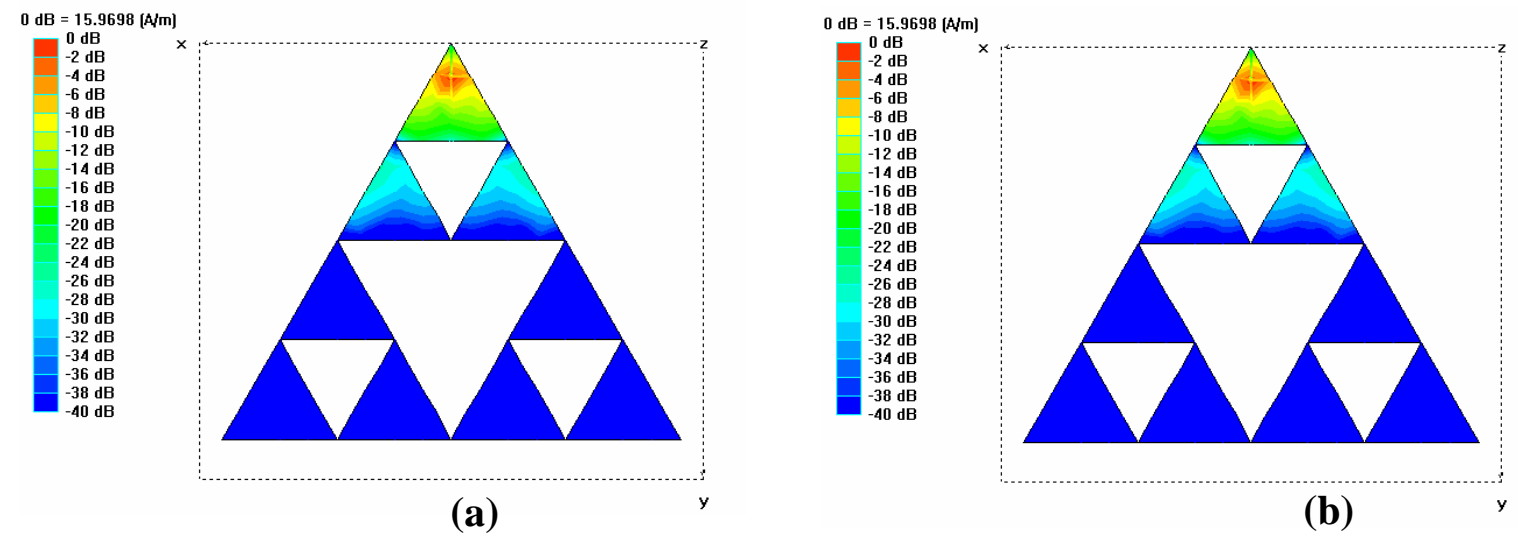

Figure 15: Current distributions at (a) $3.4 \mathrm{GHz} \&$ (b) $6.1 \mathrm{GHz}$. 


\section{CHAPTER THREE: STACKED FRACTAL ANTENNA DESIGN}

\section{$\underline{3.1 \text { Recent Developments on Sierpinski Patch Antenna }}$}

Several approaches to realize the multi-band behavior of the Sierpinski patch antenna have been proposed [17-21]. A two-layer configuration as shown in Figure 3.1 [21] had the best performance and its results are duplicated in this section. A top grid layer of width $0.7 \mathrm{~mm}$ as shown in Figure 16(a) is placed above the original Sierpinski patch on a substrate whose thickness is $0.127 \mathrm{~mm}$ and dielectric constant is 2.2 to provide stronger electromagnetic coupling between triangular patches.

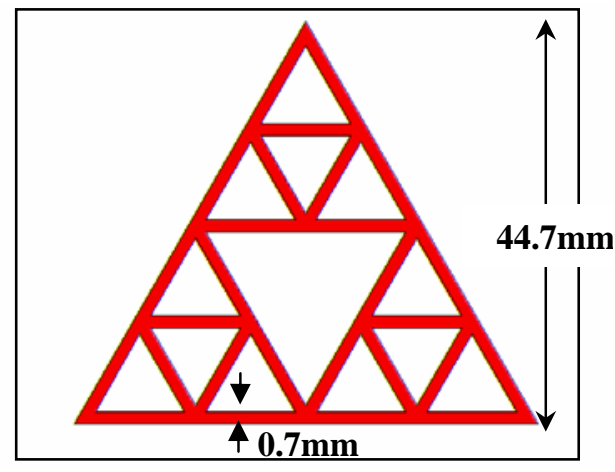

(a)

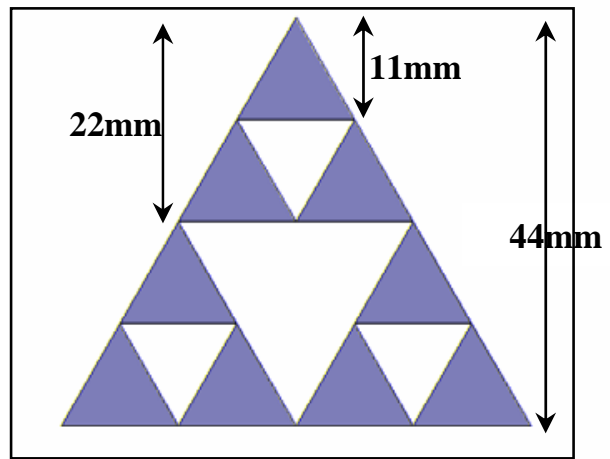

(b)

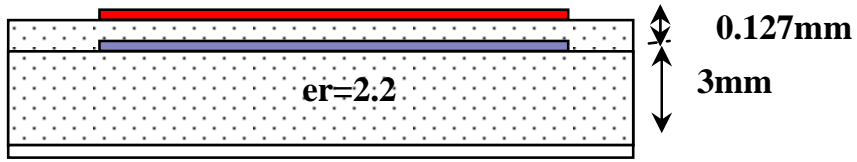

(c)

Figure 16: Sierpinski patch antenna with grid (a) grid, (b) original fractal patch, (c) crosssectional view of the structure. 
The insertion loss of Sierpinski patch antenna with grid on the top layer is plotted in Figure 17. As can be seen a total of four resonances are seen at 5, 7, 9.1, 11.3 GHz. The simulated results are comparable to those obtained in the literature [21].

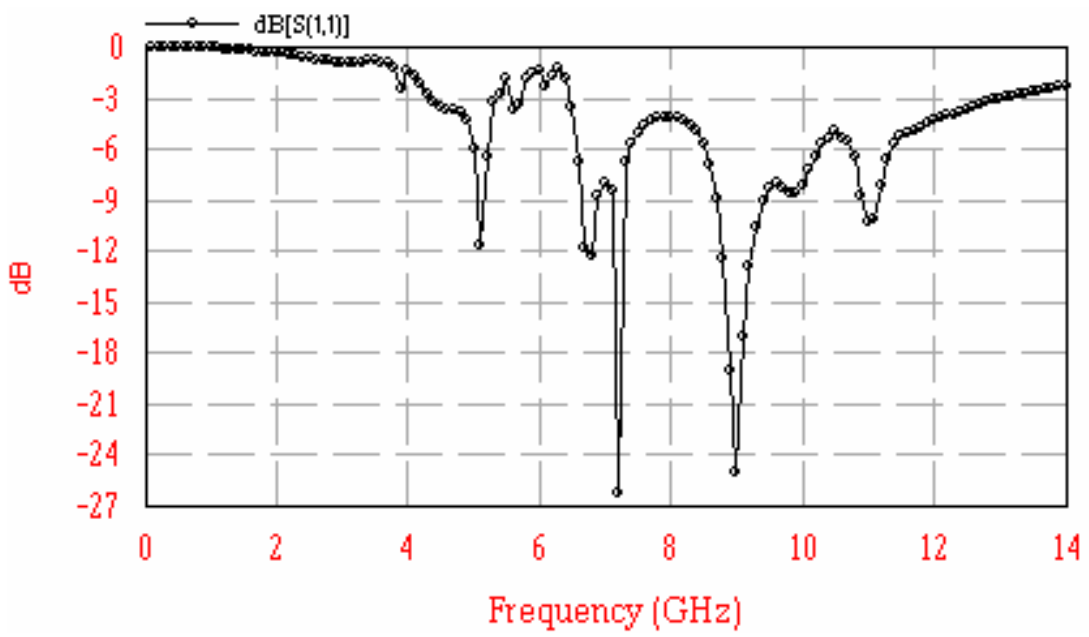

Figure 17: Return loss of Sierpinski patch antenna with grid.

Modifications were done on the above patch antenna to get a better result [23]. To improve the matching characteristics at the low frequency bands, the top edges were truncated and shorting pins are placed at certain locations (Figure 18). The edge truncation alters the electrical length of the triangular antenna and hence lowers the first resonating frequency with better impedance matching. Shorting pins placed between bottom layer and the ground layer, were three different locations helps to further improve the impedance at resonances and radiation pattern. 


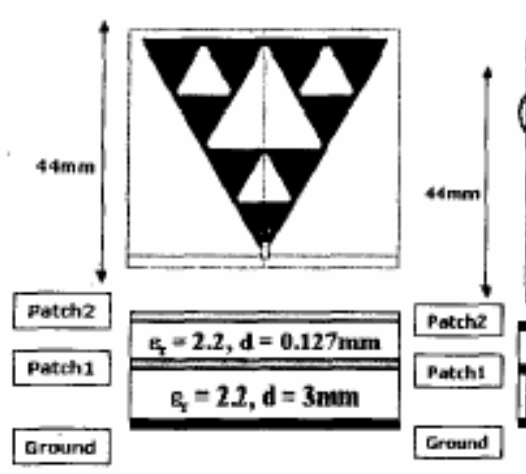

(a) Original

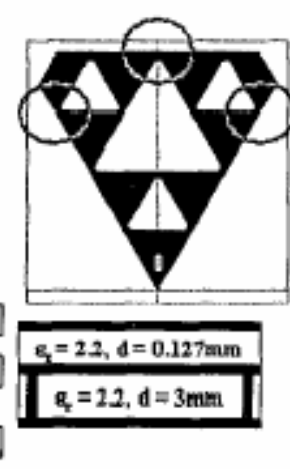

(b) Modified

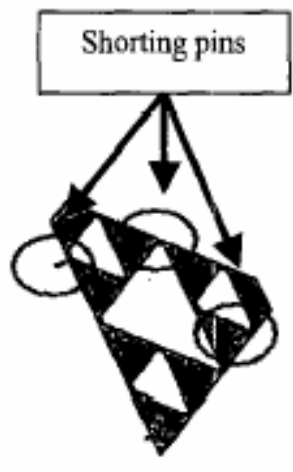

(c) 3D view

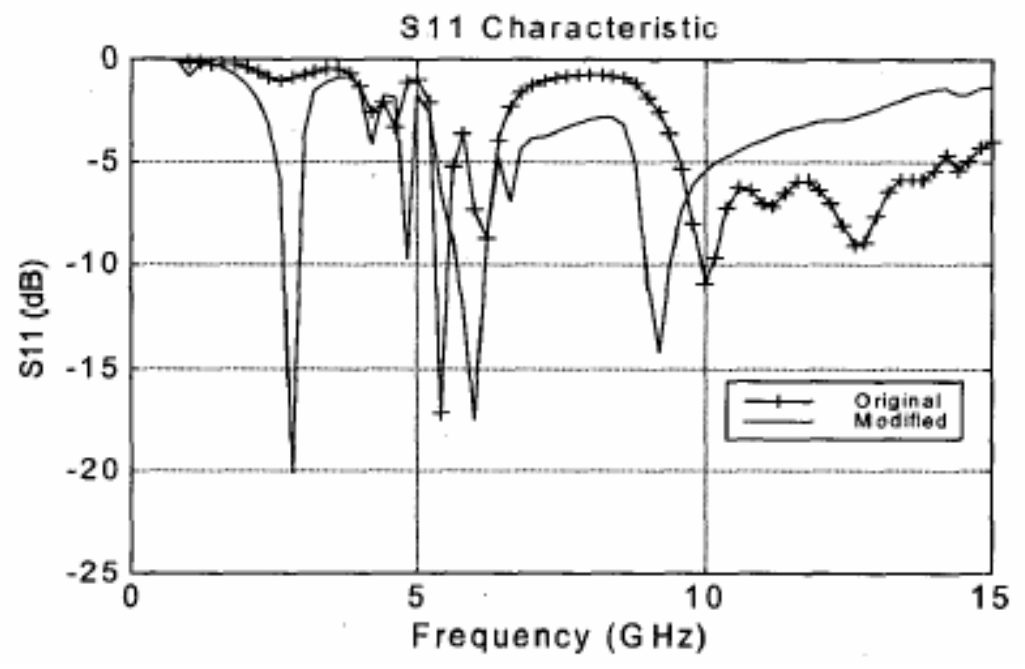

Figure 18: Geometry and S11 characteristics of the modified patch antenna [23].

A Sierpinski grid antenna which is just a grid version of the Sierpinski gasket has been studied in [18]. This is also trimmed and shorted at different points to enhance the impedance matching at resonating frequencies. The structure of the grid antenna is shown in Figure 19. 

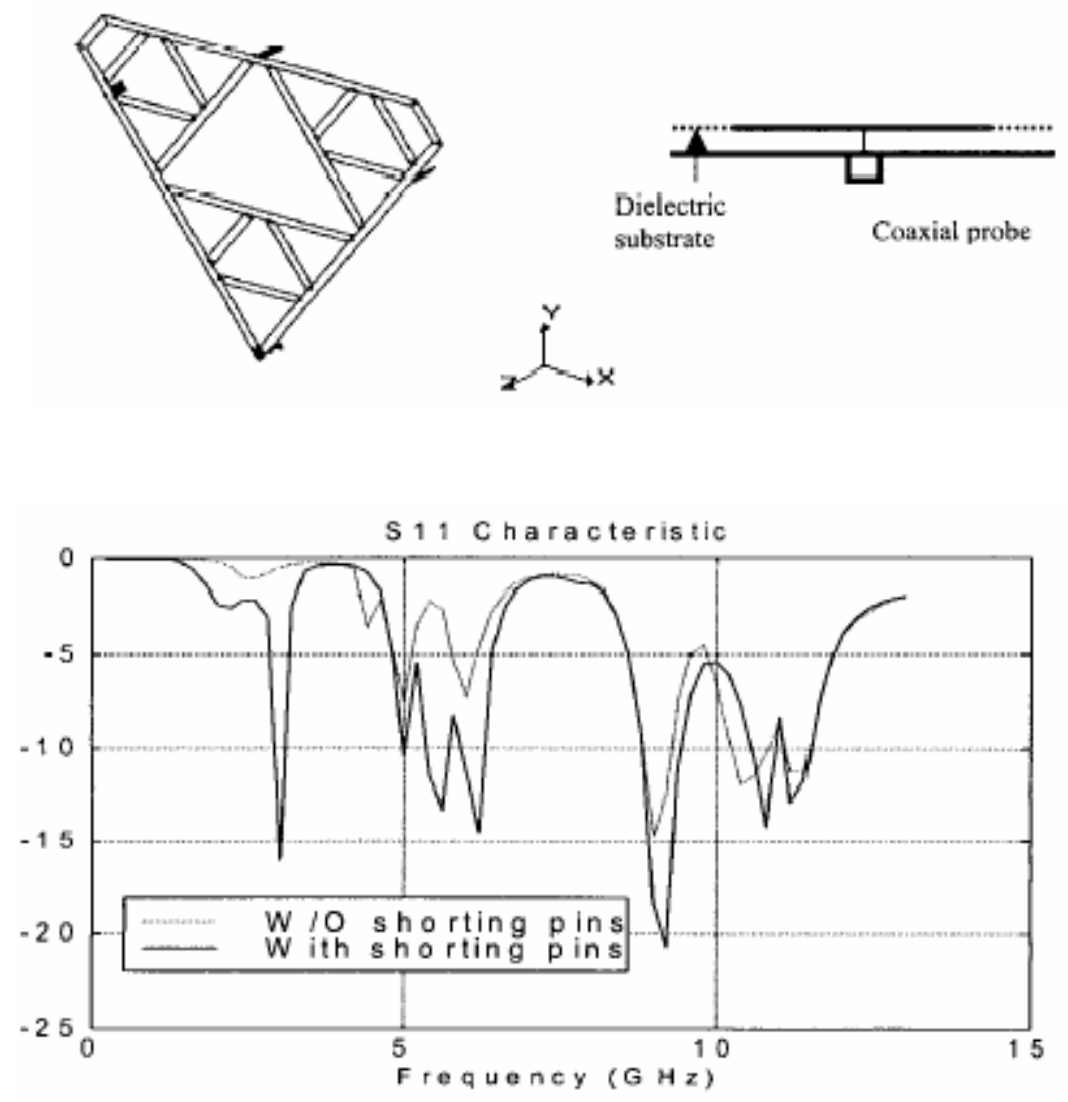

Figure 19: Geometry and S11 characteristics of modified Sierpinski grid antenna [18].

\subsection{Design of the Stacked Sierpinski Antenna}

The main objective of this research is to explore and improve the multi-band properties of the fractal antenna in a patch configuration. Modifications were made on second order patch antenna to simplify the complexity. The design procedure and simulation steps, conducted using IE3D software package, are discussed in detail in this Chapter.

The entire design is carried out in four stages.

1. Merging of top layer grid to the fractal.

2. Using a Stacked fractal design to obtain more resonances.

3. Truncation of corners to get better match. 
4. Set up of a gap coupled structure to improve the radiation pattern.

\section{$\underline{\text { 3.3 Fractal Sierpinski Merged With Grid }}$}

It has been discussed in Chapter 2 that the Sierpinski fractal antenna in patch configuration doesn't perform as good as the same fractal antenna in monopole configuration. It was shown that placing a grid on the top of the Sierpinski fractal layer helped to make patch configuration of Sierpinski fractal antenna work at multi-frequencies. To simplify the structure and make it a single layer structure, the top grid layer could be merged with the fractal layer. At the same time it is crucial to make sure that the important radiation properties of the two layer structure are maintained. Here merged fractal structure is simulated and the result is compared with the two layer structure of the same dimension.

The original second order Sierpinski fractal antenna is shown in Figure 20(a). The height of the triangles in the subgasket are $44 \mathrm{~mm}$ and $22 \mathrm{~mm}$. A grid layout corresponding to this structure is shown in Figure 20(b) and the Figure 20(c) shows the sideview of the new structure that is formed by merging the fractal and grid. This modified second order fractal antenna is constructed on a substrate with dielectric constant 2.2, with a height of $3 \mathrm{~mm}$. All the structures in this Chapter have the same antenna conguration and substrate parameter unless otherwise specified.The antenna is fed by a coaxial cable at the apex (top) and is simulated using IE3D. 


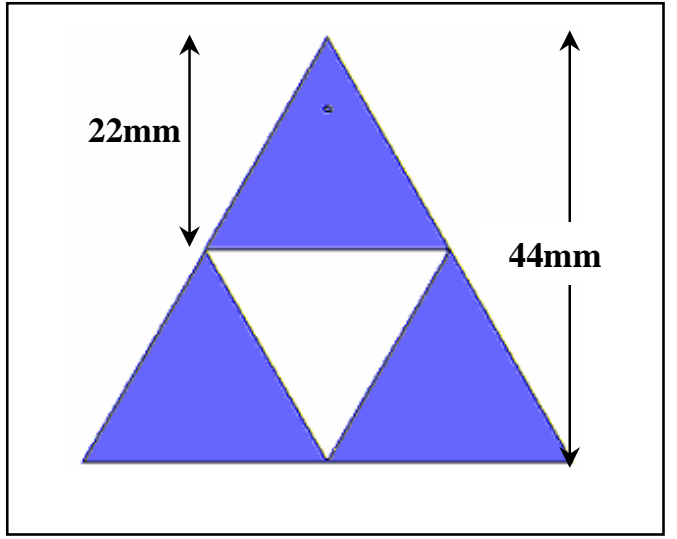

(a)

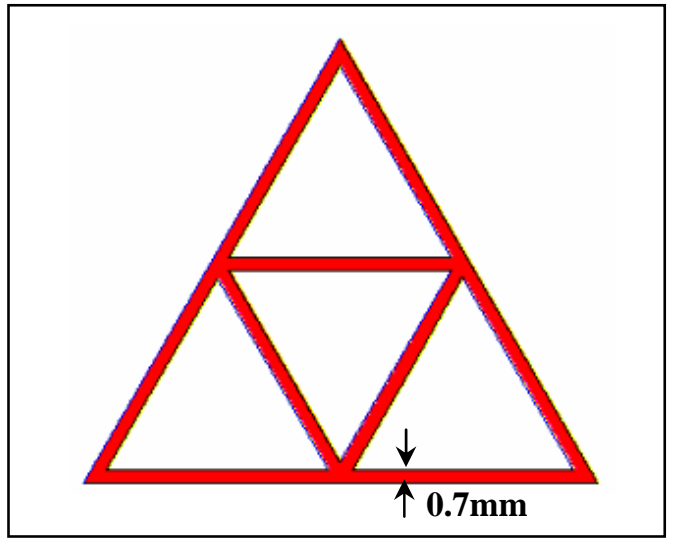

(b)

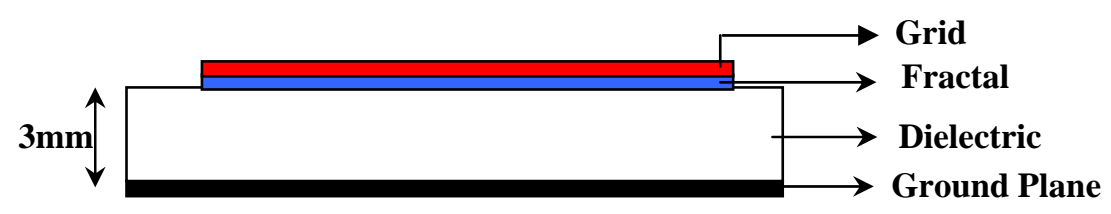

(d)

Figure 20: (a) Sierpinski fractal, (b) grid structure, and (c) side view of the overlapped fractal antenna

\subsubsection{Simulation Results}

The simulation result of the modified Sierpinski fractal antenna that is formed by merging the top layer grid and the middle layer fractal is shown in Figure21. Figure 17(a) shows the return loss of the antenna. It shows three resonances at 7 between 1 and $9 \mathrm{GHz} 5.2,6.2$ and 8 GHz. The current distributions and radiation patterns are shown in Figure 21 (b), (c), and (d). 


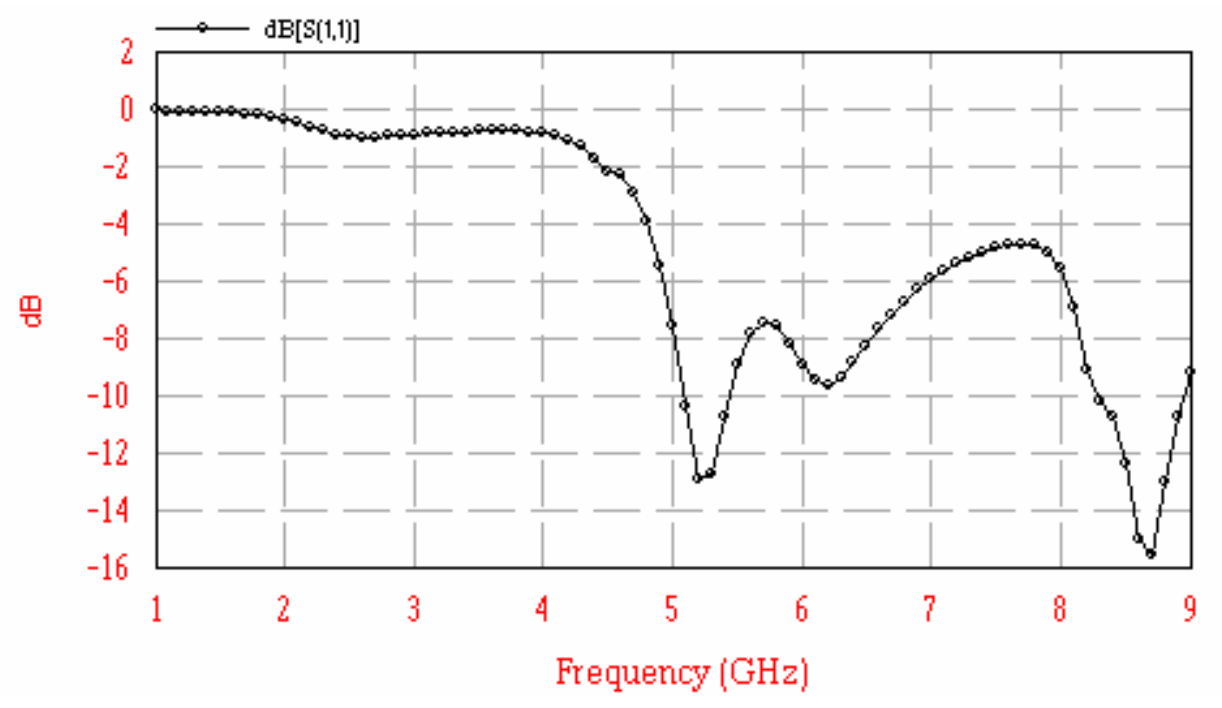

(a)
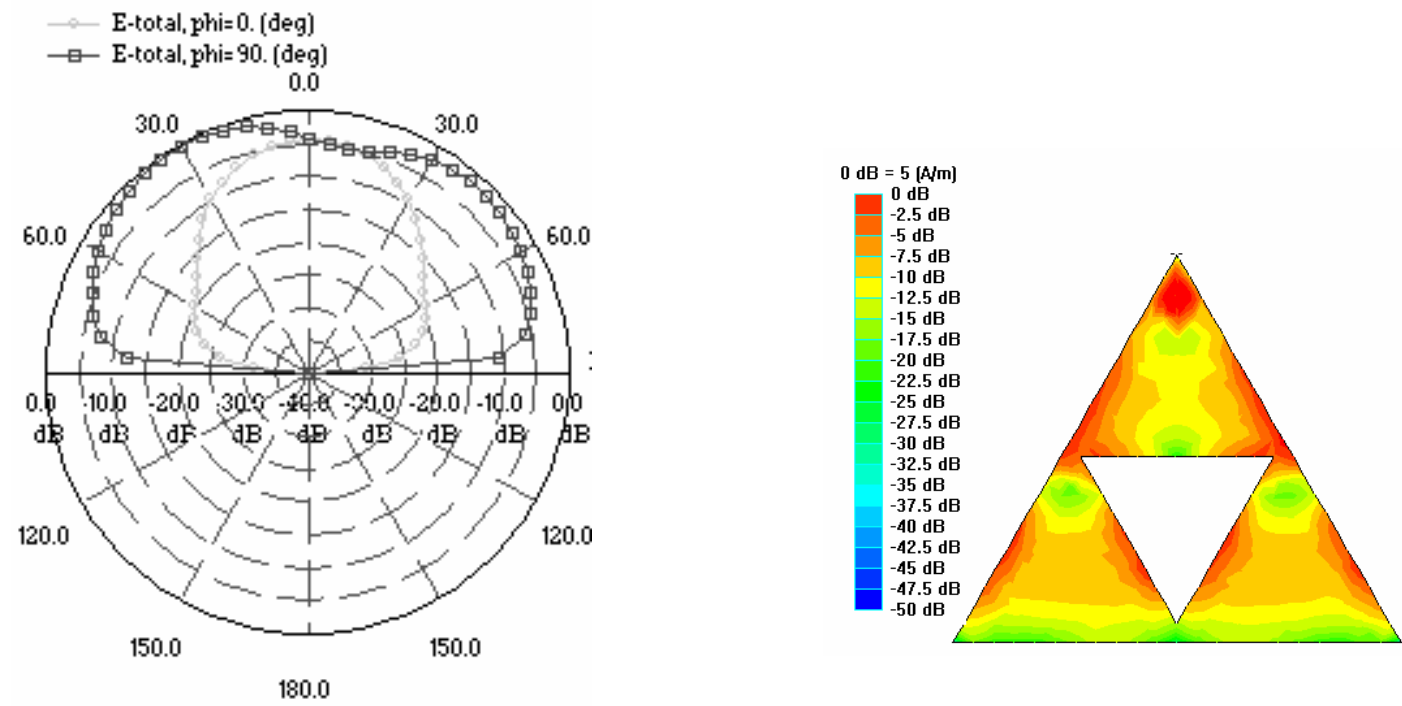

Polar Elevation Pattem

(b) 


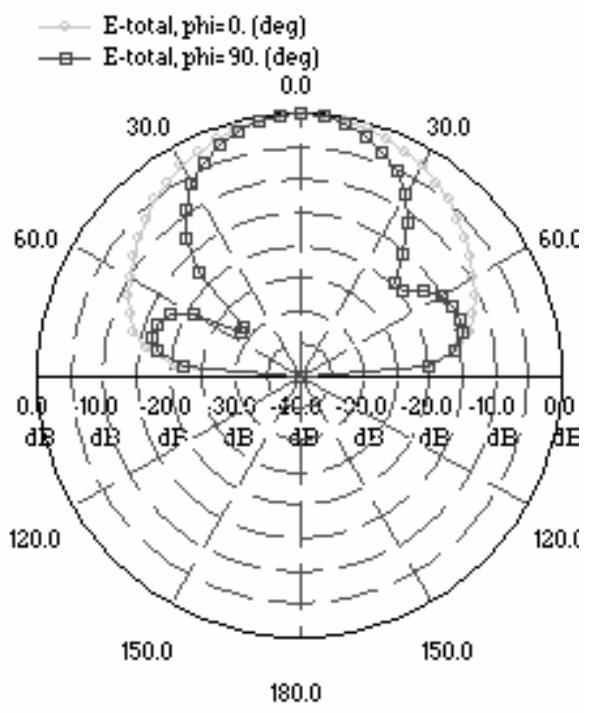

Polar Elevation Pattem

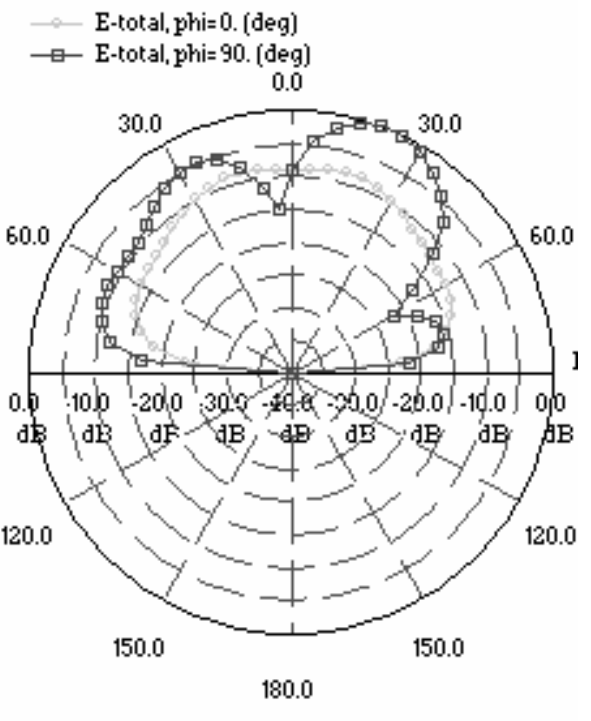

Polar Elevation Pattem

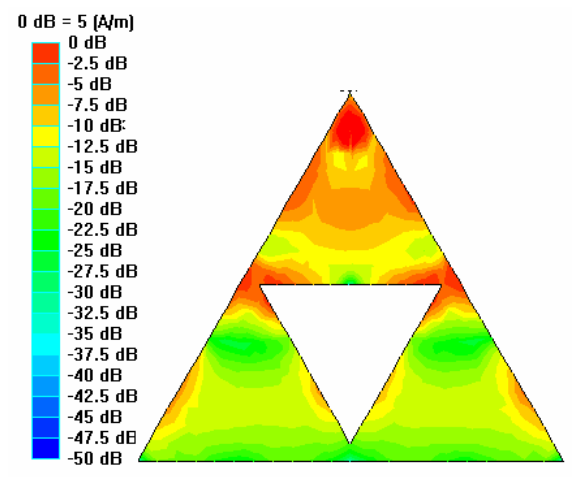

(c)

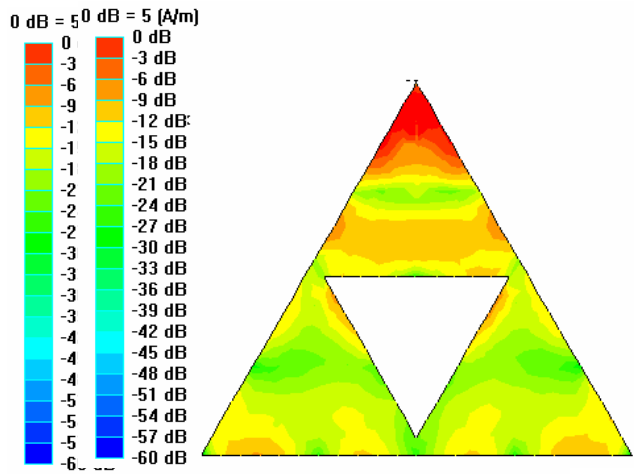

(d)

Figure 21: Characteristics of the merged Sierpinski fractal antenna. (a) Return loss, Radiation patterns and current distributions at (b) $5.2 \mathrm{GHz}$, (c) $6.2 \mathrm{GHz}$ and (d) $8.7 \mathrm{GHz}$. 


\subsubsection{Comparison of Result}

The behavior of the two layered second order Sierpinski fractal grid antenna (with the grid on the top) was compared with that of the modified Sierpinski antenna with Sierpinski fractal and grid merged. The results for the return loss are shown in Figure 22(a). A slight difference in the resonance frequency seen at high frequency can be due to the effect of the capacitance that is formed due two layers of the double layer structure.

The current distributions and radiation patterns at all the resonances of the two layered structure were and presented in Figure3.3 (b), (c) and (d). When these plots are compared with the corresponding plots of Figure 21 (b), (c), and (d) it is seen that radiation patterns are very similar. It can be noticed that the two layered structure provides a better current flow and hence better coupling and explain the reason why the two layered structure has better match at resonating frequencies than the modified one.

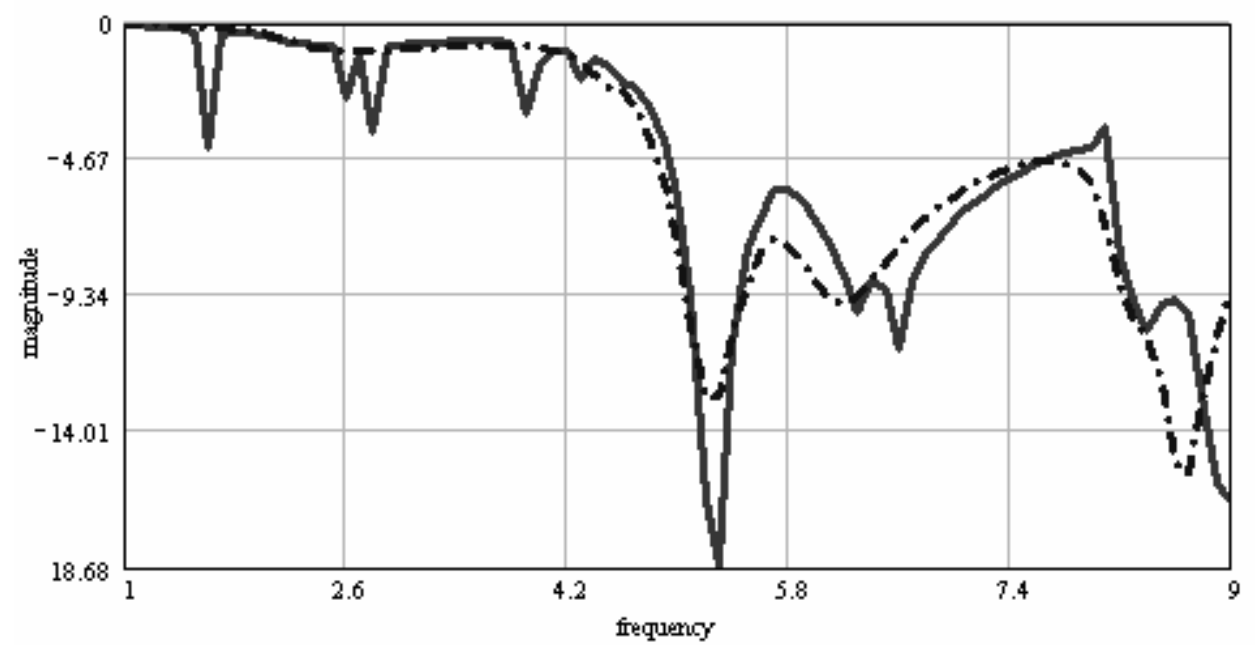

(a) 


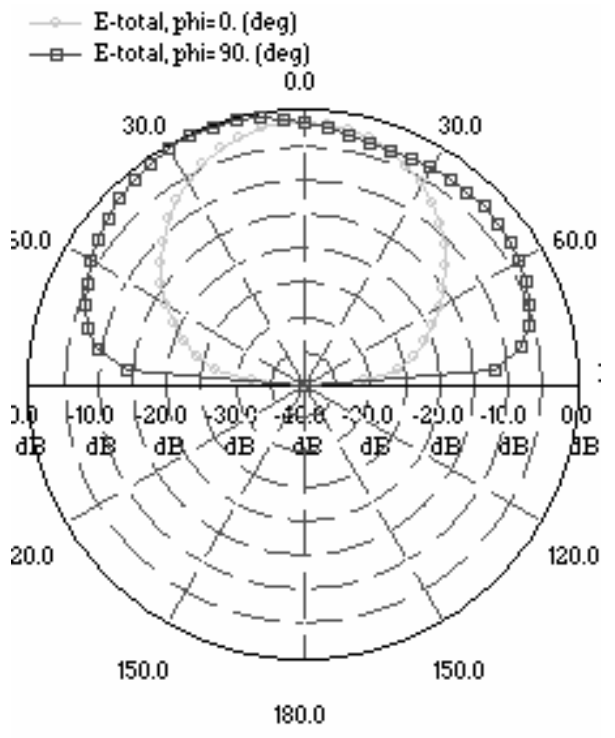

Polar Elevation Pattem

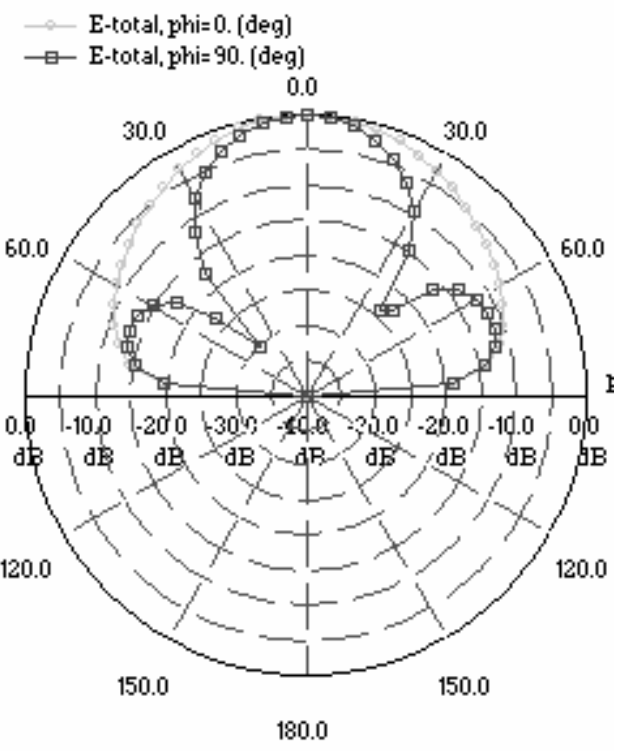

(b)
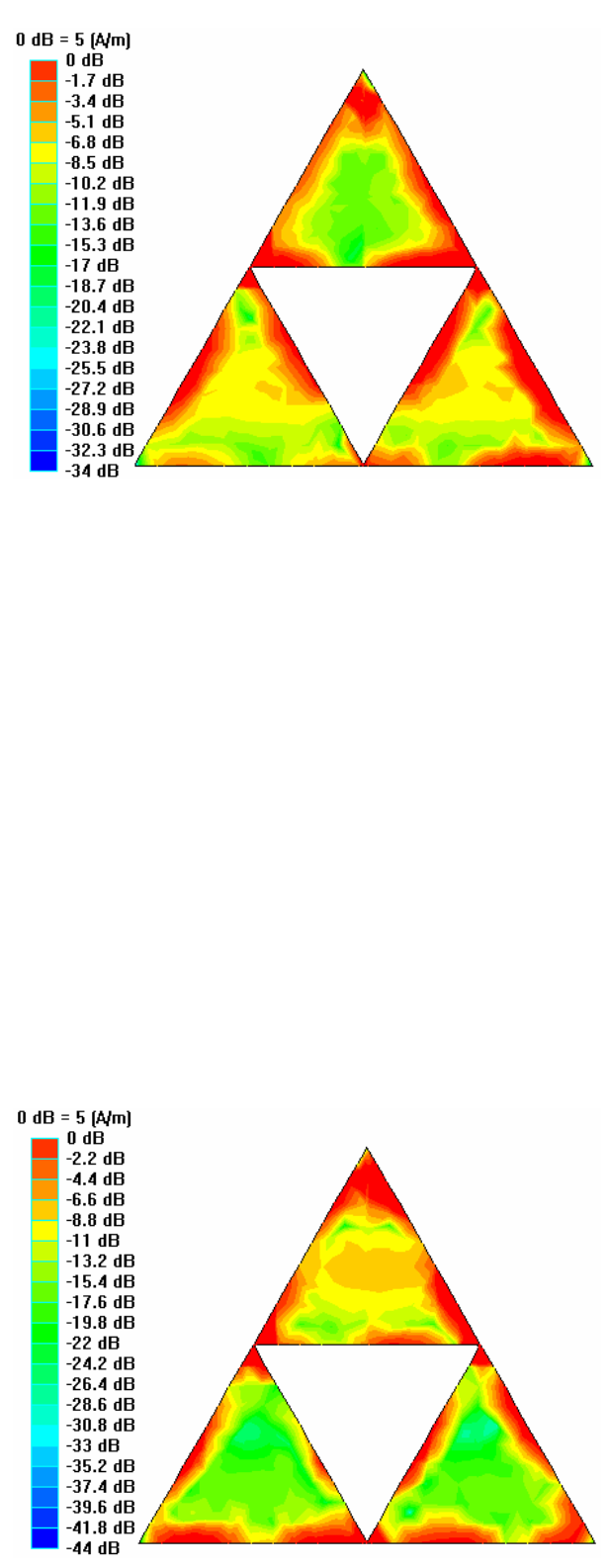

(c) 


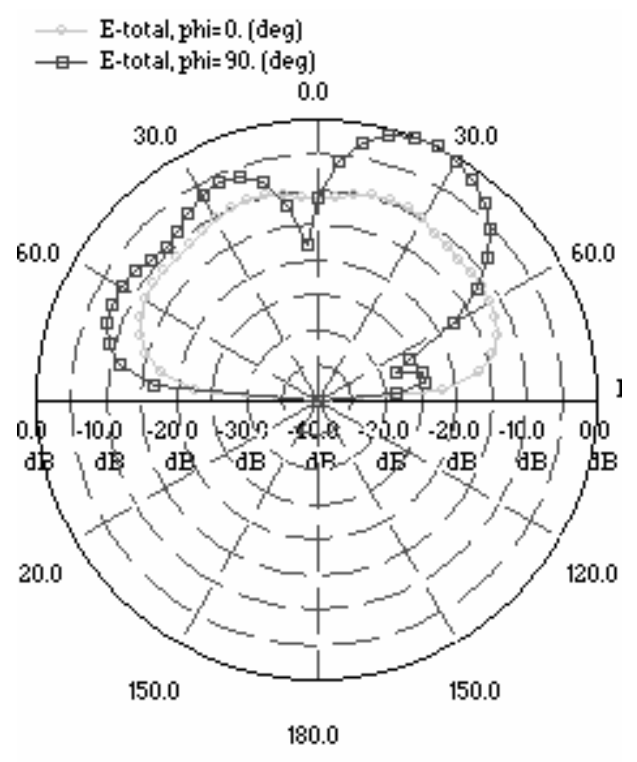

Polar Elevation Pattem

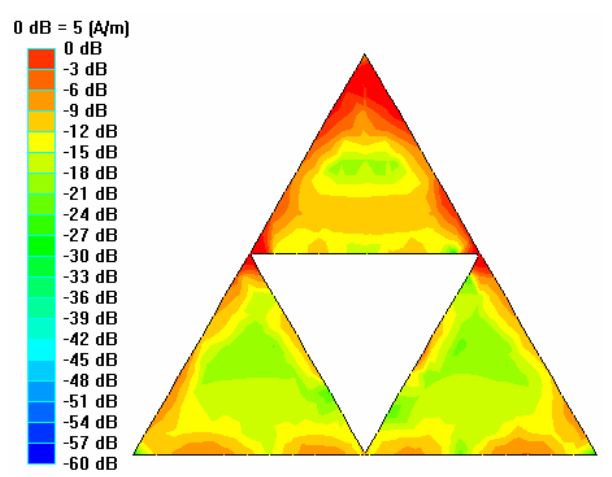

(d)

Figure 22: Characteristics of the Sierpinski fractal antenna with grid. (a) Return loss (dotted line: Sierpinski with grid, solid line: merged Structure), radiation pattern and current distribution at (b) $5.2 \mathrm{GHz}$, (c) $6.6 \mathrm{GHz}$ and (d) $9 \mathrm{GHz}$.

\section{$\underline{3.4 \text { Stacked fractal antenna }}$}

It has been shown that shown that bandwidth of a rectangular patch antenna can be increased if a rectangular section from the center of the patch is cut and moved to a lower layer, forming a double layer structure [24]. This concept is used here to obtain better multi-band characteristics.

The structure of the stacked Sierpinski fractal antenna is shown in Figure 23, Figure 23(a) shows the top view and 19(b) shows the side view of the antenna. It is a two layered structure. The top layer, shown in blue, is the modified Sierpinski fractal antenna as described in section 3.2. A triangular patch with the size same as that of the center hole of the fractal antenna is 
placed on the second bottom layer (red color). These two layers are connected using the shorting patch (black color) as shown.

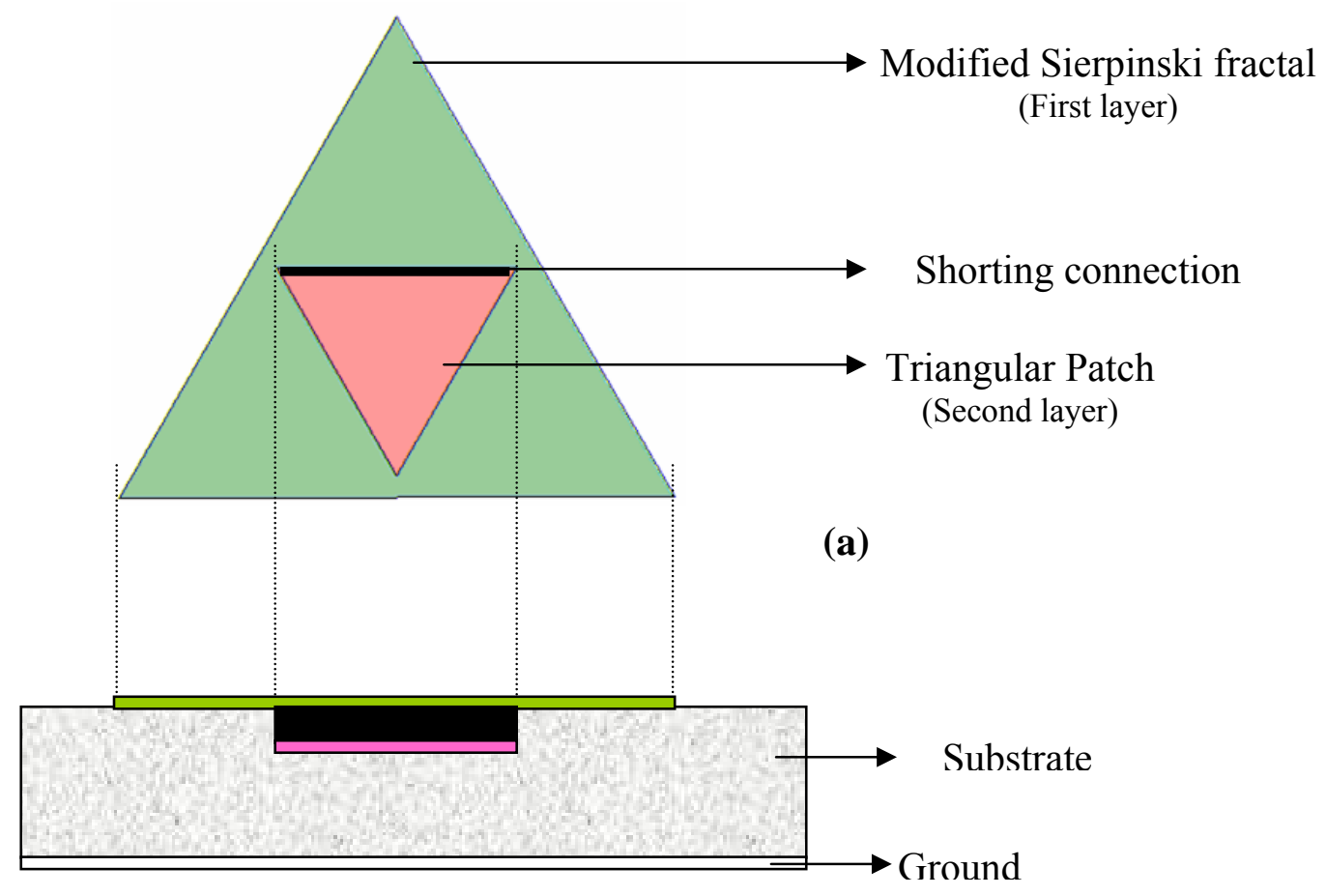

(b)

Figure23: Stacked Fractal (a) top view, and (b) side view

\subsubsection{Simulation Results}

The input return loss of the Stacked Sierpinski fractal antenna is plotted in Figure 24 (a).

Four resonances at 4.5, 5.1, 6.2 and 7.4 GHz are obtained. The Radiation patterns and current distributions at these resonance are plotted in (b), (c), (d), and (e) of Figure 24. An additional resonance at $4.5 \mathrm{GHz}$ is obtained in this stacked configuration of Sierpinski fractal antenna. It can also be observed that the return loss at the resonances of the first three resonances is not very good. This is improved by trimming the edges of the triangle as described in the following section. 
It is seen that current distribution at $4.5 \mathrm{GHz}$ is similar to the current distribution of the fundamental mode of triangular antenna. The smaller triangle added in the hole formed by the Sierpinski fractal antenna completes the triangle, causing this mode and hence its resonance to be noticed.

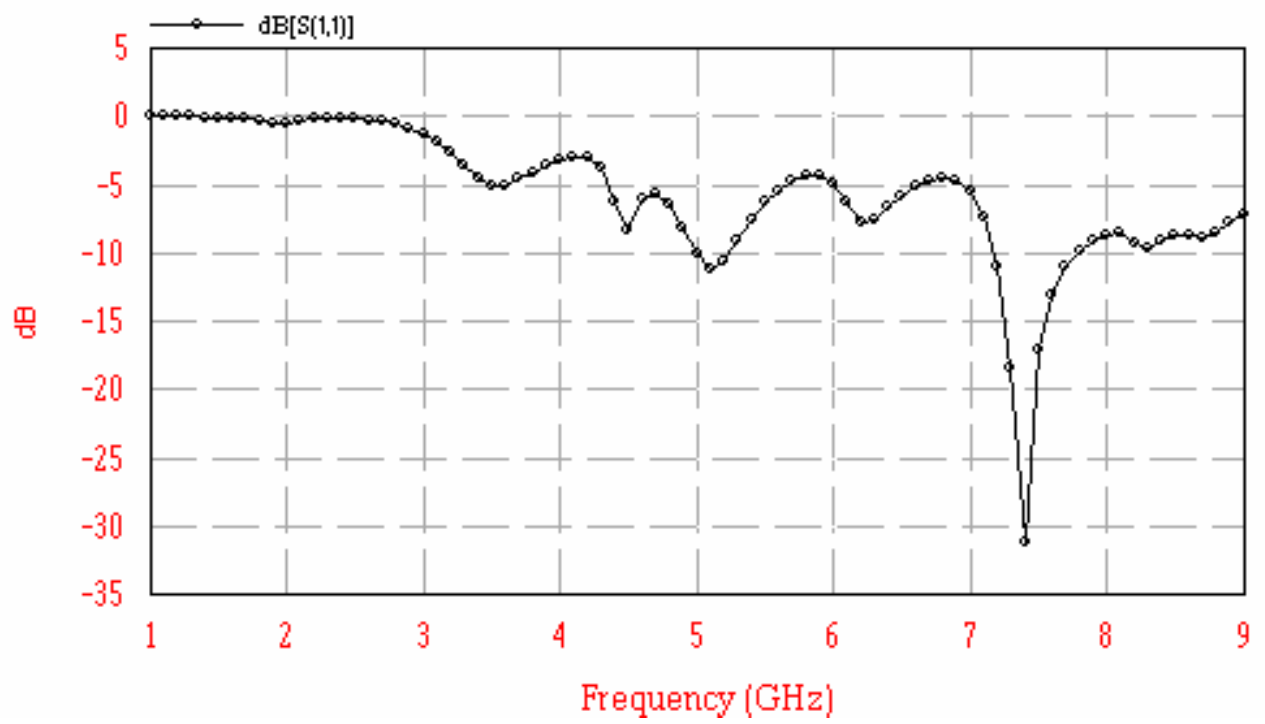

(a)

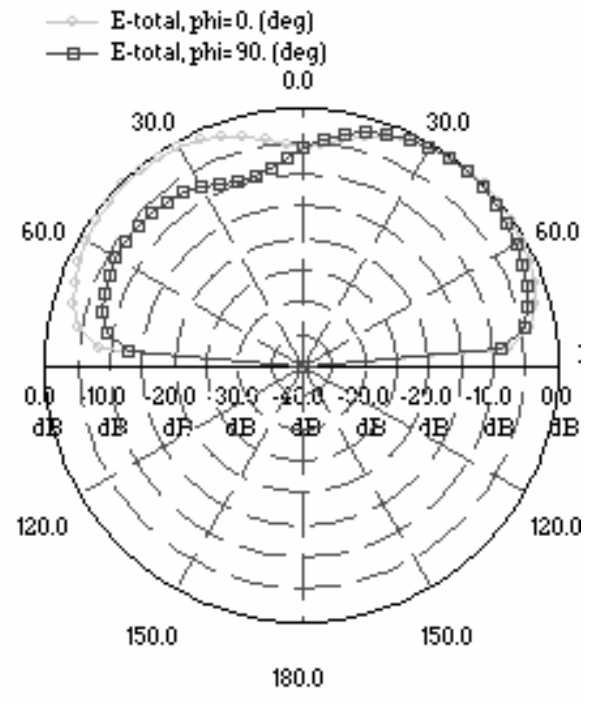

Polar Elevation Pattem

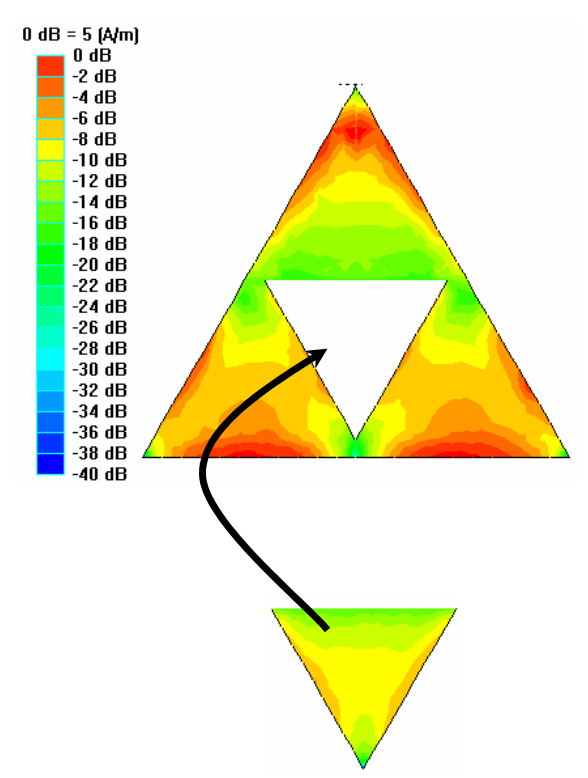

(b) 

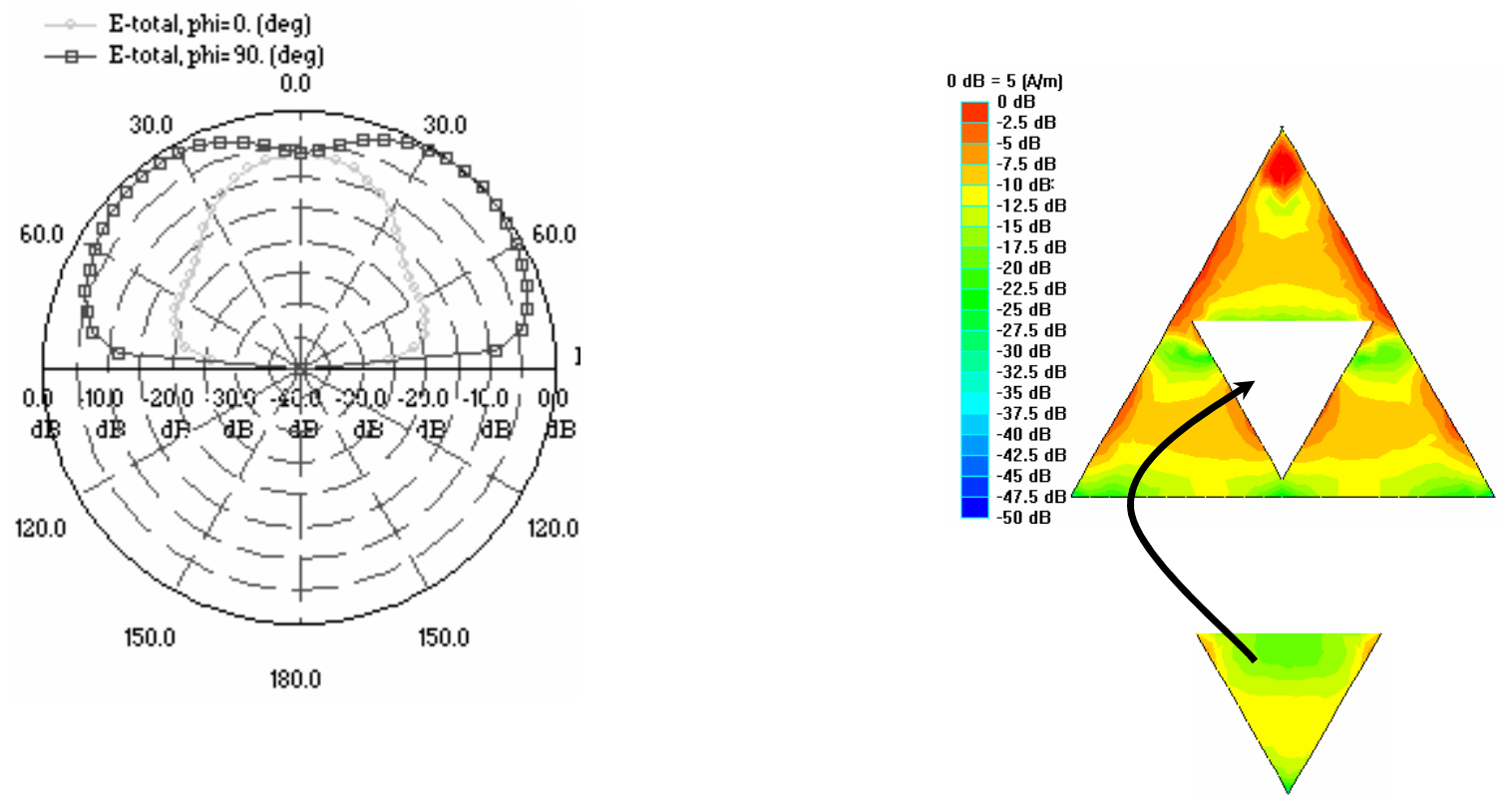

(c)
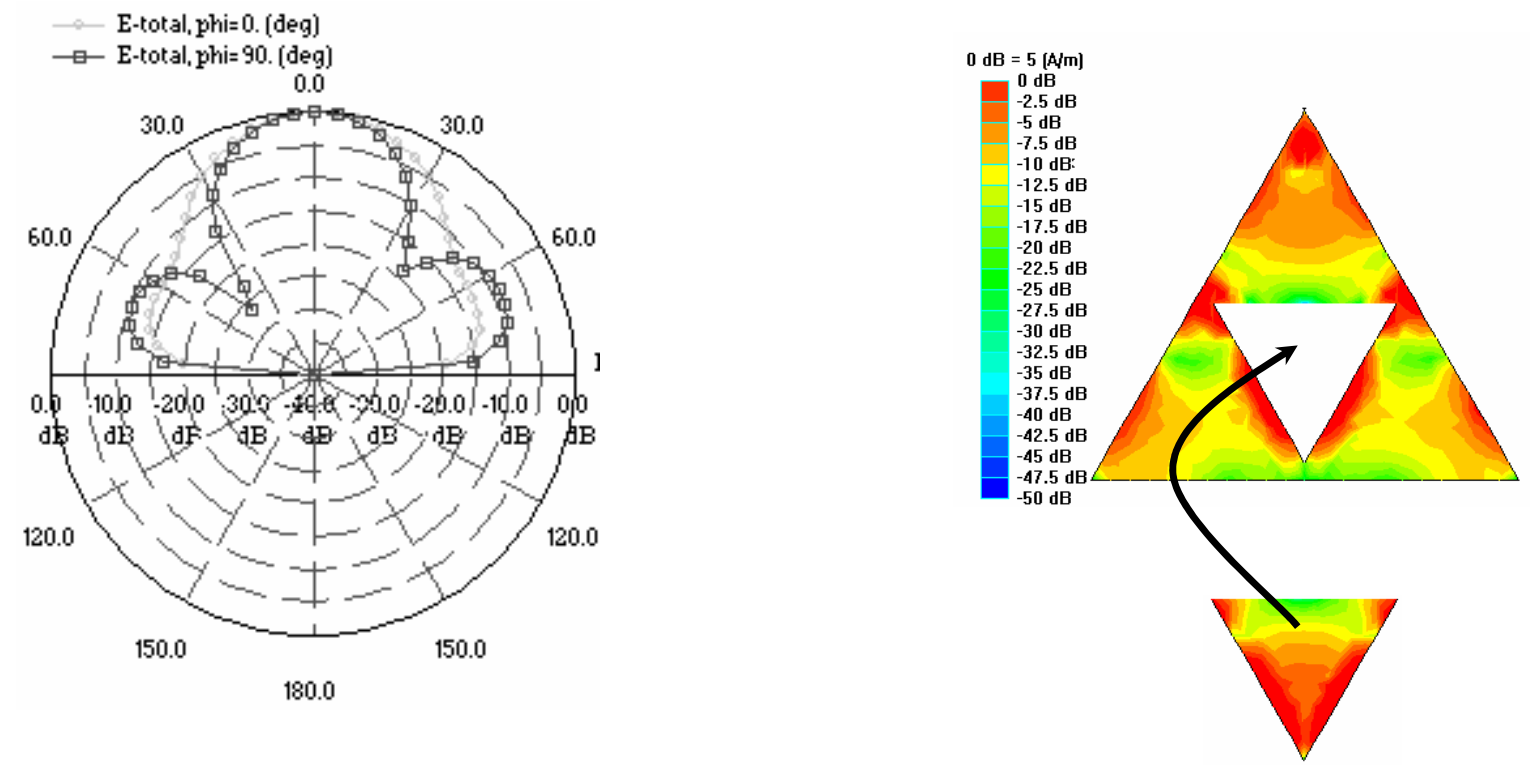

(d) 

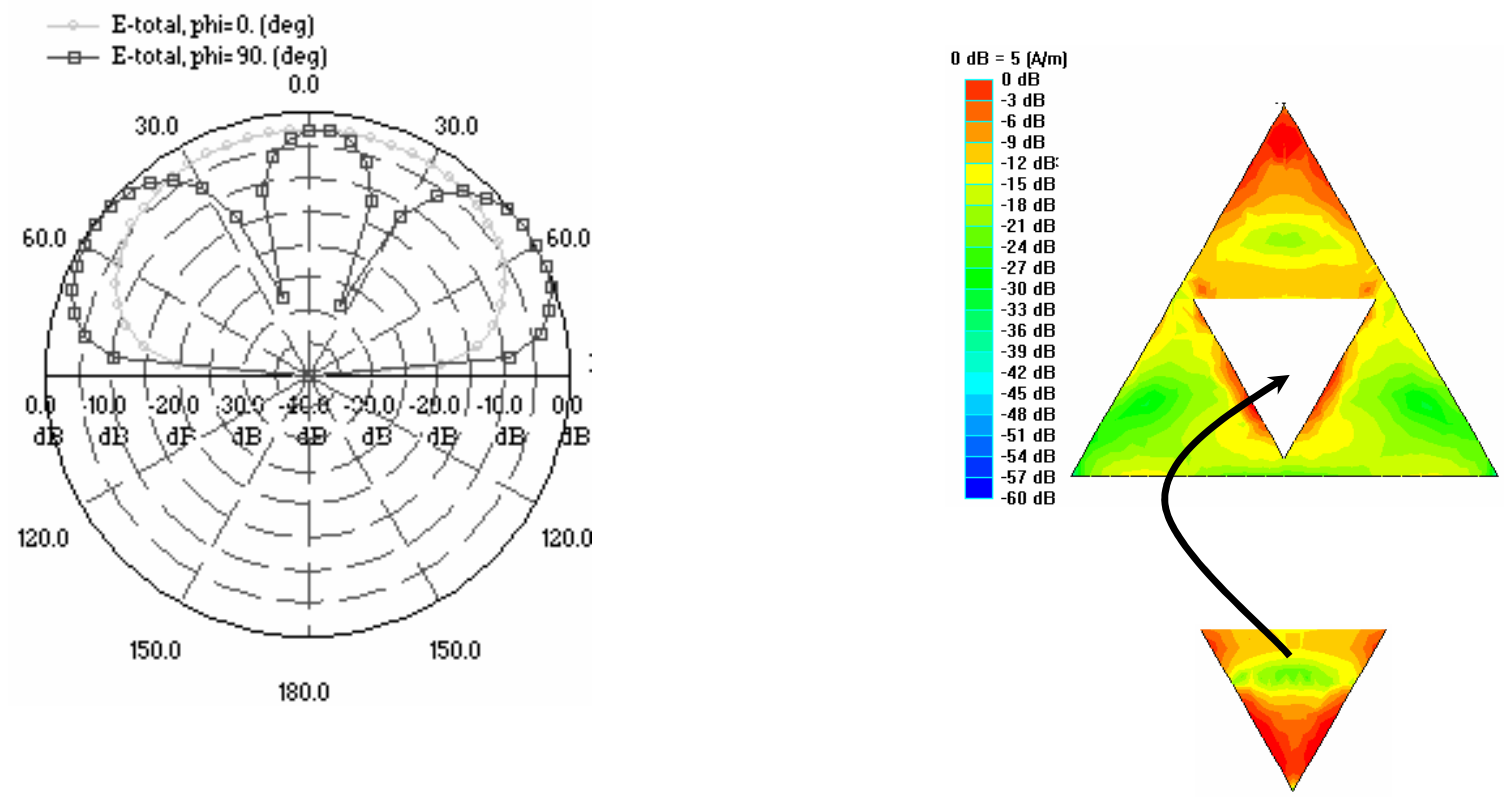

(e)

Figure 24: Characteristics of the Stacked Sierpinski fractal antenna. (a) Return loss, Radiation patterns and current distributions at (b) $4.5 \mathrm{GHz}$, (c) $5.1 \mathrm{GHz}$, (d) 6.2 and (e) $7.4 \mathrm{GHz}$.

\subsection{Effect of truncation}

It was shown that truncation of the corners of the fractal antenna opposite to the apex, where feed is located, can improve the impedance matching [23]. The same principle is applied to the Stacked Sierpinski and Figure 25 shows this antenna with its edges truncated. 


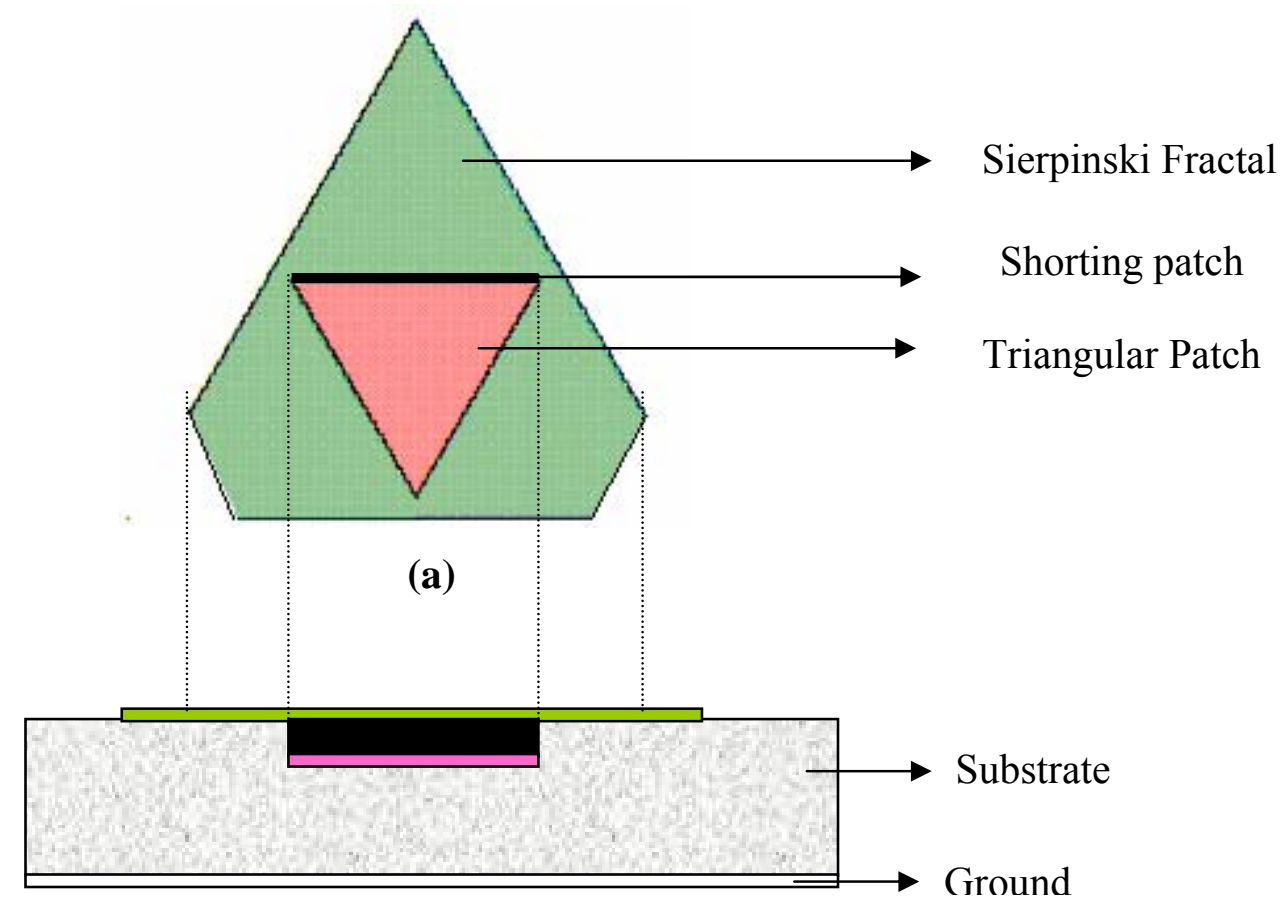

(b)

Figure 25:Layout of truncated stacked Sierpinski antenna (a) top view, and (b) side view.

The input return loss of the truncated antenna is plotted in Figure 26 (a). Four resonances at 4.9, 5.6, 6.5 and $8.6 \mathrm{GHz}$ are seen in the plot. As expected, the impedance matching and hence the return loss for the first three resonances is better than for the untruncated structure shown in Figure 24. Return loss at all three resonances is below $15 \mathrm{~dB}$.

The radiation patterns and current distributions at all the four resonances are shown in (b), (c), (d), (e) of Figure 26. It can be seen that current distribution is altered around the edges due to the truncation, causing the input impedance change and providing a better return loss. 


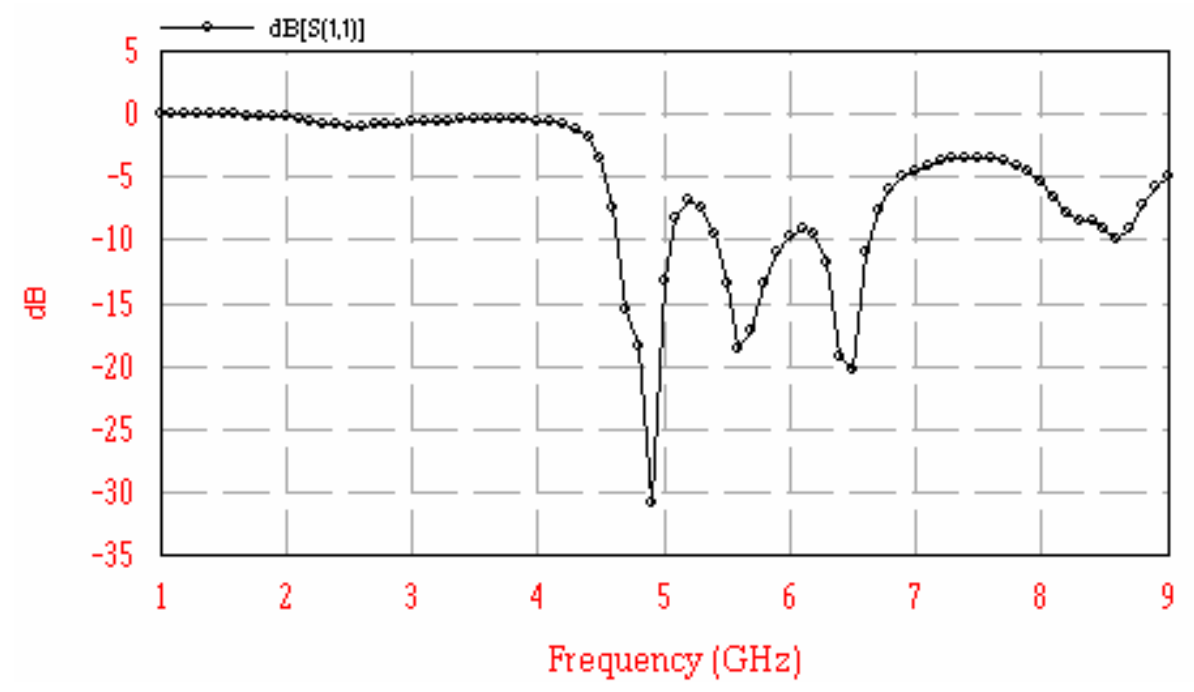

(a)

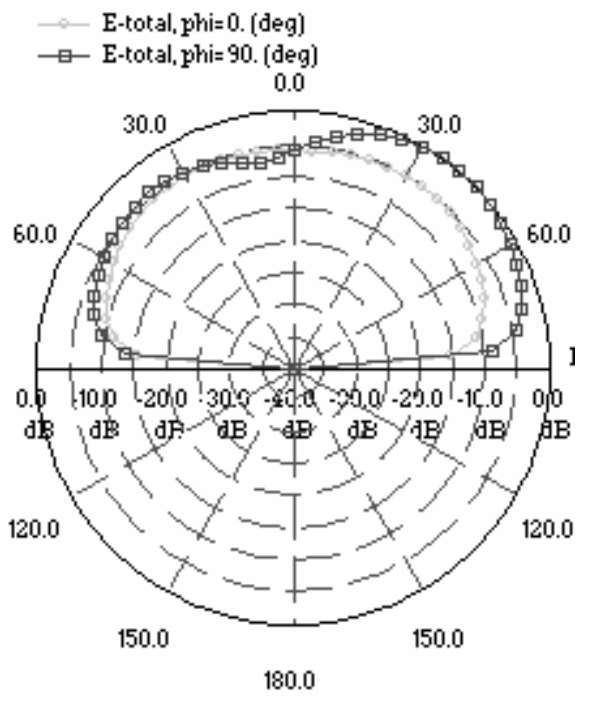

Polar Elevation Pattem

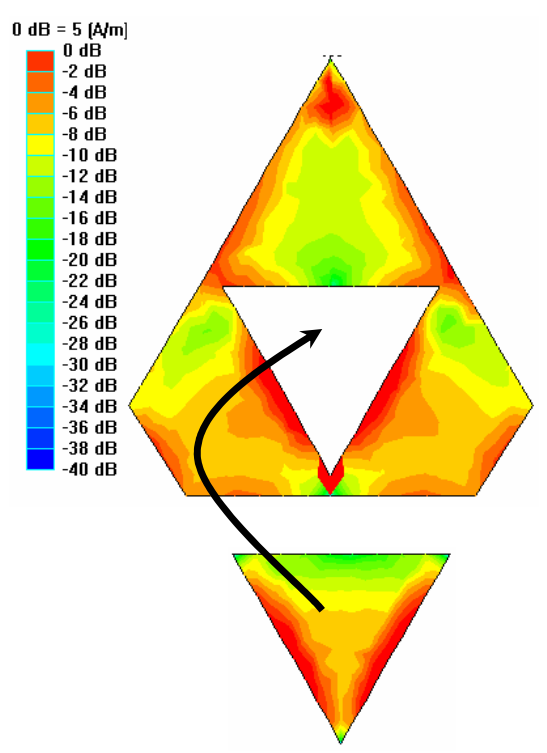

(b) 


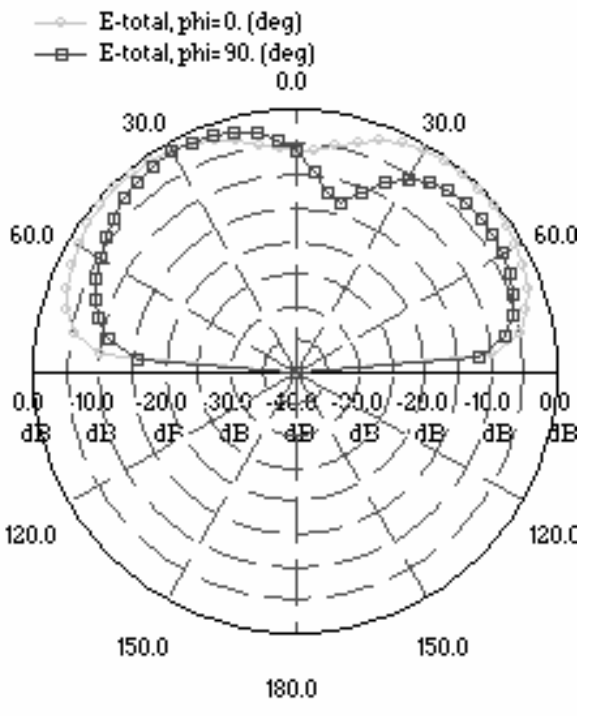

Polar Elevation Pattem

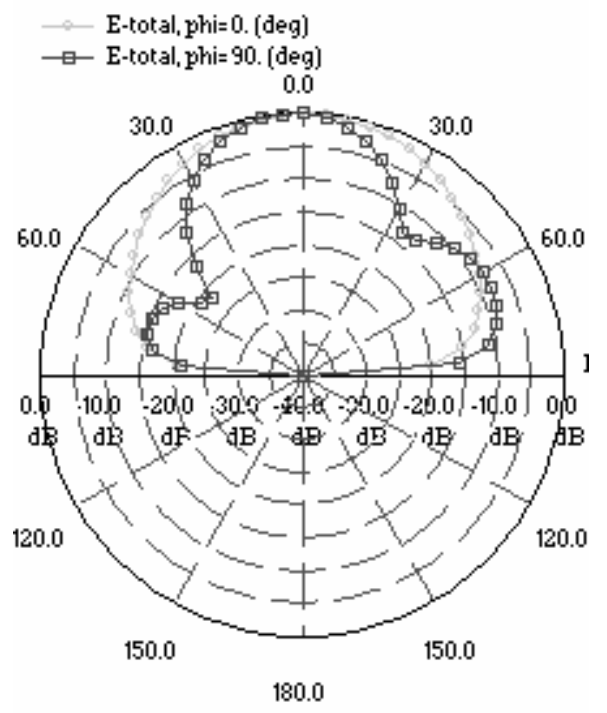

Polar Elevation Pattem

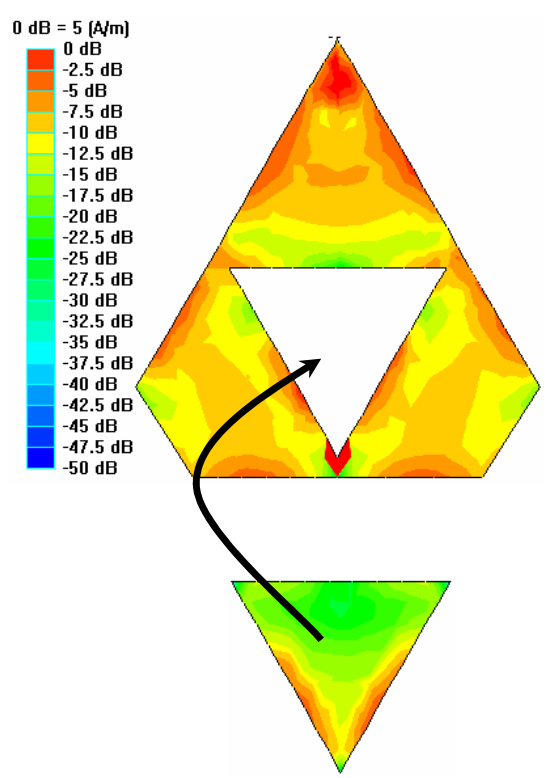

(c)

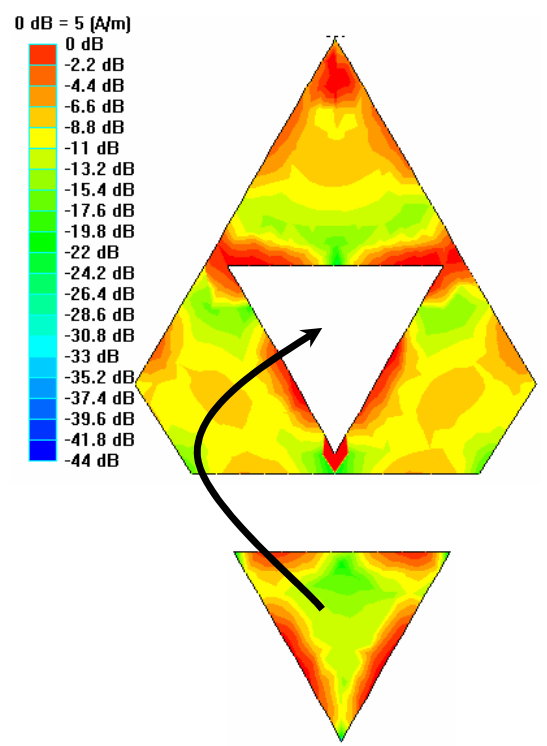

(d) 


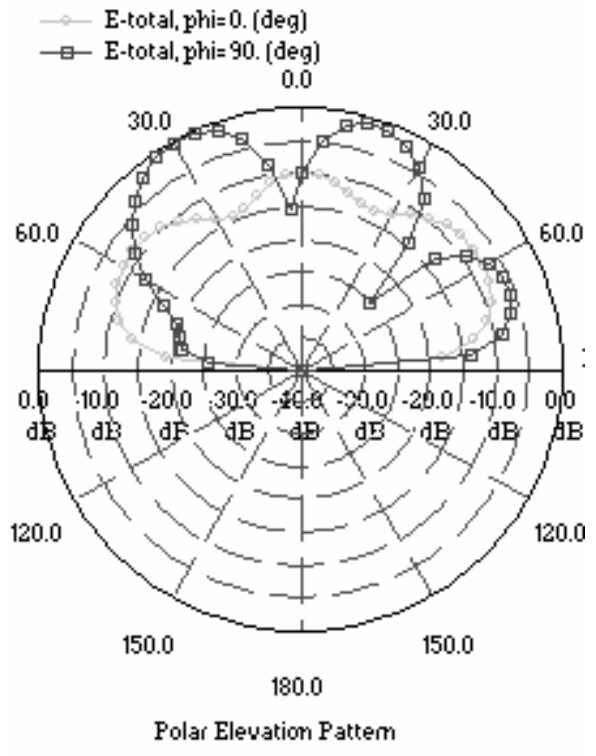
patterns and current distributions at (b) $4.9 \mathrm{GHz}$, (c) $5.6 \mathrm{GHz}$, (d) $6.5 \mathrm{GHz}$ and (e) $8.6 \mathrm{GHz}$

\section{$\underline{\text { 3.6 Gap Coupled Antenna }}$}

The use of a gap at the non-radiating edge, to improve bandwidth of the patch antenna has been analyzed $[25,26]$. An increment of the bandwidth of triangular patch antenna and circular patch antenna using the gap coupled structure without increasing the effective area was analyzed in [25]. In [26], antenna configuration obtained by dividing the rectangular patch into two 45-45-90 triangular patches showed an increased bandwidth. The gap was introduced mainly to improve the bandwidth but it can also provide better return loss at higher frequencies and improved radiation patterns. The results of incorporating this idea in the stacked Sierpinski patch antenna are presented here. 
Figure 27 shows the layout of the gap coupled stacked antenna. The entire antenna, all layers, is split into two halves as shown in the Figure. The gap between the two split halves is $0.5 \mathrm{~mm}$. The structure is feed by coaxial cable on the right half.

(a)

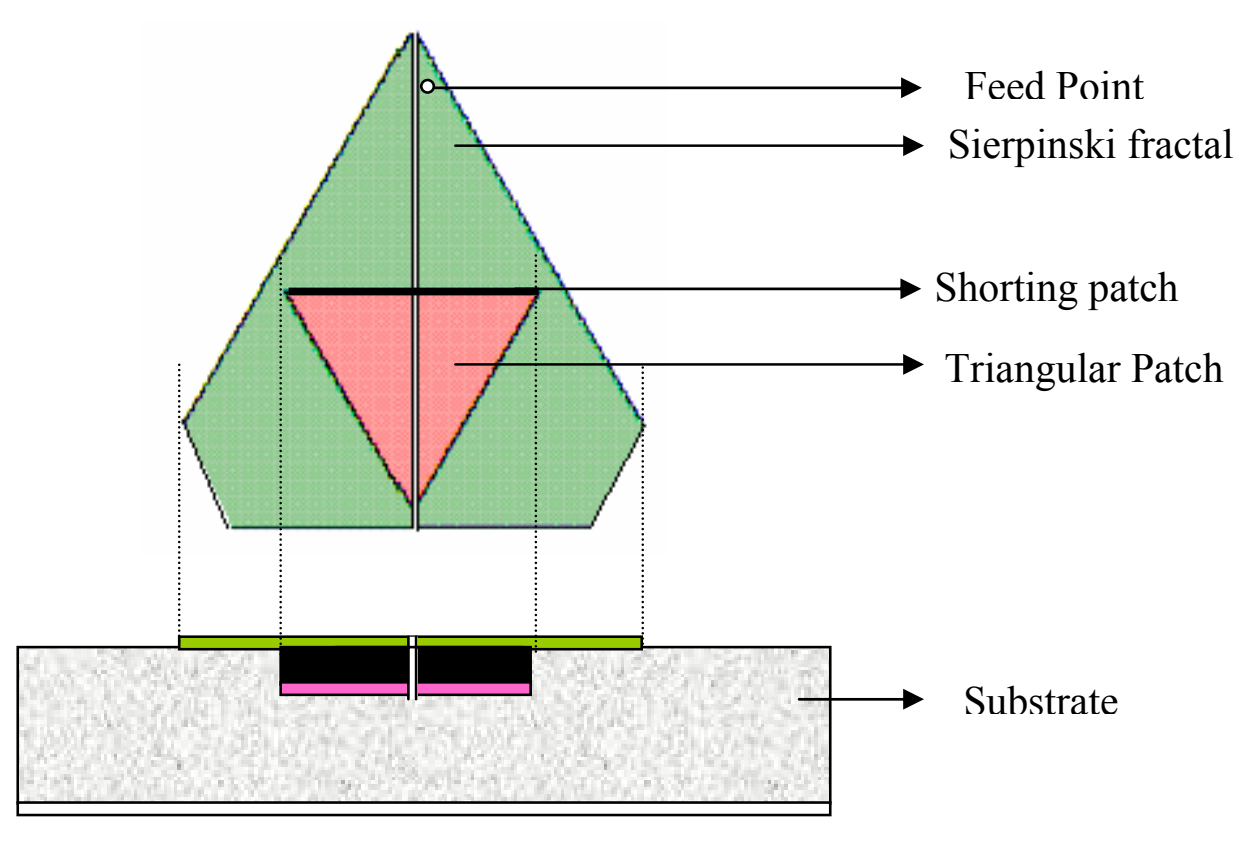

(b)

Figure 27: Layout of the gap coupled antenna. (a) Top view, (b) Side view.

\subsubsection{Simulation result}

Figure 28 shows the result of the gap coupled stacked Sierpinski antenna. A total of six resonances are seen. The first three resonances are the same frequencies as the previous structure. The effect of gap is noticeable at frequencies higher than $7 \mathrm{GHz}$. Three new resonances are now added at $7.2,8$, and $8.7 \mathrm{GHz}$. 
The radiation patterns and current distributions at the resonating frequencies are shown in Figure 28 (b) to (d). Improvement in radiation pattern is seen. In addition, it is seen that the radiation pattern is more omni-directional at all the resonances frequencies.

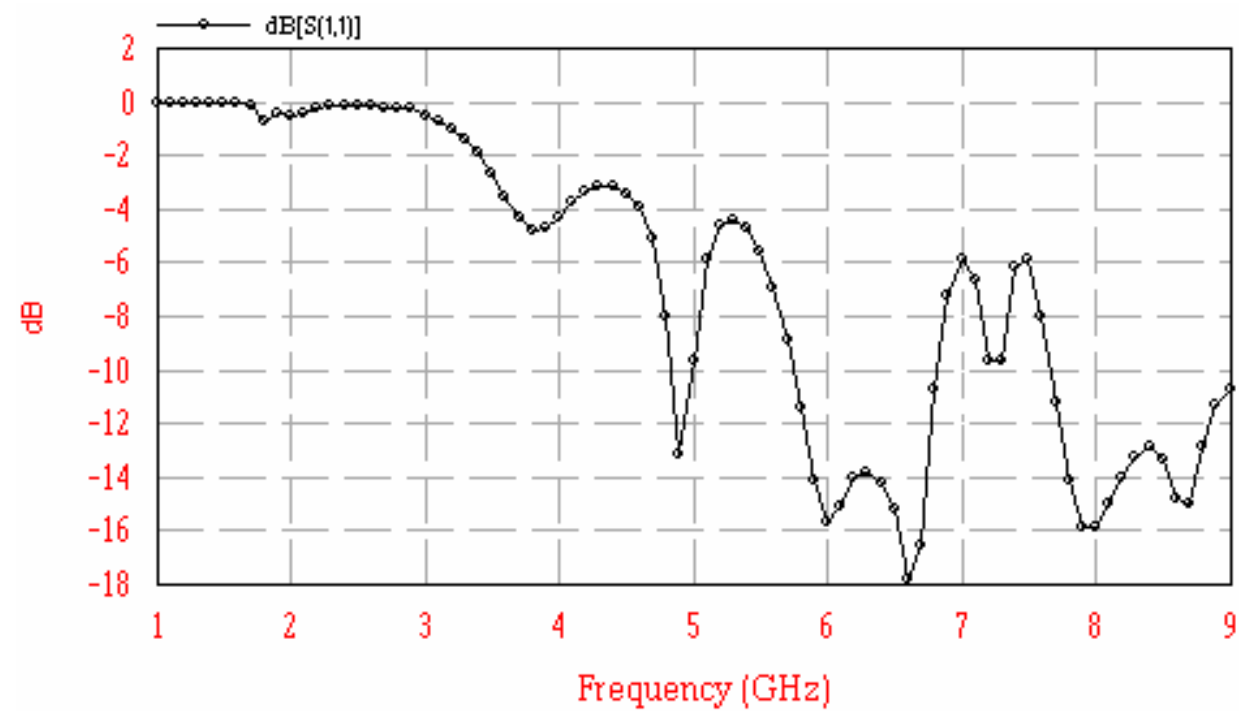

(a)

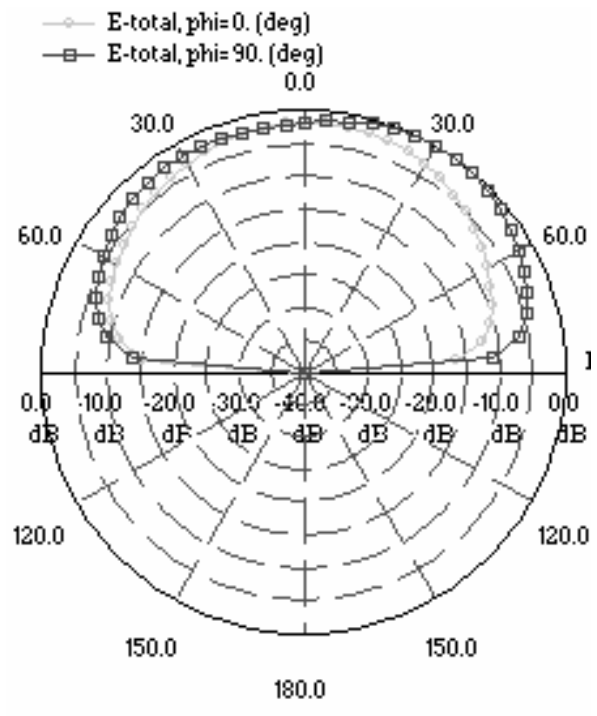

Polar Elevation Pattem

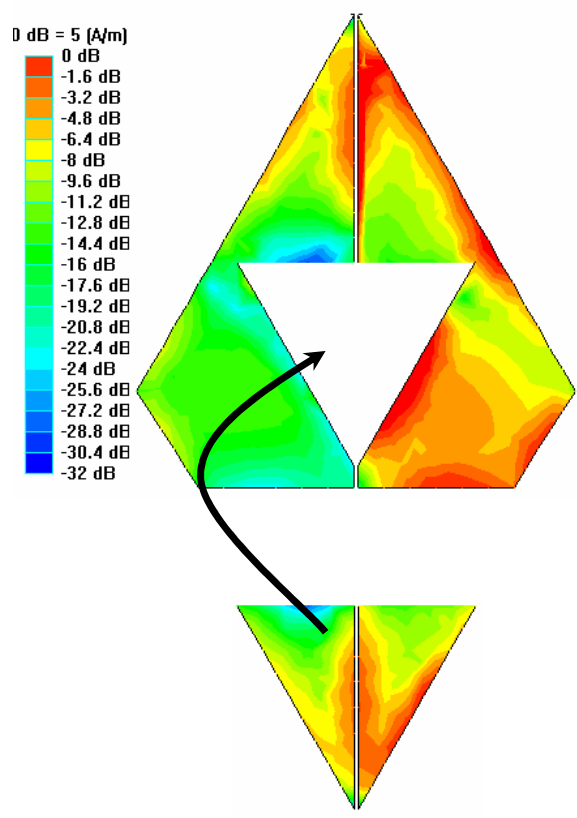

(b) 

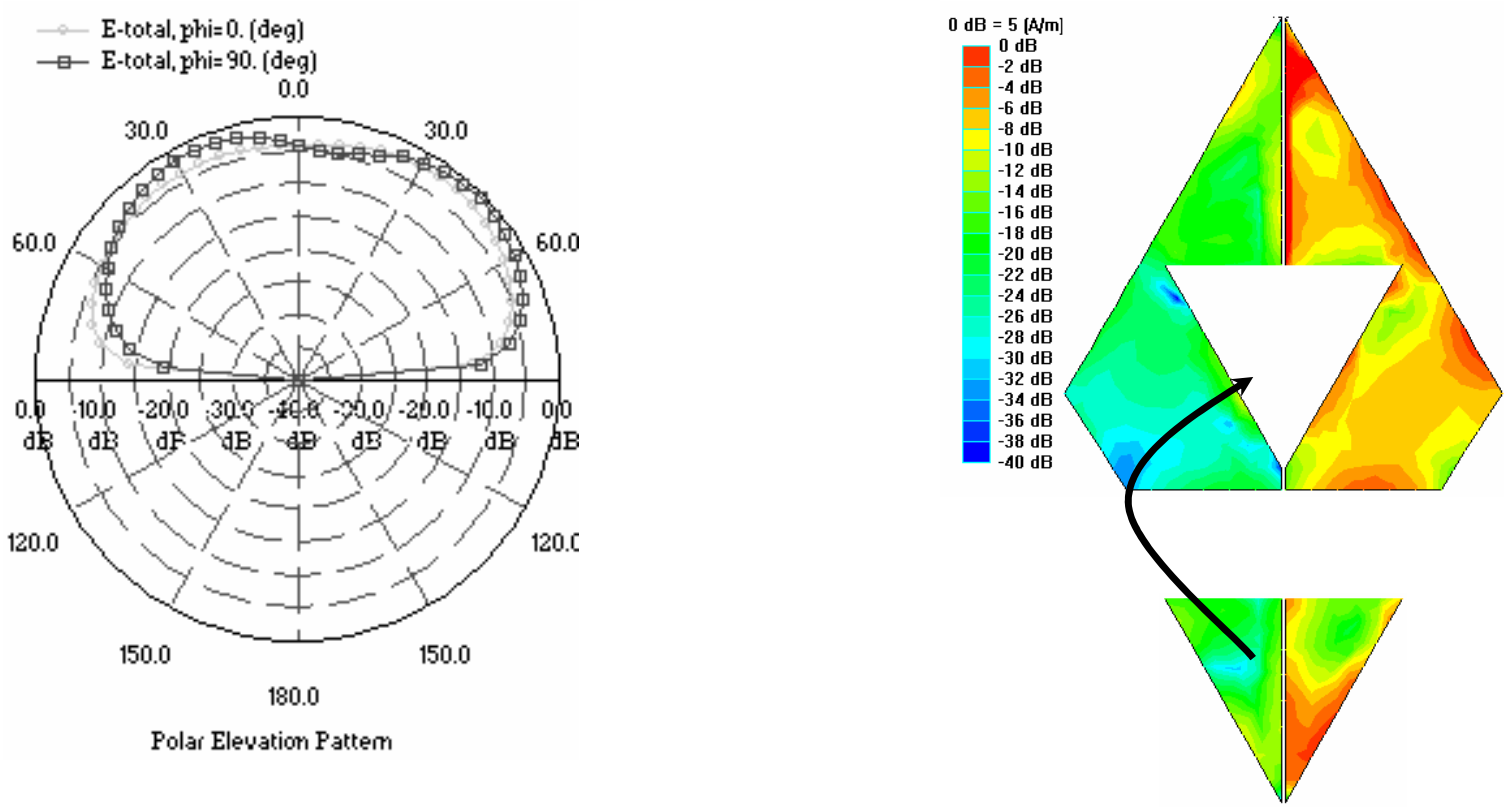

(c)
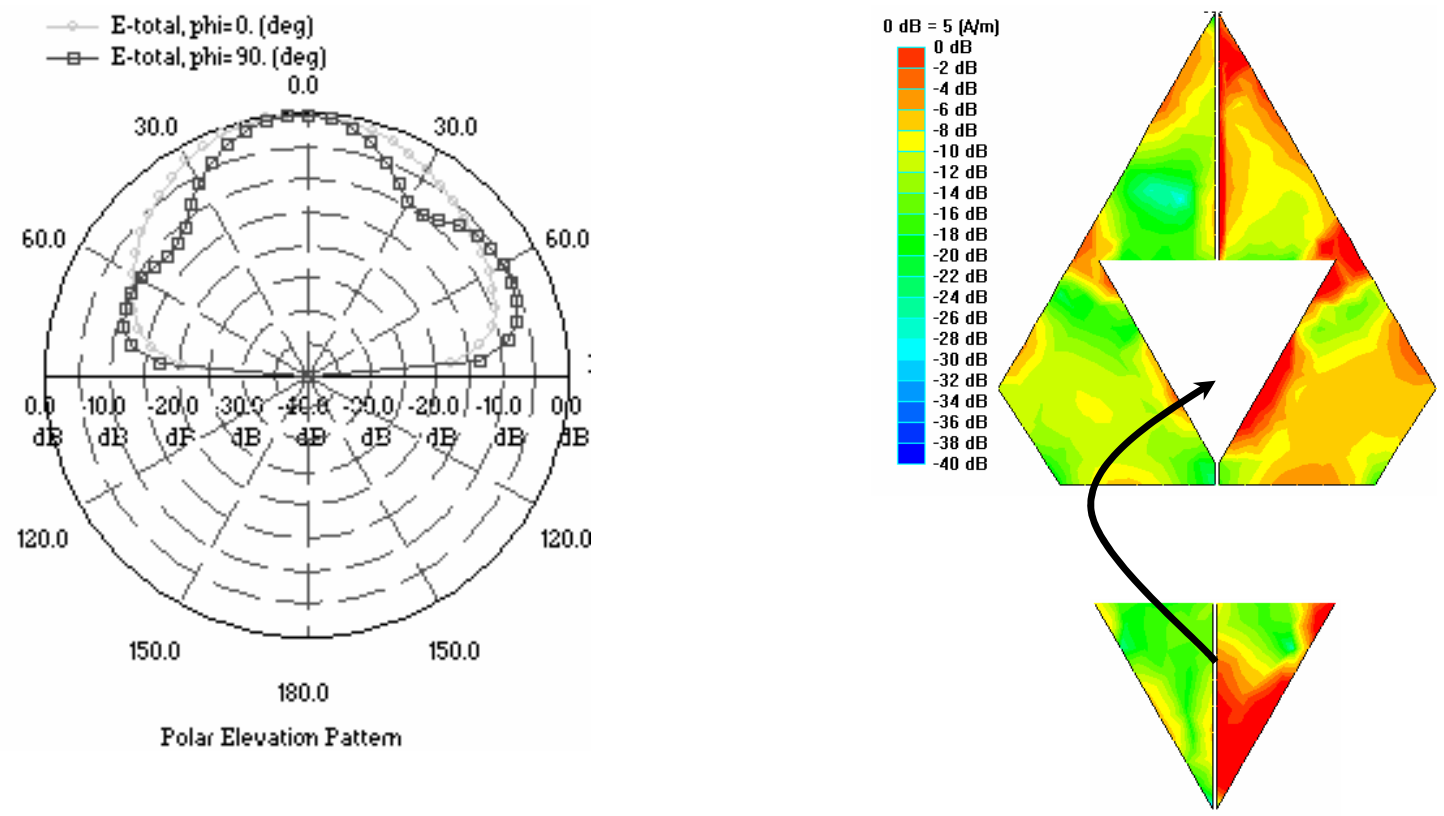

(d) 


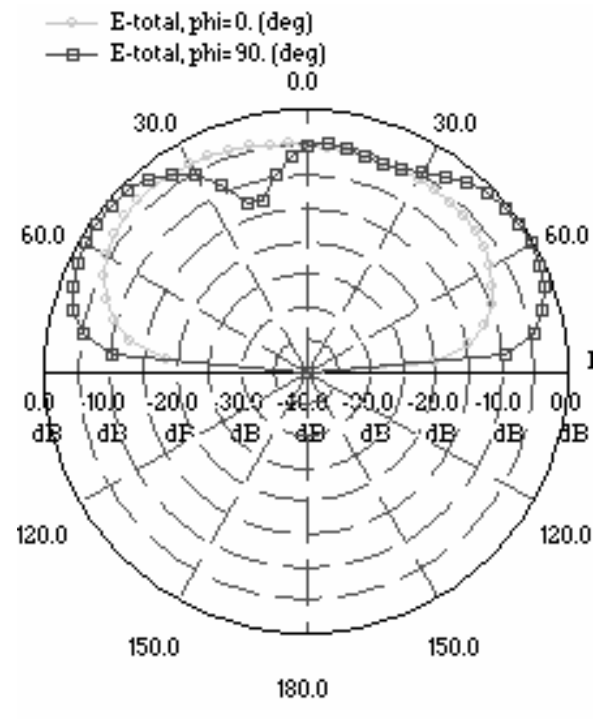

Polar Elevation Pattem

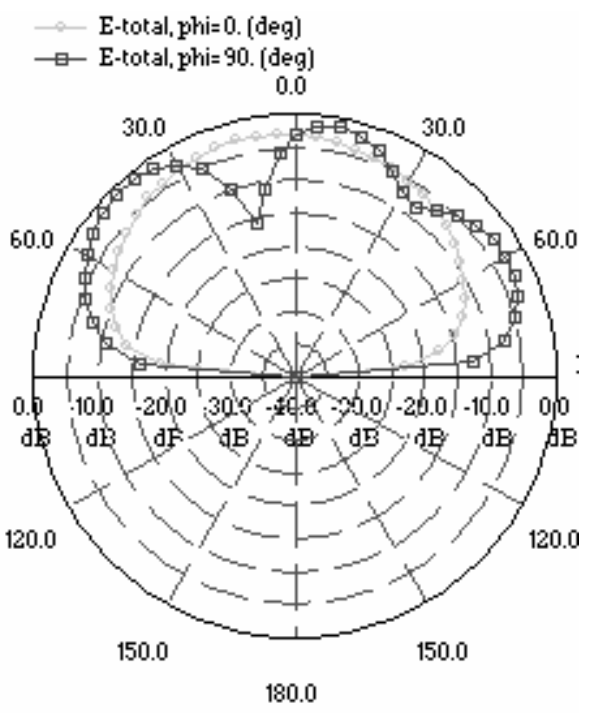

Polar Elevation Pattem

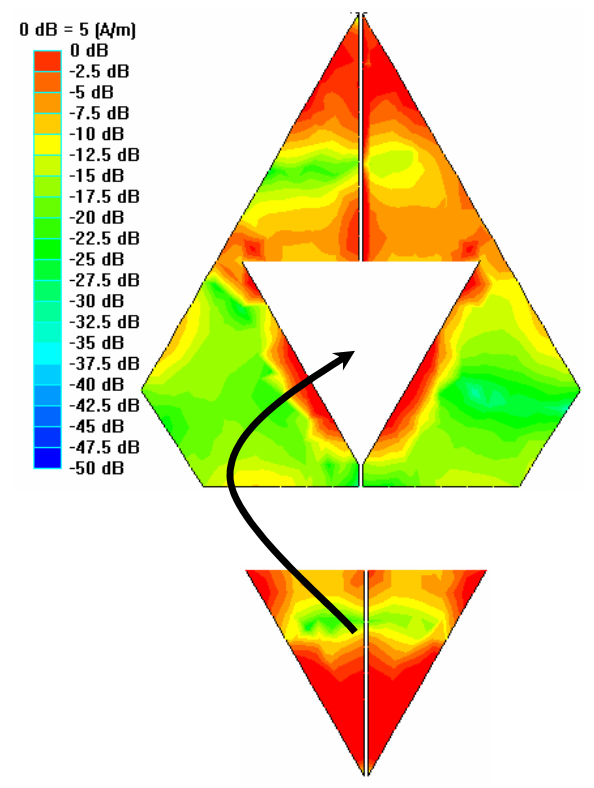

(e)

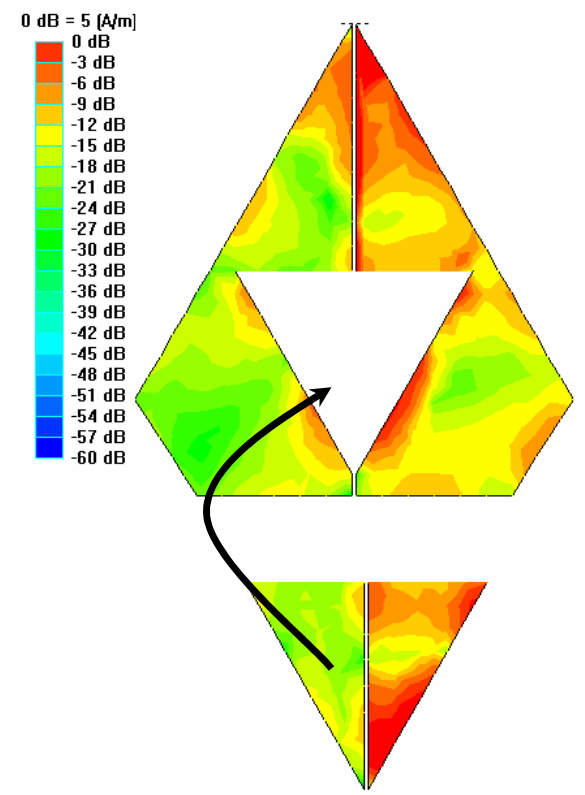



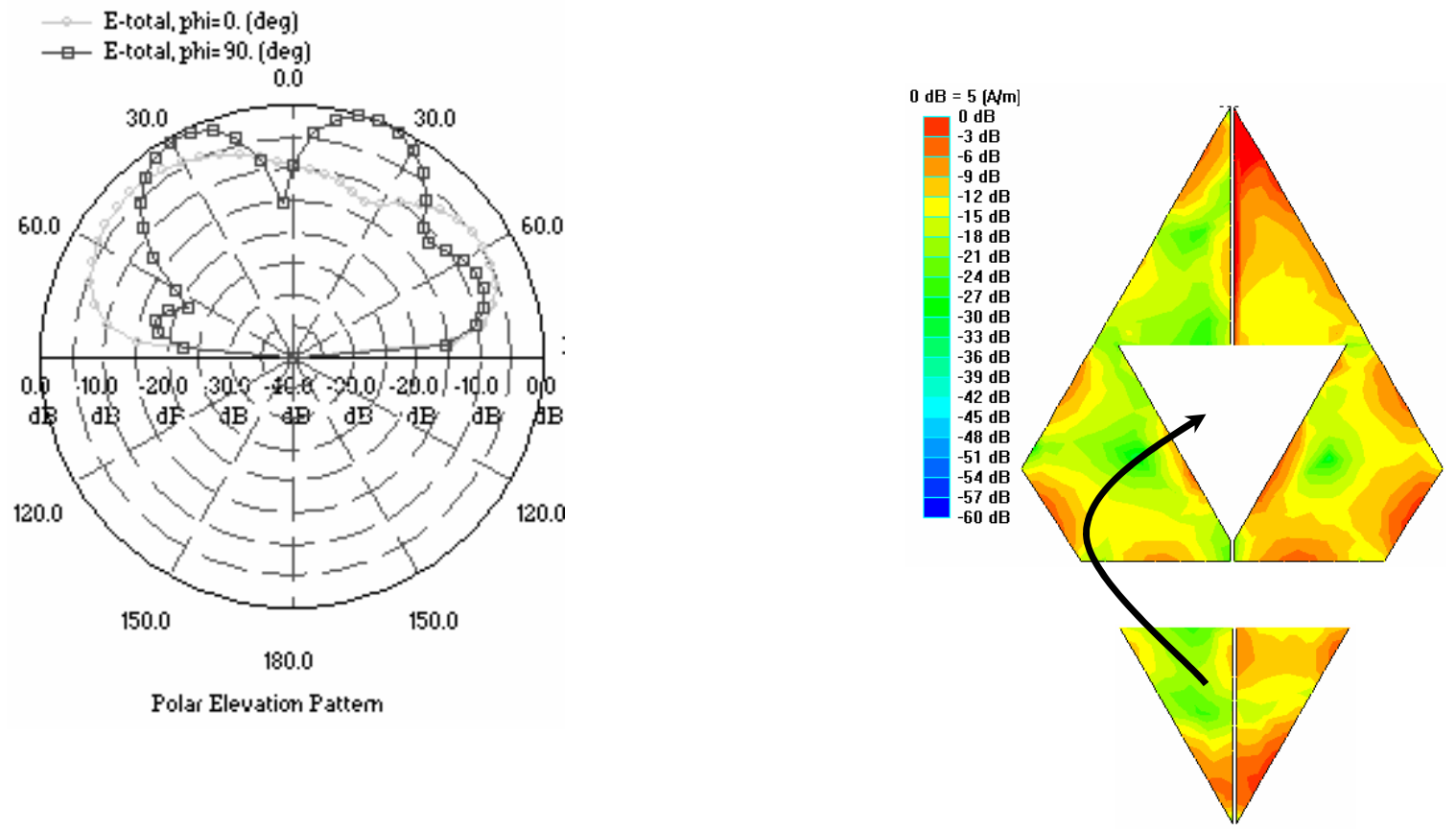

(g)

Figure 28: Characteristics of the gap coupled stacked Sierpinski fractal antenna. (a) Return loss, Radiation patterns and current distributions at (b) $4.9 \mathrm{GHz}$, (c) $6 \mathrm{GHz}$, (d) $6.6 \mathrm{GHz}$, (e) $7.2 \mathrm{GHz}$, (f) $8 \mathrm{GHz}$, and (g) $8.7 \mathrm{GHz}$. 


\section{CHAPTER 4: CONCLUSION AND FUTURE RESEARCH}

A schematic approach to understand the multi-band properties of Sierpinski fractal monopole antenna were done based on published literature. It was shown that the Sierpinski fractal patch antenna doesn't posses the same multi-band property due to lack of coupling between the triangular patches. Several steps taken to make the Sierpinski patch antenna behave as a multi-band antenna are analyzed.

A modified stacked Sierpinski patch antenna obtained by merging the top grid layer with the fractal antenna is presented. This resulted in an additional resonance but has a very poor return loss. Truncating the corner of the Sierpinski structure helped to improve the return loss. A gap structure was analyzed next to obtain an increase in bandwidth. The gap is introduced along the non- radiating edge of the antenna. The effect of the gap is more noticeable at higher frequencies. This also produced patterns that were more omni-directional. The proposed designs provide more parameters allowing one to control the resonances and the patterns. The work could be extended to third and forth order fractals. 


\section{LIST OF REFERENCES}

1. H.O. Peitgen, H. Jurgens, D. Saupe, Chaos and Fractals: New Frontiers of Science, Springer, New York, 1992.

2. M. Dekking, J. L. Vehel, E. Lutton, and C. Tricot (editors), Fractals: Theory and Application in Engineering, Springer-Verlag, London, 1999, pp. 125-151.

3. J. L. Vehel, E. Lutton, and C. Tricot (editors), Fractals in Enginnering, Springe- Verlag, London, 1997, pp. 222-236.

4. D. L. Jaggard and Y. Kim, “ Diffraction by Band-limited Fractal Screens”, Journal of the Optical Society of America, vol.4, no.6, June 1987, pp. 1055-1062.

5. J. Giamvittorio, and Y. Rahmat- Samii, “Fractal FSS: Various Self-Similar Geometries Used for Dual Band and Dual polarized FSS', IEEE Antennas and Propagation Society International Symposium, July 2001.

6. C. Puente, J. Romeu, R. Pous, J. Ramis, and A. HIjazo, "Small but Long Koch Fractal Monopole”, IEEE Electronics Letters, vol. 34, pp 9-10, January 1998.

7. C. Puente Balirda, J. Romeu, A. Cardama, "The Koch Monopole: A small Fractal Antenna", IEEE Transactions on Antenna and Propagation, vol.48, No.11, November 2000, pp.1773-1781.

8. J. Romeu, C.Borja, S. Blanch, "High Directivity Modes in the Koch Island Fractal Patch Antenna", IEEE Antennas and Propagation Society International Symposium, July 2000. 
9. C. Puente, J. Romeu, R. Pous, A. Cardama, "On the behavior of the Sierpinski Multiband Fractal Antenna”, IEEE Transactions on Antenna and Propagation, vol.46, no. 4, pp.517524, April 1998.

10. J. Solar, and J. Romeu, "Dual band Sierpinski Fractal Monopole Antenna", IEEE Antenna and Propagation Society International Symposium, July 2000.

11. C. Borja, and J. Romeu, "Multiband Sierpinski Fractal Patch Antenna", IEEE Antenna and Propagation Society International Symposium, July 2000.

12. C. Puente, J. Romeu, R. Pous, X. Garcia, and F. Benitez, “Fractal Multiband Antenna based on the Sierpinski gasket", Electronic Letters, vol.32, no.1, $4^{\text {th }}$ January 1996, pp.1-2.

13. D.H. Werner, R. L. Haupt, and P. L. Werner, "Fractal Antenna Engineering: The Theory and Design of Fractal Antenna Arrays", IEEE Antenans and Propagation Magazine, vol.41, No. 5, October 1999, pp.37-59.

14. D. H. Werner, and P. L Werner, "On the Synthsis of Fractal Radiation Patterns", Radio Science, vol. 30, no. 1, January- February 1995, pp. 29-45.

15. D. Baldacci, D.H Werner, "An Efficient Recursive Procedure for Calculating the Driving Point Impedance of Linear and Planar fractal Arrays", IEEE Antennas and Propagation Society International Symposium, July 2001.

16. C. Puente, M. Navarro, J. Romeu, R. Pous, "Variations on the Fractal Sierpinski Antenna Flare Angle", IEEE Antennas and Propagation Society International Symposium, July 1998.

17. C.T.P Song, P.S. Hall, H. Ghafouri-Shiraz, and I. Henning, "Fractal Antenna Research at University of Birmingham", IEEE $11^{\text {th }}$ International Conference on Antennas and Propagation, 17-20 April 2001. 
18. S.R. Best, "The Sierpinski Gasket: Modified Non-Fractal Gap Structures Exhibiting Multi-Band Behavior”, IEEE Antennas and Propagation Society International Symposium, 2002.

19. D. Anagnostou, M Khodier, J.C. Lyke, and C.G. Chiristodoulou, "Re-configurable Sierpinski Gasket Antenna using RF- MEMS Switches”, IEEE Antennas and Propagation Society International Symposium, 2003.

20. D. Anagnostou, M Khodier, J.C. Lyke, and C.G. Chiristodoulou, "Fractal Antenna with RF MEMS Switches for Multiple Frequency Applications”, IEEE Antennas and Propagation Society International Symposium, 2002

21. J. Yeo and R. Mitttra, "Modified Sierpinski Gasket Patch Antenna for Multiband Applications”, IEEE Antennas and Propagation Society International Symposium, 2001.

22. C.T.P Song, P.S. Hall, H. Ghafouri-Shiraz, and I. Henning, "Shorted Fractal Sierpinski Monopole Antenna”, IEEE Antennas and Propagation Society International Symposium, 2001.

23. J. Yeo and R. Mitttra, “A Novel Modified Sierpinski Patch Antenna using Shorting Pins and Switches for Multiband Applications", IEEE Antennas and Propagation Society International Symposium, 2002.

24. R. Garg, P. Bhartia, I. Bahl, A. Ittipiboon, "Microstrip Antenna Design Handbook”, Artech House, 2001.

25. M.B. Nile, A.A. Rasheed, G. Kumar, "Broadband Gap Coupled Semicircular and Triangular Microstrip Antennas”, IEEE Antennas and Propagation Society International Symposium, 1994. 
26. D.C. Chang and J. Zheng, "A Wide-Band Microstrip Antenna Using Two Triangular Patches”, IEEE Antennas and Propagation Society International Symposium, 1991. 